Universidade de São Paulo

Instituto de Física

\title{
Unitariedade em Teorias Não Comutativas
}

\author{
Pedro Rogério Sérgi Gomes \\ ORIENTADOR: Prof. Dr. Marcelo Otavio Caminha Gomes
}

Dissertação de Mestrado apresentada ao Instituto de Física para obtenção do título de Mestre em Ciências

Comissão Examinadora

Prof. Dr. Marcelo Otavio Caminha Gomes (IFUSP)

Prof. Dr. Fernando Tadeu Caldeira Brandt (IFUSP)

Prof. Dr. Adriano Antonio Natale (IFT)

São Paulo

2009 
Theoretical Physics is truly blessed, in that the quests for truth and beauty coincide. Kerson Huang 


\section{Agradecimentos}

Registro aqui o nome das pessoas a quem desejo verdadeiramente referir meus agradecimentos. O Professor Marcelo Otavio Caminha Gomes, pelos ensinamentos, paciência e por toda atenção ao longo deste trabalho. Minha namorada, Paula Fernanda Bienzobaz, por ser a pessoa maravilhosa que é, sempre ao meu lado. Meus pais, Pedro Waldir Gomes e Maria Aparecida Sérgi Gomes e minha irmã Maria Carolina Sérgi Gomes, por nossa relação harmoniosa, formando uma família tão especial. O Professor Renio dos Santos Mendes, pessoa e físico admirável. Leandro Ibiapina Beviláqua e Renann Lipinski Jusinskas, companheiros do CFT307. O CNPq pela bolsa concedida. Tomo a liberdade aqui de expressar minha profunda admiração pelo grande físico teórico Lev Davidovich Landau (1908-1968), o qual muito me inspira. 


\section{Resumo}

Este trabalho é dedicado ao estudo da unitariedade em teoria de campos não comutativa. Inicialmente, são apresentadas as ferramentas básicas para abordar o problema da unitariedade em teorias não comutativas, incluindo as regras de corte e uma introdução à não comutatividade. Em seguida, foi feita a análise do modelo $\phi^{3}$ não comutativo. Empregando o esquema perturbativo usual da teoria de campos, foi verificado que o modelo é unitário quando a não comutatividade envolve apenas o espaço. Por outro lado, quando a não comutatividade envolve o espaço e o tempo verificou-se uma violação da unitariedade, fato esse bem conhecido tratando-se de teorias não comutativas. Partimos então para uma abordagem proposta na literatura, em que a teoria de perturbação é adaptada para teorias não comutativas. Dentro desse esquema, o modelo $\phi^{3}$ foi estudado novamente verificando assim a unitariedade para um diagrama de um laço e segunda ordem na constante de acoplamento mesmo quando a não comutatividade envolve o espaço e o tempo. Baseado nesse método, estendemos a análise para uma teoria contendo além de um campo escalar um campo fermiônico, mais precisamente o modelo de Yukawa, no qual também foi verificada a unitariedade a um laço e segunda ordem na constante de acoplamento. 


\section{Abstract}

This work is dedicated to study unitarity in noncommutative field theory. Initially, the basic tools to handle the problem of unitarity in noncommutative theories are discussed, including the cutting rules and an introduction to noncommutativity. Then, we analised the noncommutative $\phi^{3}$ model. Using the usual perturbative framework of field theory, we verified that the model preserves unitarity when the noncommutativity is restrict to the spatial coordinates. On the other hand, when the noncommutativity includes both, space and time, we found a violation of the unitarity, a well known fact in noncommutative field theory. Next, we turn to an approach proposed in the literature, in wich perturbation theory is adapted for noncommutative field theory. Whitin this approach, the $\phi^{3}$ model was studied again and the unitarity was verified for one loop diagram and second order in the coupling constant even in the case when noncommutativity affects both space and time. Following this, we extended the analysis to a field theory with fermionic and scalar

fields, namely, Yukawa's model, again verifing unitarity at one loop and second order in the coupling constant. 


\section{Sumário}

$\begin{array}{lr}\text { Introdução } & 8\end{array}$

1 Regras de Corte e Não Comutatividade $\quad 11$

1.1 Unitariedade e Regras de Corte . . . . . . . . . . . . . . . . . . . . 11

1.1.1 Propagador e algumas propriedades . . . . . . . . . . . . . . 12

$1.1 .2 \quad$ Regras de Corte . . . . . . . . . . . . . . . . . . . . . 13

1.2 Não Comutatividade . . . . . . . . . . . . . . . . . . . . . . . . . . . 21

2 Unitariedade no modelo $\phi^{3}$ não comutativo 28

2.1 Modelo $\phi^{3}$ Não Comutativo . . . . . . . . . . . . . . . . . . . . 28

2.1 .1 Diagrama de um laço . . . . . . . . . . . . . . . . . . . 31

2.1 .2 Não comutatividade espacial: $p \circ p>0 \ldots \ldots \ldots \ldots \ldots$

2.1 .3 Diagrama cortado . . . . . . . . . . . . . . . 37

2.1.4 Não comutatividade espaço-temporal: $p \circ p<0 \ldots \ldots$

3 Perturbação para Teorias Não Comutativas 42

3.1 Algumas Definições . . . . . . . . . . . . . . . . . . . . . . . . . . . . 42

3.2 Campos Assintóticos . . . . . . . . . . . . . . . . . . . . . . . 43

3.3 Matriz $S \ldots \ldots \ldots \ldots \ldots \ldots \ldots \ldots \ldots$

3.4 Matriz $S$ e Campos $\phi_{i n}$ e $\phi_{e m} \ldots \ldots \ldots \ldots \ldots$

3.5 Unitariedade . . . . . . . . . . . . . . . . . . . . . . . . 54

4 Modelo $\phi^{3}$ Não Comutativo $\quad 57$

4.1 Unitariedade até um laço . . . . . . . . . . . . . . . . . . . . 57

5 Modelo de Yukawa $\quad 68$

5.1 Diagrama com pernas externas fermiônicas . . . . . . . . . . . . 68

5.2 Diagrama com pernas externas bosônicas . . . . . . . . . . . . . . . . 79

Considerações Finais e Perspectivas $\quad 85$

$\begin{array}{ll}\text { A Propagador anti-ordenado temporalmente } & 87\end{array}$ 
$\begin{array}{ll}\text { B Produto Moyal envolvendo campos iguais } & 89\end{array}$

$\begin{array}{ll}\text { C Férmions de Majorana } & 93\end{array}$

D Produto Moyal com campos fermiônicos de Majorana 95

E Demonstração de que os produtos matriciais em (5.32) são reais $\quad 97$

F Demonstração da igualdade $A_{1}^{\dagger}=A_{1}$ para o modelo de Yukawa $\quad 100$

G Produto Moyal com prescrição minimal $\quad 101$

H Cálculo de unitariedade no modelo de Yukawa 103

$\begin{array}{ll}\text { Referências Bibliográficas } & 106\end{array}$ 


\section{Introdução}

O problema das divergências ultravioletas presentes nas séries perturbativas da teoria quântica dos campos está relacionado com o produto de campos no mesmo ponto que, em princípio, não está bem definido. Esse fato pode ser visto na própria regra de quantização canônica: $[\phi(\mathbf{x}, t), \pi(\mathbf{y}, t)] \equiv i \delta^{3}(\mathbf{x}-\mathbf{y})$, visto que a função delta não é bem definida quando $\mathbf{x}=\mathbf{y}$. Reside nesse fato uma das origens da teoria de campos não comutativa [1]. Inicialmente, foi pensado que a introdução de operadores de posição que não comutam, eliminando assim a idéia de ponto $\left(\Delta x^{\mu} \Delta x^{\nu} \sim \Theta^{\mu \nu}\right)$, pudesse resolver o problema dos infinitos. Com o aparecimento do procedimento de renormalização para tratar tais divergências, as idéias de se trabalhar com teorias não comutativas foram postas de lado.

Recentemente, o interesse por teorias não comutativas reapareceu com grande ímpeto no contexto da teoria de cordas, principalmente, devido à descoberta de estruturas típicas de teorias não comutativas na dinâmica de uma corda aberta na presença de um campo magnético de fundo [2]. Além dessa, tratando-se de gravitação existe o argumento heurístico pelo qual, em distâncias da ordem da distância de Planck, $10^{-33} \mathrm{~cm}$, medidas de coordenadas não fazem mais sentido, visto que o aparecimento de um campo gravitacional intenso impede a luz ou outro sinal de transmitir informação [3]. Nesse sentido, o conceito de ponto deve ser eliminado conduzindo assim à idéia de operadores de posição que não comutam.

Uma série de novos fenômenos surgem quando a não comutatividade é implementada. Dentre eles destacamos a mistura ultravioleta/infravermelho [4], que consiste na transmutação de parte das divergências ultravioletas do caso comutativo em singularidades infravermelhas que, em certas condições, podem quebrar os esquemas perturbativos usuais. Além desse, destacamos o problema da violação da unitariedade. Como mostrado por Gomis e Mehen [5], a aplicação direta de métodos perturbativos usados no caso comutativo, porém com regras de Feynman modificadas, leva a uma quebra de unitariedade quando a não comutatividade envolve o espaço e o tempo. A propósito, um dos capítulos desta dissertação trata-se da reprodução desse resultado. Diversos aspectos de não comutatividade podem ser encontrados nos artigos de revisão citados nas referências [6] e $[7]$.

Diferentes propostas surgiram na literatura na tentativa de solucionar os problemas 
mencionados. No que segue, citamos algumas relacionadas principalmente à unitariedade e esboçamos suas idéias. Nos trabalhos [8] e [9] de Liao e Sibold, a abordagem é feita a partir das funções de Green, levando em conta que o produto Moyal (devido às derivadas temporais) não comuta com o ordenamento temporal, sendo justamente esse o problema do tratamento em [5]. Assim, a unitariedade é preservada em teorias de campos escalares. Entretanto, quando essa análise é estendida para o caso de teorias de gauge, verifica-se a violação de indentidades de Ward [10], mostrando que o problema da unitariedade não pode ser resolvido com esse método. Na referência [11] é mostrado que a violação da unitariedade em teorias com não comutatividade espaço-temporal se dá devido à definição imprópria da teoria quântica de campos sobre espaços não comutativos. As idéias desenvolvidas em [11] foram posteriormente elaboradas por Rim e Yee [12] e por Rim, Seo e Yee [13]. Essas duas últimas referências também compõe parte desta dissertação como será melhor explicado. A vantagem do método proposto em [12] e [13] é que não há problemas com teorias de gauge [14]. Uma comparação entre os métodos e resultados das referências [8][9] e [12][13], do ponto de vista de integração funcional, é feita em [15] que, além disso, inclui outros aspectos de teorias não locais no tempo.

Este trabalho tem como principal objetivo o estudo sobre a unitariedade. No que segue, passemos a descrever a estrutura do texto. No primeiro capítulo são apresentadas as ferramentas básicas para abordar o problema da unitariedade em teorias não comutativas. Elas são constituídas das regras de corte [16][17], que permitem uma análise sistemática da unitariedade, seguida de uma introdução à não comutatividade em teoria de campos [19][20], em que são discutidos diversas propriedades da mesma. No segundo capítulo, partimos para o estudo do modelo $\phi^{3}$ seguindo o trabalho [5]. Essencialmente, são efetuados cálculos para verificação de uma relação de unitariedade entre diagramas obtida a partir das regras de corte estabelecidas no primeiro capítulo. Devido aos resultados obtidos, mais precisamente uma violação da relação de unitariedade no caso em que o espaço e o tempo são envolvidos pela não comutatividade, uma proposta diferente se faz necessária. Portanto, no terceiro capítulo, apresentamos um método perturbativo para teorias escalares não comutativas de acordo com o artigo listado em [13]. Nele, soluções iterativas da equação de Klein-Gordon são consideradas. Também, definimos uma matriz $S$, a qual o ordenamento temporal é tratado por meio da realização mininal. Como a matriz $S$ permite-nos obter uma relação entre os campos assintóticos (que são soluções da equação de Klein-Gordon homogênea), podemos compará-los com aqueles obtidos via soluções iterativas, como é feito no texto. O resultado é que, além da matriz $S$ conectar corretamente os campos assintóticos, a unitariedade se preserva. O passo seguinte é considerar modelos específicos. Assim, empregando esse método retornamos ao modelo $\phi^{3}$ no capítulo quatro. De acordo com a condição de unitariedade para a matriz $S$, verificamos que a unitariedade, a um laço, é preservada independentemente se a não comutatividade envolve o espaço e o tempo ou apenas o espaço. Esse estudo foi baseado no artigo [12]. No último capítulo, estendemos a análise anterior para o caso de uma teoria envolvendo campos fermiônicos. Mais precisamente, investigamos a unitariedade para um diagrama 
de um laço no modelo de Yukawa, cuja lagrangiana de interação envolve um campo escalar e dois campos fermiônicos. O resultado obtido para o diagrama estudado foi que a unitariedade também é preservada.

Por fim, vamos à definição das notações usadas ao longo do trabalho. O sistema de unidades natural é empregado, tal que $c=\hbar=1$. A métrica é dada por $g^{\mu \nu}=$ $\operatorname{diag}(1,-1,-1,-1)$. Índices gregos variam de 0 a $D-1$ e índices latinos variam de 1 a $D-1$, sendo $D$ a dimensão do espaço-tempo. Também usamos a convenção de soma, em que índices repetidos estão somados. Além disso, as fórmulas mais comumente usadas em teoria de campos são apresentadas sem a demonstração, como o propagador escalar, as formas explícitas dos campos escalar e fermiônico, entre outras. Elas seguem a notação utilizada em [21] e [22], as quais também servem como referêncial geral. As fórmulas matemáticas ao longo do texto, principalmente do segundo capítulo, foram extraídas de $[23]$. 


\section{Capítulo 1}

\section{Regras de Corte e Não Comutatividade}

Este capítulo é dedicado ao estudo das regras de corte, que podem ser usadas para investigar o problema da unitariedade em teoria de campos. Além disso, apresentamos uma breve introdução à teoria quântica de campos não comutativa. Esses tópicos são ferramentas básicas para abordar o problema da unitariedade em alguns modelos de teorias não comutativas, que é o objetivo principal deste trabalho.

\subsection{Unitariedade e Regras de Corte}

A unitariedade da matriz $S$ significa

$$
S^{\dagger} S=S S^{\dagger}=1 .
$$

Essa condição é um dos requerimentos fundamentais para a interpretação quântica de uma teoria, visto que ela implica na conservação de probabilidades associadas aos estados inicial e final de um dado processo. Separando a parte devido a interação, podemos escrever a matriz $S$ como

$$
S=1+i T .
$$

Em termos da matriz $T$, a condição (1.1) torna-se

$$
-i\left(T-T^{\dagger}\right)=T T^{\dagger} .
$$

Tomando o elemento de matriz entre os estados $|a\rangle$ e $|b\rangle$ e introduzindo do lado direito um conjunto completo de estados, obtemos 


$$
-i\left\langle b\left|\left(T-T^{\dagger}\right)\right| a\right\rangle=\sum_{c}\langle b|T| c\rangle\left\langle c\left|T^{\dagger}\right| a\right\rangle .
$$

Se $T_{a b}=T_{b a}$, o lado esquerdo é essencialmente a parte imaginária da amplitude $T_{a b}$. Lembramos que $T_{a b}$ é dado por uma soma de diagramas de Feynman que contribuem para um certo processo. Nesse sentido é útil conhecer a parte imaginária de um diagrama de Feynman. Esse é um objetivo das regras de corte (ou de Cutkosky). Essas regras permitem-nos calcular a parte imaginária de um diagrama de Feynman de uma maneira sistemática, conduzindo a uma conexão com a unitariedade da teoria.

\subsubsection{Propagador e algumas propriedades}

Antes de iniciar propriamente o estudo das regras de corte, precisamos fazer uma análise sobre as origens das partes imaginárias que eventualmente aparecem numa dada amplitude. Primeiramente, os vértices são definidos a partir de $i \mathcal{L}_{\text {int }}$, sendo $\mathcal{L}_{\text {int }}$ a densidade de lagrangiana de interação da teoria. Se a interação for hermitiana, o vértice é puramente imaginário. A outra possibilidade vem do fator de $i$ da definição do propagador e da prescrição $i \epsilon$ para tratar seu pólo. Como mencionado, esses fatores são responsáveis pelas partes imaginárias dos diagramas.

Além das regras de Feynman usuais, o próximo passo é escrever regras de Feynman para o complexo conjugado dos vértices e do propagador, pois assim poderemos obter representações diagramáticas para o complexo conjugado de um gráfico qualquer. Nesse sentido, veremos algumas quantidades relevantes do campo escalar. O propagador do campo escalar com massa $m$, em quatro dimensões, é dado por

$$
\Delta_{F}(x)=\int \frac{d^{4} p}{(2 \pi)^{4}} \frac{i}{p^{2}-m^{2}+i \epsilon} e^{-i p x} .
$$

Ele pode ser decomposto em partes com frequência positiva e negativa, $\Delta^{+}$e $\Delta^{-}$, da seguinte maneira,

$$
\Delta_{F}(x)=\theta\left(x^{0}\right) \Delta^{+}(x)+\theta\left(-x^{0}\right) \Delta^{-}(x),
$$

tal que

$$
\Delta^{ \pm}(x)=\int \frac{d^{4} p}{(2 \pi)^{4}} 2 \pi \theta\left( \pm p^{0}\right) \delta\left(p^{2}-m^{2}\right) e^{-i p x} .
$$

Notemos que os propagadores $\Delta^{ \pm}$estão sobre a concha de massa, ao passo que o propagador de Feynman $\Delta_{F}$ não. Além disso, eles satisfazem as seguintes propriedades:

$$
\Delta^{ \pm}(-x)=\Delta^{\mp}(x)=\left[\Delta^{ \pm}(x)\right]^{*}
$$


e

$$
\Delta_{F}(-x)=\Delta_{F}(x) .
$$

Podemos definir o propagador anti-ordenado temporalmente, $\bar{\Delta}$, por meio de

$$
\bar{\Delta}(x)=\theta\left(x^{0}\right) \Delta^{-}(x)+\theta\left(-x^{0}\right) \Delta^{+}(x) .
$$

A partir dessa relação é interessante obter uma expressão para $\bar{\Delta}$ análoga à (1.5). Esse cálculo é mostrado no apêndice A. O resultado obtido é

$$
\bar{\Delta}(x)=\int \frac{d^{4} p}{(2 \pi)^{4}} \frac{-i}{p^{2}-m^{2}-i \epsilon} e^{-i p x} .
$$

Notemos que, como deveria ser, o propagador anti-ordenado é justamente o complexo conjugado de (1.5), ou seja,

$$
\bar{\Delta}(x)=\left(\Delta_{F}(x)\right)^{*} .
$$

Outra observação importante é que nem todas as funções definidas acima são independentes, pois

$$
\begin{aligned}
\Delta_{F}(x)+\bar{\Delta}(x) & =\left[\theta\left(x^{0}\right)+\theta\left(-x^{0}\right)\right] \Delta^{+}(x)+\left[\theta\left(-x^{0}\right)+\theta\left(x^{0}\right)\right] \Delta^{-}(x) \\
& =\Delta^{+}(x)+\Delta^{-}(x) .
\end{aligned}
$$

Após essa breve discussão de algumas propriedades das funções relativas ao campo escalar, partiremos para as regras de Feynman e as regras de corte.

\subsubsection{Regras de Corte}

Dada uma teoria, podemos definir regras de Feynman associadas a ela. Qualquer amplitude será então constituída de vértices e propagadores de acordo com as regras estabelecidas. No espaço coordenado, o integrando dessa amplitude será escrito como uma função das coordenadas dos vértices. Para discutir essa questão com mais detalhes, consideremos a teoria escalar com interação $\phi^{3}$. As regras de Feynman dessa teoria são mostradas na figura (1.1). A figura (1.2) mostra um exemplo de uma amplitude construída a partir das regras de Feynman.

O passo seguinte é construir outras representações diagramáticas para incluir o complexo conjugado dos propagadores e dos vértices. Essas regras de Feynman adicionais juntamente com as regras usuais são mostradas na figura (1.3). 


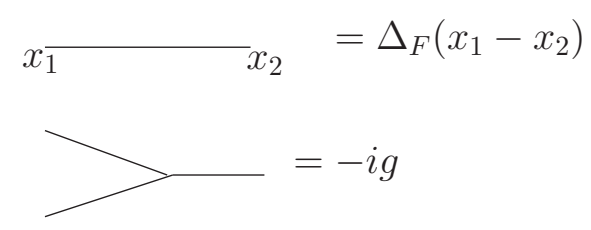

Figura 1.1: Regras de Feynman de $\phi^{3}$.

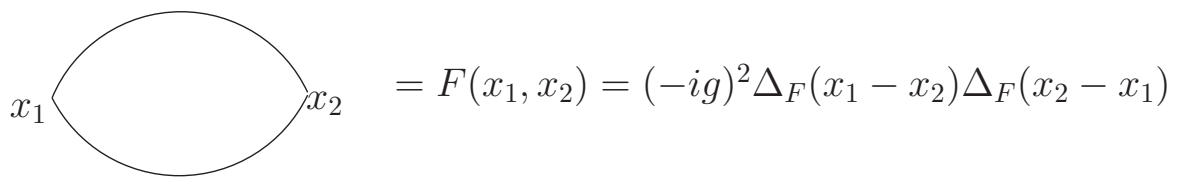

Figura 1.2: Exemplo de amplitude.

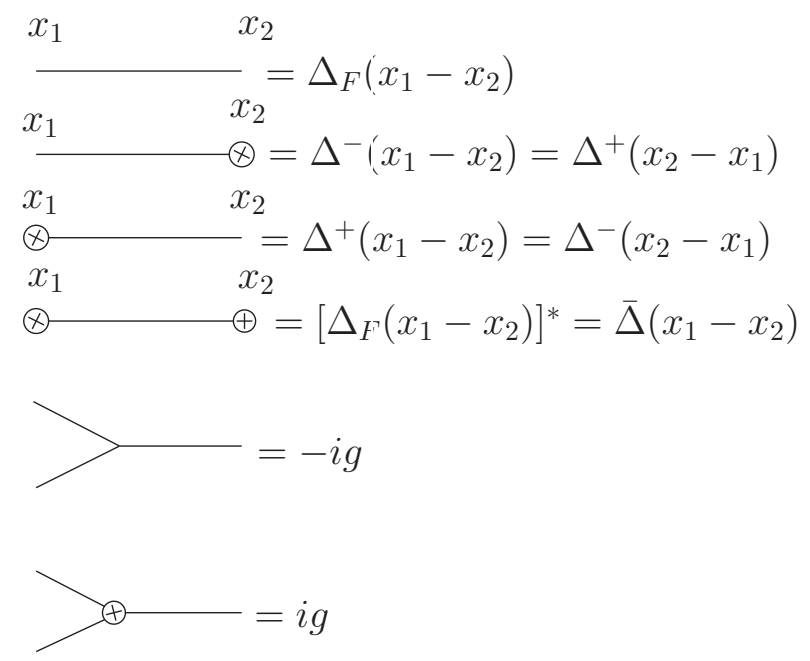

Figura 1.3: Regras de Feynman aumentadas.

$$
\begin{aligned}
& \vec{p} \quad=\frac{i}{p^{2}-m^{2}+i \epsilon} \\
& \vec{p} \quad \oplus=2 \pi \theta\left(-p^{0}\right) \delta\left(p^{2}-m^{2}\right) \\
& \oplus \quad \vec{p} \quad=2 \pi \theta\left(p^{0}\right) \delta\left(p^{2}-m^{2}\right) \\
& \oplus \quad \vec{p} \quad \oplus=\frac{-i}{p^{2}-m^{2}-i \epsilon}
\end{aligned}
$$

Figura 1.4: Regras de Feynman no espaço de momentos. 
Em muitas situações é mais conveniente trabalhar com as regras de Feynman no espaço dos momentos (Figura (1.4)). É importante observar no nosso conjunto de regras, figuras (1.3) e (1.4), que o vértice circulado é justamente o complexo conjugado do vértice original e que a linha com um círculo em cada extremidade é justamente o complexo conjugado do propagador original, ou seja, é o propagador anti-ordenado temporalmente.

Com o conjunto de regras de Feynman aumentadas, podemos desenhar novos tipos de diagramas. Um exemplo é mostrado na figura (1.5). É importante observar que este diagrama é justamente o complexo conjugado daquele mostrado na figura (1.2).

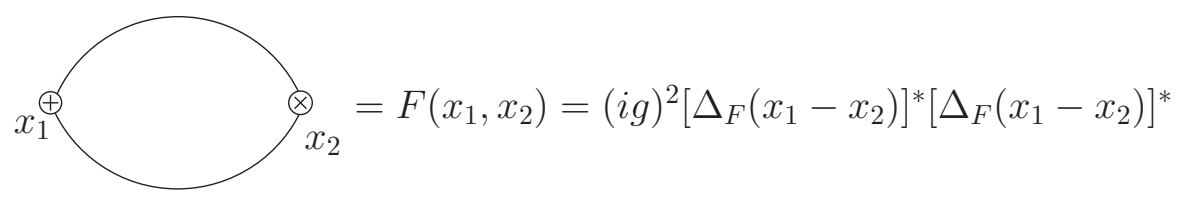

Figura 1.5: Exemplo de amplitude com as regras aumentadas.

De um modo geral, uma amplitude em que todos os vértices estão circulados nada mais é do que o complexo conjugado da amplitude da teoria original (sem vértices circulados), pois os vértices bem como os propagadores são complexos conjugados da teoria original. Para uma dada amplitude, podemos escrever,

$$
F\left(\underline{x_{1}}, \underline{x_{2}}, \cdots, \underline{x_{n}}\right)=\left(F\left(x_{1}, x_{2}, \cdots, x_{n}\right)\right)^{*} .
$$

O traço sob uma coordenada indica que o vértice correspondente é o complexo conjugado, sendo representado como um vértice circulado no diagrama. Então, esse tipo de relação pode fornecer um método diagramático para construir o complexo conjugado, e assim, a parte imaginária de uma amplitude de Feynman da teoria original. Entretanto, esta relação ainda não é uma forma muito simplificada para cálculos.

Vamos explorar as propriedades dos propagadores para encontrar relações mais convenientes. Consideremos as amplitudes mostradas na figura (1.6). Assumindo $x_{1}^{0}>x_{2}^{0} \mathrm{e}$

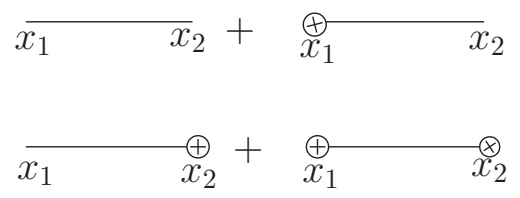

Figura 1.6: Exemplos.

lembrando que cada extremidade das linhas na figura corresponde a um vértice, temos as seguintes expressões,

$$
\Delta_{F}\left(x_{1}-x_{2}\right)+(-) \Delta^{+}\left(x_{1}-x_{2}\right)=\Delta^{+}\left(x_{1}-x_{2}\right)-\Delta^{+}\left(x_{1}-x_{2}\right)=0
$$

e 


$$
(-) \Delta^{-}\left(x_{1}-x_{2}\right)+(-)^{2} \bar{\Delta}\left(x_{1}-x_{2}\right)=-\Delta^{-}\left(x_{1}-x_{2}\right)+\Delta^{-}\left(x_{1}-x_{2}\right)=0 .
$$

Aqui, usamos o fato que um vértice circulado implica num sinal negativo extra e as decomposições (1.6) e (1.10) dos propagadores. Mais um exemplo pode ser visto na figura (1.7). Da mesma forma que o exemplo anterior, considerando $x_{1}^{0}>x_{2}^{0}$, obtemos as
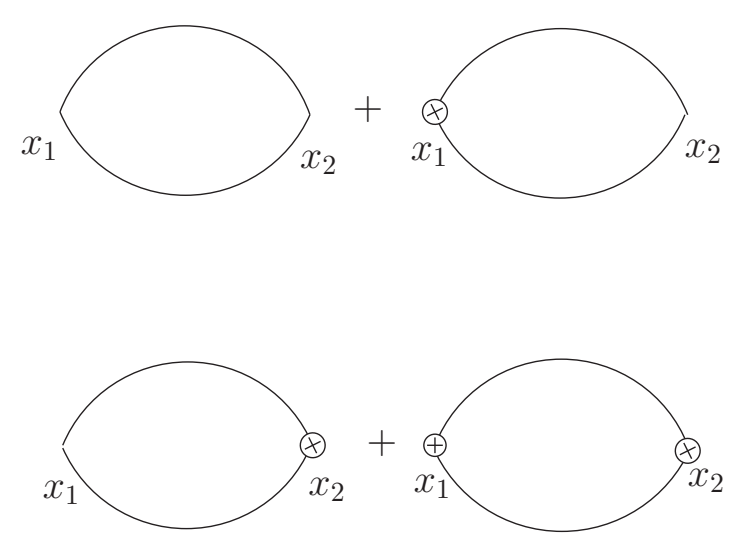

Figura 1.7: Exemplos.

seguintes expressões

$$
\begin{aligned}
& (-i g)^{2}\left(\Delta_{F}\left(x_{1}-x_{2}\right)\right)^{2}+(i g)(-i g)\left(\Delta^{+}\left(x_{1}-x_{2}\right)\right)^{2} \\
= & (-i g)^{2}\left[\left(\Delta^{+}\left(x_{1}-x_{2}\right)\right)^{2}-\left(\Delta^{+}\left(x_{1}-x_{2}\right)\right)^{2}\right]=0
\end{aligned}
$$

e

$$
\begin{aligned}
& (-i g)(i g)\left(\Delta^{-}\left(x_{1}-x_{2}\right)\right)^{2}+(i g)^{2}\left(\bar{\Delta}\left(x_{1}-x_{2}\right)\right)^{2} \\
= & (i g)^{2}\left[-\left(\Delta^{-}\left(x_{1}-x_{2}\right)\right)^{2}+\left(\Delta^{-}\left(x_{1}-x_{2}\right)\right)^{2}\right]=0 .
\end{aligned}
$$

Passemos à uma função de três pontos. Os diagramas relevantes são mostrados na figura (1.8). Tomando $x_{1}^{0}$ como a maior coordenada temporal $\left(x_{1}^{0}>x_{2}^{0}\right.$ e $\left.x_{3}^{0}\right)$, temos as seguintes expressões:

$$
\begin{aligned}
& (-i g)^{3} \Delta_{F}\left(x_{1}-x_{3}\right) \Delta_{F}\left(x_{2}-x_{3}\right) \Delta_{F}\left(x_{1}-x_{2}\right) \\
+ & (i g)(-i g)^{2} \Delta^{+}\left(x_{1}-x_{3}\right) \Delta_{F}\left(x_{2}-x_{3}\right) \Delta^{+}\left(x_{1}-x_{2}\right) \\
= & (-i g)^{3}\left[\Delta^{+}\left(x_{1}-x_{3}\right) \Delta_{F}\left(x_{2}-x_{3}\right) \Delta^{+}\left(x_{1}-x_{2}\right)\right. \\
- & \left.\Delta^{+}\left(x_{1}-x_{3}\right) \Delta_{F}\left(x_{2}-x_{3}\right) \Delta^{+}\left(x_{1}-x_{2}\right)\right]=0
\end{aligned}
$$



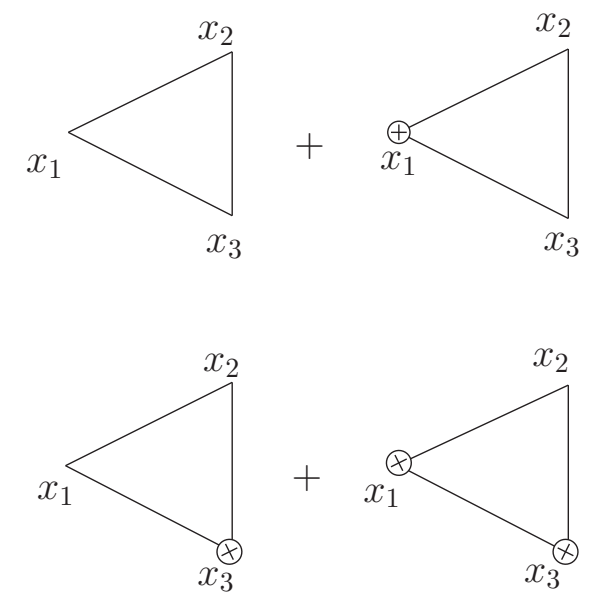

Figura 1.8: Exemplos.

e

$$
\begin{aligned}
& (-i g)^{2}(i g) \Delta^{-}\left(x_{1}-x_{3}\right) \Delta^{-}\left(x_{2}-x_{3}\right) \Delta_{F}\left(x_{1}-x_{2}\right) \\
+ & (i g)^{2}(-i g) \bar{\Delta}\left(x_{1}-x_{3}\right) \Delta^{-}\left(x_{2}-x_{3}\right) \Delta^{+}\left(x_{1}-x_{2}\right) \\
= & (-i g)^{3}\left[-\Delta^{-}\left(x_{1}-x_{3}\right) \Delta^{-}\left(x_{2}-x_{3}\right) \Delta^{+}\left(x_{1}-x_{2}\right)\right. \\
+ & \left.\Delta^{-}\left(x_{1}-x_{3}\right) \Delta^{-}\left(x_{2}-x_{3}\right) \Delta^{+}\left(x_{1}-x_{2}\right)\right]=0 .
\end{aligned}
$$

Dos exemplos mostrados acima podemos tirar uma relação importante. Considerando uma amplitude com o vértice de maior coodenada temporal não circulado e adicionando a ela a correspondente amplitude com o vértice de maior tempo circulado, essa soma é igual a zero. Esta é conhecida como equação do tempo máximo. Uma consequência direta é que para uma amplitude particular, a soma dos gráficos com todas as possíveis maneiras de distribuir os vértices circulados, desde nenhum até todos vértices circulados, é nula. Para entender melhor o que foi dito, consideremos a soma dos diagramas na figura (1.9). A expressão algébrica dessa soma é

$$
\begin{aligned}
& (-i g)^{2}\left(\Delta_{F}\left(x_{1}-x_{2}\right)\right)^{2}+(-i g)(i g)\left(\Delta^{-}\left(x_{1}-x_{2}\right)\right)^{2} \\
+ & (i g)(-i g)\left(\Delta^{+}\left(x_{1}-x_{2}\right)\right)^{2}+(i g)^{2}\left(\bar{\Delta}\left(x_{1}-x_{2}\right)\right)^{2} \\
= & (-i g)^{2}\left[\theta\left(x_{1}^{0}-x_{2}^{0}\right)\left(\Delta^{+}\left(x_{1}-x_{2}\right)\right)^{2}+\theta\left(x_{2}^{0}-x_{1}^{0}\right)\left(\Delta^{-}\left(x_{1}-x_{2}\right)\right)^{2}\right. \\
- & \left(\Delta^{-}\left(x_{1}-x_{2}\right)\right)^{2}-\left(\Delta^{+}\left(x_{1}-x_{2}\right)\right)^{2}+\theta\left(x_{1}^{0}-x_{2}^{0}\right)\left(\Delta^{-}\left(x_{1}-x_{2}\right)\right)^{2} \\
+ & \left.\theta\left(x_{2}^{0}-x_{1}^{0}\right)\left(\Delta^{+}\left(x_{1}-x_{2}\right)\right)^{2}\right) \\
= & 0 .
\end{aligned}
$$

Então, dada uma soma com todas as possibilidades para os vértices circulados, diagramas com o vértice de maior tempo circulado juntamente com os diagramas correspondentes 

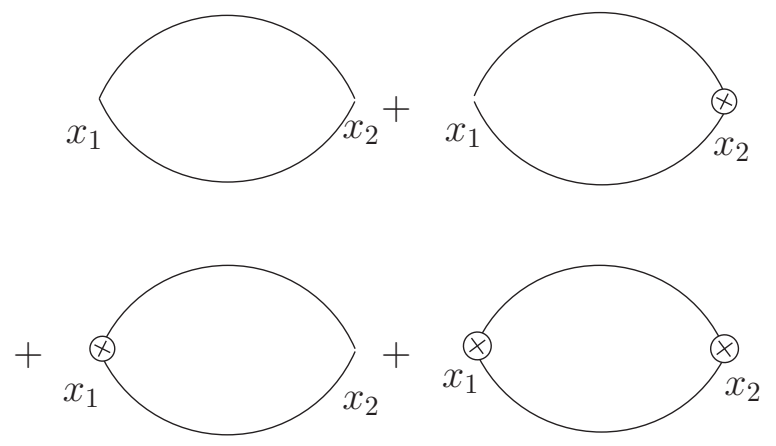

Figura 1.9: Exemplo.

sem esse vértice circulado se anulam devido a equação do tempo máximo. Os diagramas se cancelam aos pares. Notemos que essa soma sempre tem um número par de termos. Vale dizer que todas essas propriedades permanecem verdadeiras no espaço dos momentos.

Para uma certa amplitude $F$, com $n$ vértices, podemos escrever

$$
\sum_{\text {Todas possibilidades }} F\left(x_{1}, x_{2}, \cdots, \underline{x_{i}}, \cdots, x_{n}\right)=0 .
$$

Separando os termos com nenhum vértice circulado e com todos os vértices circulados, (1.22) torna-se,

$$
F\left(x_{1}, x_{2}, \cdots, x_{n}\right)+F\left(\underline{x_{1}}, \underline{x_{2}}, \cdots, \underline{x_{n}}\right)=-\sum^{\prime} F\left(x_{1}, x_{2}, \cdots, \underline{x_{i}}, \cdots, x_{n}\right),
$$

com a linha de $\sum^{\prime}$ indicando a ausência dos termos que foram separados. De acordo com a nossa definição da matriz $T, S=1+i T$, com um fator de $i$ extra, tal que

$$
F \sim i T
$$

vemos que a parte real de um diagrama contribui para a parte imaginária da matriz $T$. Dessa forma, podemos relacionar o lado esquerdo de (1.23) com a parte imaginária da matriz $T$, que por sua vez pode ser expressa diagramaticamente em termos dos gráficos com todas as possibilidades de circular os vértices. O lado direito de (1.23) ainda envolve um grande número de diagramas, não sendo muito simples para fins de cálculos. Além disso, ele não está numa forma em que a relação de unitariedade (1.4) é manifesta.

Agora, vamos investigar algumas simplificações que podem ocorrer no lado direito de (1.23). Para isto, consideremos a figura (1.10). De acordo com as regras de Feynman dadas no espaço dos momentos, a expressão associada a esse diagrama contém os seguintes propagadores:

$$
\sim \Delta^{-}\left(k_{1}\right) \Delta^{-}\left(k_{2}\right) \Delta^{-}\left(k_{3}\right) \sim \theta\left(-k_{1}^{0}\right) \theta\left(-k_{2}^{0}\right) \theta\left(-k_{3}^{0}\right)=0
$$




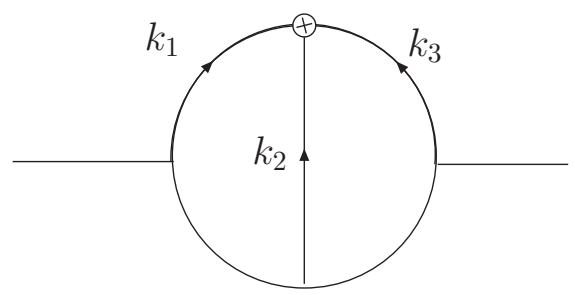

Figura 1.10: Exemplo.

$\mathrm{Na}$ última igualdade usamos a conservação de energia-momento $k_{1}+k_{2}+k_{3}=0$, que gera uma contradição entre as funções $\theta$. Esta análise mostra que qualquer diagrama de Feynman com um vértice circulado isolado é nulo. Da mesma maneira, qualquer diagrama com um vértice não circulado isolado também é nulo, pois esse é justamente o complexo conjugado do primeiro, que já era nulo. Esse fato elimina alguns diagramas na soma em (1.23).

Prosseguindo nossa análise, vamos convencionar que em um diagrama, partículas incidem a partir da esquerda e emergem pela direita, quer dizer, o fluxo de energia-momento é para direita. Sob essa condição, sempre que um vértice não circulado está ligado às linhas externas à esquerda e rodeado por vértices circulados, esse diagrama é nulo. Para ilustrar esse fato, examinemos a figura (1.11). A parte referente as funções $\theta$ associadas

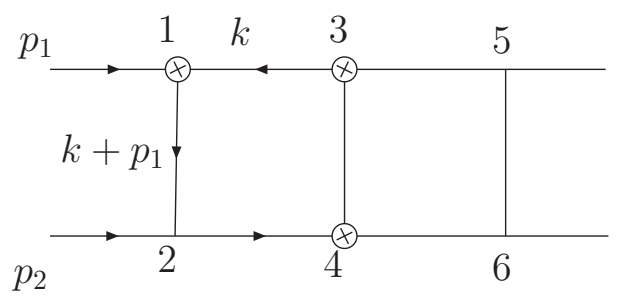

Figura 1.11: Exemplo.

a esse gráfico é

$$
\sim \theta\left(k^{0}+p_{1}^{0}\right) \theta\left(-k^{0}-p_{1}^{0}-p_{2}^{0}\right)=0,
$$

que é nulo pois $p_{1}^{0}$ e $p_{2}^{0}$ são positivos, gerando um conflito entre os argumentos das funções $\theta$.

Com o que foi discutido acima, vemos que os diagramas que contribuem para o lado direito de (1.23) são aqueles em que os vértices circulados, assim como os não circulados, formam regiões conexas, ligadas as linhas externas e sem vértices isolados. Com a nossa convenção, os vértices circulados estarão ligados as linhas externas pela esquerda e os não circulados pela direita.

Devido ao tipo dos gráficos que contribuem em (1.23), ou seja, aqueles em que os vértices circulados e não circulados formam regiões conexas, podemos introduzir a des- 
crição de corte dos diagramas. Essa descrição é exemplificada nos diagramas da figura (1.12). No diagrama cortado, a região à esquerda da linha de corte (tracejada) é entendida como a região em que todos os vérices estão circulados. Visto que todos os vértices
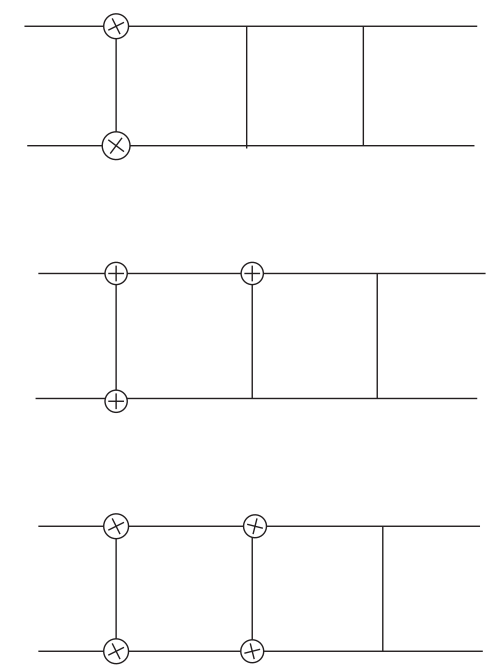
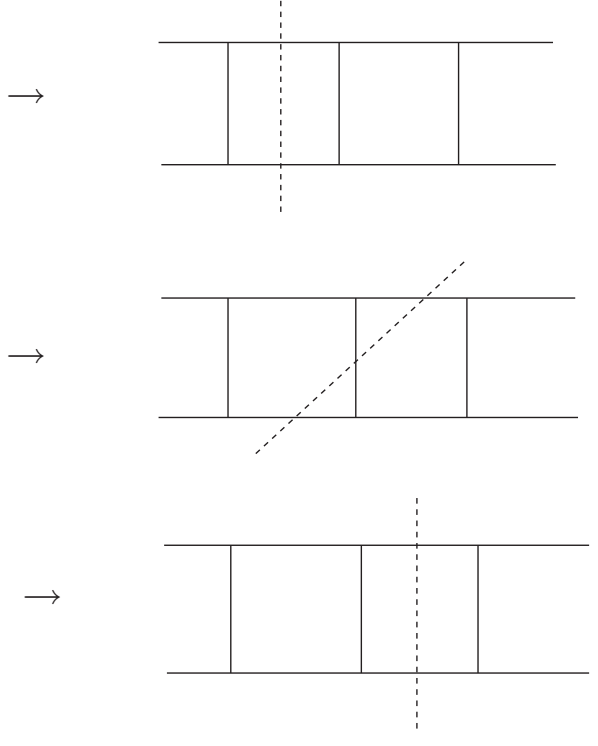

Figura 1.12: Descrição de corte.

à esquerda dessa linha correspondem ao complexo conjugado dos vértices e propagadores da teoria original, essa parte do diagrama pode ser pensada como o complexo conjugado de um elemento de matriz da teoria original. Então, podemos escrever a parte imaginária de uma amplitude de Feynman em termos dos diagramas cortados, os quais também conduzem a uma interpretação natural em termos da unitariedade. Exemplos são mostrados nas figuras (1.13) e (1.14).

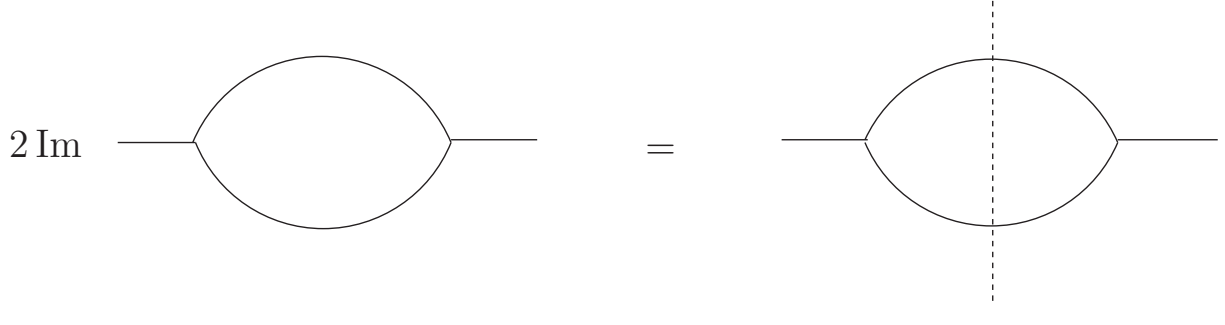

Figura 1.13: Regra de corte

Essas são as regras de corte. Elas dizem que a parte imaginária de uma amplitude de Feynman é dada pela soma de todos os possíveis cortes do correspondente diagrama, os quais separam os vértices circulados dos não circulados tal que não existam regiões isoladas de vértices circulados ou não. 


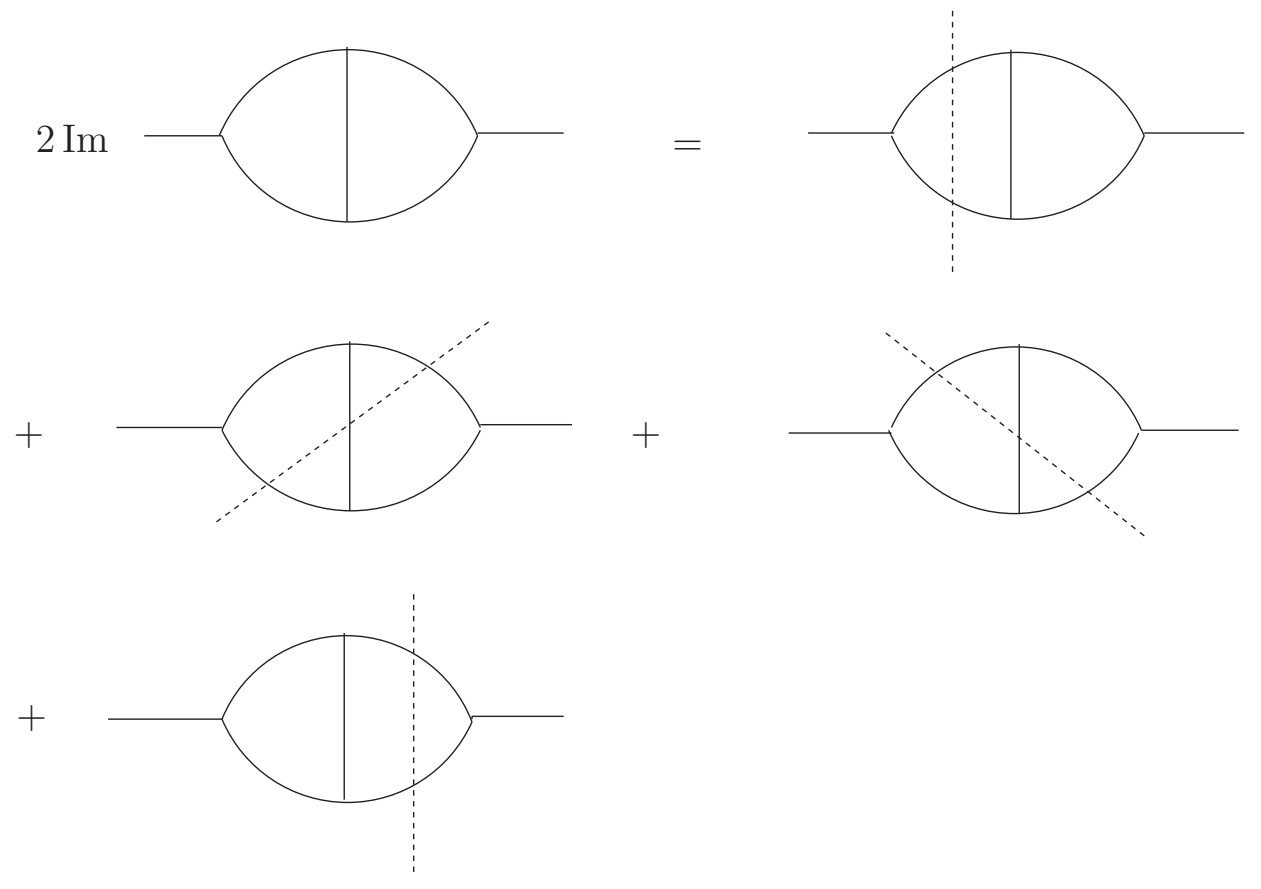

Figura 1.14: Regra de corte.

Antes de partir para o estudo da unitariedade em alguns modelos da teoria de campos não comutativa, apresentaremos uma breve introdução sobre o assunto, que é o tema abordado na seção seguinte.

\subsection{Não Comutatividade}

O princípio básico para introduzir um espaço não comutativo é estabelecer uma regra de comutação entre operadores de posição, ou seja,

$$
\left[q^{\mu}, q^{\nu}\right]=i \Theta^{\mu \nu}
$$

sendo $\Theta^{\mu \nu}$ uma matriz anti-simétrica e, por simplicidade, assumiremos que comuta com operadores de posição. Poderíamos conduzir nossos cálculos sempre levando em conta essa regra de comutação. Entretanto, essa maneira não é adequada. Um modo conveniente para tratar esse problema é o procedimento de Weyl-Moyal. Nele, de forma equivalente, usamos operadores de posição que comutam entre si, porém, o produto ordinário é substituído pelo produto Moyal. Esse procedimento pode ser interpretado como uma deformação do espaço.

No contexto de Weyl-Moyal, iniciamos nosso estudo introduzindo o operador 


$$
T(k)=e^{i k_{\mu} q^{\mu}}
$$

Notemos que ele satisfaz as seguintes propriedades:

$$
T^{\dagger}(k)=e^{-i k_{\mu}\left(q^{\mu}\right)^{\dagger}}=T(-k) \text { se } q^{\mu \dagger}=q^{\mu}
$$

e

$$
T(k) T\left(k^{\prime}\right)=T\left(k+k^{\prime}\right) e^{-\frac{i}{2} k_{\mu} k_{\nu}^{\prime} \Theta^{\mu \nu}} .
$$

Esta última propriedade pode ser obtida empregando o teorema BCH (Baker-CampbellHausdorff), isto é,

$$
e^{A} e^{B}=\exp \left[A+B+\frac{1}{2}[A, B]+\frac{1}{12}([A,[A, B]]-[B,[A, B]])+\cdots\right] .
$$

Identificando $A=i k_{\mu} q^{\mu}$ e $B=i k_{\nu}^{\prime} q^{\nu}$, obtemos,

$$
\begin{aligned}
T(k) T\left(k^{\prime}\right) & =e^{i k_{\mu} q^{\mu}+i k_{\nu}^{\prime} q^{\nu}} e^{-\frac{i}{2} k_{\mu} k_{\nu}^{\prime} \Theta^{\mu \nu}} \\
& =T\left(k+k^{\prime}\right) e^{-\frac{i}{2} k_{\mu} k_{\nu}^{\prime} \Theta^{\mu \nu}}
\end{aligned}
$$

lembrando que estamos considerando que $\Theta^{\mu \nu}$ comuta com operadores de posição. Consideremos também o traço de $T(k)$, que é definido por meio de

$$
\operatorname{Tr} T(k) \equiv(2 \pi)^{D} \prod_{\mu} \delta^{D}\left(k_{\mu}\right)
$$

É possível associar a uma dada função clássica, $\phi(x)$, um operador, $\Phi$. Assim, inspirado na integral de Fourier, podemos convenientemente estabelecer essa conexão via

$$
\begin{aligned}
\Phi & =\frac{1}{(2 \pi)^{D}} \int d^{D} x d^{D} k T(k) e^{i k_{\mu} x^{\mu}} \phi(x) \\
& =\int \frac{d^{D} k}{(2 \pi)^{D}} T(k) \widetilde{\phi}(k),
\end{aligned}
$$

em que

$$
\widetilde{\phi}(k)=\int d^{D} x e^{i k x} \phi(x) .
$$

A relação inversa de (1.34) é obtida multiplicando-a por $T^{\dagger}\left(k^{\prime}\right)$ e tomando o traço, isto é, 


$$
\begin{aligned}
\operatorname{Tr}\left[\Phi T^{\dagger}\left(k^{\prime}\right)\right] & =\frac{1}{(2 \pi)^{D}} \int d^{D} x d^{D} k \operatorname{Tr}\left[T(k) T^{\dagger}\left(k^{\prime}\right)\right] e^{i k x} \phi(x) \\
& =\frac{1}{(2 \pi)^{D}} \int d^{D} x d^{D} k \operatorname{Tr}\left[T\left(k-k^{\prime}\right)\right] e^{\frac{i}{2} k_{\mu} k_{\nu}^{\prime} \Theta^{\mu \nu}} e^{i k x} \phi(x) \\
& =\int d^{D} x d^{D} k \delta\left(k-k^{\prime}\right) e^{\frac{i}{2} k_{\mu} k_{\nu}^{\prime} \Theta^{\mu \nu}} e^{i k x} \phi(x) \\
& =\int d^{D} x e^{i k^{\prime} x} \phi(x),
\end{aligned}
$$

sendo que usamos $(1.29),(1.30),(1.33)$ e o fato que $k_{\mu} k_{\nu} \Theta^{\mu \nu}=0$, ou seja, o produto de um tensor simétrico por um anti-simétrico é nulo. Em seguida, basta tomar a transformada inversa de (1.36), resultando em

$$
\phi(x)=\int \frac{d^{D} k}{(2 \pi)^{D}} e^{-i k x} \operatorname{Tr}\left[\Phi T^{\dagger}(k)\right] .
$$

A partir dessa expressão, podemos construir o produto Moyal de funções clássicas, $\phi_{1}$ e $\phi_{2}$, correspondendo ao produto dos operadores $\Phi_{1}$ e $\Phi_{2}$. Desse modo, definimos o produto Moyal como segue:

$$
\phi_{1}(x) * \phi_{2}(x)=\int \frac{d^{D} k}{(2 \pi)^{D}} e^{-i k x} \operatorname{Tr}\left[\Phi_{1} \Phi_{2} T^{\dagger}(k)\right] .
$$

Usando (1.34), podemos escrever a equação acima de outra forma conveniente. Então,

$$
\begin{aligned}
\phi_{1}(x) * \phi_{2}(x) & =\int \frac{d^{D} k}{(2 \pi)^{D}} \frac{d^{D} k_{1}}{(2 \pi)^{D}} \frac{d^{D} k_{2}}{(2 \pi)^{D}} e^{-i k x} \widetilde{\phi}_{1}\left(k_{1}\right) \widetilde{\phi}_{2}\left(k_{2}\right) \operatorname{Tr}\left[T\left(k_{1}\right) T\left(k_{2}\right) T^{\dagger}(k)\right] \\
& =\int \frac{d^{D} k}{(2 \pi)^{D}} \frac{d^{D} k_{1}}{(2 \pi)^{D}} \frac{d^{D} k_{2}}{(2 \pi)^{D}} e^{-i k x} \widetilde{\phi}_{1}\left(k_{1}\right) \widetilde{\phi}_{2}\left(k_{2}\right) e^{-\frac{i}{2} k_{1}^{\mu} k_{2}^{\nu} \Theta_{\mu \nu}} \operatorname{Tr}\left[T\left(k_{1}+k_{2}\right) T(-k)\right] \\
& =\int \frac{d^{D} k}{(2 \pi)^{D}} \frac{d^{D} k_{1}}{(2 \pi)^{D}} \frac{d^{D} k_{2}}{(2 \pi)^{D}} e^{-i k x} \widetilde{\phi}_{1}\left(k_{1}\right) \widetilde{\phi}_{2}\left(k_{2}\right) e^{-\frac{i}{2} k_{1}^{\mu} k_{2}^{\nu} \Theta_{\mu \nu}} e^{\frac{i}{2}\left(k_{1}+k_{2}\right)^{\mu} k^{\nu} \Theta_{\mu \nu}} \\
& \times(2 \pi)^{D} \delta^{D}\left(k_{1}+k_{2}-k\right) \\
& =\int \frac{d^{D} k_{1}}{(2 \pi)^{D}} \frac{d^{D} k_{2}}{(2 \pi)^{D}} e^{-i\left(k_{1}+k_{2}\right) x} \widetilde{\phi}_{1}\left(k_{1}\right) \widetilde{\phi}_{2}\left(k_{2}\right) e^{-\frac{i}{2} k_{1}^{\mu} k_{2}^{\nu} \Theta_{\mu \nu}} \\
& =\lim _{y \rightarrow x} e^{\frac{i}{2} \Theta^{\mu \nu} \frac{\partial}{\partial y^{\mu}} \frac{\partial}{\partial x^{\mu}}} \phi_{1}(y) \phi_{2}(x) .
\end{aligned}
$$

Esse resultado mostra que o produto Moyal é altamente não local, dependendo de um número arbitrário de derivadas. É oportuno nesse ponto a seguinte observação: justamente a não localidade da coordenada temporal é que traz problemas referentes à unitariedade. Veremos esse fato explicitamente no estudo de alguns modelos nos próximos capítulos. 
Uma observação importante é que o comutador entre as coordenadas (ordinárias) $x^{\mu}$ e $x^{\nu}$, com produto Moyal no lugar do produto ordinário, reproduz a equação (1.27), ou seja, $\left[x^{\mu}, x^{\nu}\right]_{*}=i \Theta^{\mu \nu}$. Isso mostra que o produto Moyal entre variáveis que comutam é equivalente ao produto ordinário entre operadores que não comutam tal como definido em (1.27).

Consideremos agora, a integral do produto Moyal de dois campos,

$$
\begin{aligned}
\int d^{D} x \phi_{1}(x) * \phi_{2}(x) & =\int \frac{d^{D} k}{(2 \pi)^{D}} \int d x e^{-i k x} \operatorname{Tr}\left[\Phi_{1} \Phi_{2} T^{\dagger}(k)\right] \\
& =\int d^{D} k \delta^{D}(k) \operatorname{Tr}\left[\Phi_{1} \Phi_{2} T^{\dagger}(k)\right] \\
& =\operatorname{Tr}\left[\Phi_{1} \Phi_{2}\right] .
\end{aligned}
$$

A generalização para o produto de $n$ campos é imediata:

$$
\int d^{D} x \phi_{1}(x) * \phi_{2}(x) * \cdots * \phi_{n}(x)=\operatorname{Tr}\left[\Phi_{1} \Phi_{2} \cdots \Phi_{n}\right] .
$$

Portanto, o produto Moyal não é alterado por permutações cíclicas dos campos. Retornando à equação (1.40), podemos escrevê-la, com a ajuda de (1.34) e (1.35), da seguinte maneira:

$$
\begin{aligned}
\int d^{D} x \phi_{1}(x) * \phi_{2}(x) & =\operatorname{Tr}\left[\Phi_{1} \Phi_{2}\right] \\
& =\int \frac{d^{D} k_{1}}{(2 \pi)^{D}} \frac{d^{D} k_{2}}{(2 \pi)^{D}} \operatorname{Tr}\left[T\left(k_{1}\right) T\left(k_{2}\right)\right] \widetilde{\phi}_{1}\left(k_{1}\right) \widetilde{\phi}_{2}\left(k_{2}\right) \\
& =\int \frac{d^{D} k_{1}}{(2 \pi)^{D}} \frac{d^{D} k_{2}}{(2 \pi)^{D}}(2 \pi)^{D} \delta^{D}\left(k_{1}+k_{2}\right) e^{-\frac{i}{2} k_{1}^{\mu} k_{2}^{\nu} \Theta_{\mu \nu}} \widetilde{\phi}_{1}\left(k_{1}\right) \widetilde{\phi}_{2}\left(k_{2}\right) \\
& =\int \frac{d^{D} k_{1}}{(2 \pi)^{D}} \widetilde{\phi}_{1}\left(k_{1}\right) \widetilde{\phi}_{2}\left(-k_{1}\right) \\
& =\int d^{D} x \phi_{1}(x) \phi_{2}(x),
\end{aligned}
$$

mostrando que a parte quadrática da ação de uma teoria de campos não é alterada quando passamos de um produto ordinário a um produto Moyal. Na sequência, analisemos o caso de três campos, que é obtido por um procedimento semelhante ao anterior, 


$$
\begin{aligned}
& \int d^{D} x \phi_{1}(x) * \phi_{2}(x) * \phi_{3}(x)=\operatorname{Tr}\left[\Phi_{1} \Phi_{2} \Phi_{3}\right] \\
= & \int \prod_{i=1}^{3} \frac{d^{D} k_{i}}{(2 \pi)^{D}} \operatorname{Tr}\left[T\left(k_{1}\right) T\left(k_{2}\right) T\left(k_{3}\right)\right] \widetilde{\phi}_{1}\left(k_{1}\right) \widetilde{\phi}_{2}\left(k_{2}\right) \widetilde{\phi}_{3}\left(k_{3}\right) \\
= & \int \prod_{i=1}^{3} \frac{d^{D} k_{i}}{(2 \pi)^{D}} \operatorname{Tr}\left[T\left(k_{1}+k_{2}+k_{3}\right)\right] e^{-\frac{i}{2}\left(k_{1}+k_{2}\right)^{\mu} k_{3}^{\nu} \Theta_{\mu \nu}} e^{-\frac{i}{2} k_{1}^{\mu} k_{2}^{\nu} \Theta_{\mu \nu}} \widetilde{\phi}_{1}\left(k_{1}\right) \widetilde{\phi}_{2}\left(k_{2}\right) \widetilde{\phi}_{3}\left(k_{3}\right) \\
= & \int \prod_{i=1}^{3} \frac{d^{D} k_{i}}{(2 \pi)^{D}}(2 \pi)^{D} \delta^{D}\left(k_{1}+k_{2}+k_{3}\right) e^{-\frac{i}{2}\left(k_{1}+k_{2}\right)^{\mu} k_{3}^{\nu} \Theta_{\mu \nu}} e^{-\frac{i}{2} k_{1}^{\mu} k_{2}^{\nu} \Theta_{\mu \nu}} \widetilde{\phi}_{1}\left(k_{1}\right) \widetilde{\phi}_{2}\left(k_{2}\right) \widetilde{\phi}_{3}\left(k_{3}\right) \\
= & \int \prod_{i=1}^{3} \frac{d^{D} k_{i}}{(2 \pi)^{D}}(2 \pi)^{D} \delta^{D}\left(k_{1}+k_{2}+k_{3}\right) e^{-\frac{i}{2} k_{1}^{\mu} k_{2}^{\nu} \Theta_{\mu \nu}} \widetilde{\phi}_{1}\left(k_{1}\right) \widetilde{\phi}_{2}\left(k_{2}\right) \widetilde{\phi}_{3}\left(k_{3}\right) .
\end{aligned}
$$

Esse resultado conduz a generalização para $m$ campos,

$$
\begin{aligned}
& \int d^{D} x \phi_{1}(x) * \phi_{2}(x) * \cdots * \phi_{m}(x) \\
= & \int \prod_{i=1}^{m} \frac{d^{D} k_{i}}{(2 \pi)^{D}}(2 \pi)^{D} \delta^{D}\left(k_{1}+k_{2}+\cdots+k_{m}\right) \widetilde{\phi}_{1}\left(k_{1}\right) \widetilde{\phi}_{2}\left(k_{2}\right) \cdots \widetilde{\phi}_{m}\left(k_{m}\right) \\
\times & \exp \left(-\frac{i}{2} \sum_{i<j} k_{i} \wedge k_{j}\right)
\end{aligned}
$$

em que empregamos a notação compacta $k_{1} \wedge k_{2} \equiv k_{1}^{\mu} k_{2}^{\nu} \Theta_{\mu \nu}$.

Quando alguns dos campos em (1.44) são iguais, certas simplificações ocorrem. Veremos a seguir alguns exemplos específicos. No primeiro deles, vamos considerar a equação (1.43), porém, com dois dos campos iguais, isto é,

$$
\begin{aligned}
& \int d^{D} x \phi_{1}(x) * \phi_{1}(x) * \phi_{2}(x) \\
= & \int \frac{d^{D} k_{1}}{(2 \pi)^{D}} \frac{d^{D} k_{2}}{(2 \pi)^{D}} \frac{d^{D} k_{3}}{(2 \pi)^{D}}(2 \pi)^{D} \delta^{D}\left(k_{1}+k_{2}+k_{3}\right) e^{-\frac{i}{2} k_{1} \wedge k_{2}} \widetilde{\phi}_{1}\left(k_{1}\right) \widetilde{\phi}_{1}\left(k_{2}\right) \widetilde{\phi}_{2}\left(k_{3}\right) \\
= & \int \frac{d^{D} k_{1}}{(2 \pi)^{D}} \frac{d^{D} k_{2}}{(2 \pi)^{D}} \frac{d^{D} k_{3}}{(2 \pi)^{D}}(2 \pi)^{D} \delta^{D}\left(k_{1}+k_{2}+k_{3}\right) \frac{e^{-\frac{i}{2} k_{1} \wedge k_{2}}+e^{-\frac{i}{2} k_{2} \wedge k_{1}}}{2} \widetilde{\phi}_{1}\left(k_{1}\right) \widetilde{\phi}_{1}\left(k_{2}\right) \widetilde{\phi}_{2}\left(k_{3}\right) \\
= & \int \frac{d^{D} k_{1}}{(2 \pi)^{D}} \frac{d^{D} k_{2}}{(2 \pi)^{D}} \frac{d^{D} k_{3}}{(2 \pi)^{D}}(2 \pi)^{D} \delta^{D}\left(k_{1}+k_{2}+k_{3}\right) \cos \left(\frac{k_{1} \wedge k_{2}}{2}\right) \widetilde{\phi}_{1}\left(k_{1}\right) \widetilde{\phi}_{1}\left(k_{2}\right) \widetilde{\phi}_{2}\left(k_{3}\right),
\end{aligned}
$$


observando que $k_{1} \wedge k_{2}=-k_{2} \wedge k_{1}$. Nos demais exemplos apresentaremos apenas os resultados, sendo que os detalhes dos cálculos são deixados para o apêndice B.

O exemplo seguinte é o caso de quatro campos sendo que dois a dois são iguais,

$$
\begin{aligned}
& \int d^{D} x \phi_{1}(x) * \phi_{1}(x) * \phi_{2}(x) * \phi_{2}(x) \\
= & \int \prod_{i=1}^{4} \frac{d^{D} k_{i}}{(2 \pi)^{D}}(2 \pi)^{D} \delta^{D}\left(k_{1}+k_{2}+k_{3}+k_{4}\right) \\
\times & \cos \left(\frac{k_{1} \wedge k_{2}}{2}\right) \cos \left(\frac{k_{3} \wedge k_{4}}{2}\right) \widetilde{\phi}_{1}\left(k_{1}\right) \widetilde{\phi}_{1}\left(k_{2}\right) \widetilde{\phi}_{2}\left(k_{3}\right) \widetilde{\phi}_{2}\left(k_{4}\right) .
\end{aligned}
$$

No caso semelhante ao anterior, porém com os campos iguais alternados, temos

$$
\begin{aligned}
& \int d^{D} x \phi_{1}(x) * \phi_{2}(x) * \phi_{1}(x) * \phi_{2}(x) \\
= & \int \prod_{i=1}^{4} \frac{d^{D} k_{i}}{(2 \pi)^{D}}(2 \pi)^{D} \delta^{D}\left(k_{1}+k_{2}+k_{3}+k_{4}\right) \\
\times & \cos \left(\frac{k_{1} \wedge k_{2}+k_{3} \wedge k_{4}}{2}\right) \widetilde{\phi}_{1}\left(k_{1}\right) \widetilde{\phi}_{2}\left(k_{2}\right) \widetilde{\phi}_{1}\left(k_{3}\right) \widetilde{\phi}_{2}\left(k_{4}\right) .
\end{aligned}
$$

Vale ressaltar que os casos anteriores referem-se a campos bosônicos. No caso de campos fermiônicos, teremos que levar em conta o caráter anti-comutativo dos mesmos. Ainda que estamos no contexto clássico (antes da quantização), o reflexo desse fato sobre os cálculos é que devemos tratar os campos fermiônicos como variáveis de Grassmann, isto é, variáveis que anti-comutam entre si.

Dessa forma, considerando em (1.46) campos fermiônicos no lugar de campos bosônicos, obtemos o seguinte resultado,

$$
\begin{aligned}
& \int d^{D} x \bar{\psi}_{1}(x) * \bar{\psi}_{1}(x) * \psi_{2}(x) * \psi_{2}(x) \\
= & -\int \prod_{i=1}^{4} \frac{d^{D} k_{i}}{(2 \pi)^{D}}(2 \pi)^{D} \delta^{D}\left(k_{1}+k_{2}+k_{3}+k_{4}\right) \\
\times & \sin \left(\frac{k_{1} \wedge k_{2}}{2}\right) \sin \left(\frac{k_{3} \wedge k_{4}}{2}\right) \widetilde{\bar{\psi}}_{1}\left(k_{1}\right) \widetilde{\bar{\psi}}_{1}\left(k_{2}\right) \widetilde{\psi}_{2}\left(k_{3}\right) \widetilde{\psi}_{2}\left(k_{4}\right) .
\end{aligned}
$$


Por fim, vamos considerar campos fermiônicos em (1.47),

$$
\begin{aligned}
& \int d^{D} x \bar{\psi}_{1}(x) * \psi_{2}(x) * \bar{\psi}_{1}(x) * \psi_{2}(x) \\
= & -i \int \prod_{i=1}^{4} \frac{d^{D} k_{i}}{(2 \pi)^{D}}(2 \pi)^{D} \delta^{D}\left(k_{1}+k_{2}+k_{3}+k_{4}\right) \\
\times & \sin \left(\frac{k_{1} \wedge k_{2}+k_{3} \wedge k_{4}}{2}\right) \widetilde{\bar{\psi}}_{1}\left(k_{1}\right) \widetilde{\psi}_{2}\left(k_{2}\right) \widetilde{\bar{\psi}}_{1}\left(k_{3}\right) \widetilde{\psi}_{2}\left(k_{4}\right) .
\end{aligned}
$$

Os detalhes desses cálculos envolvendo campos fermiônicos também são mostrados no apêndice B.

Após as discussões feitas ao longo desse capítulo, estamos em condições de partir para o estudo de modelos específicos de teorias não comutativas no que se refere à unitariedade. Isso será feito a partir do próximo capítulo. 


\section{Capítulo 2}

\section{Unitariedade no modelo $\phi^{3}$ não comutativo}

Neste capítulo, vamos empregar as regras de corte para estudar o problema da unitariedade no modelo $\phi^{3}$ não comutativo. Esse estudo será dividido essencialmente em dois casos distintos: não comutatividade envolvendo apenas o espaço e a não comutatividade envolvendo o espaço e o tempo. Veremos que esses dois casos conduzem a resultados muito diferentes.

\section{$2.1 \quad$ Modelo $\phi^{3}$ Não Comutativo}

O modelo que vamos estudar é descrito pela langrangiana

$$
\mathcal{L}=\frac{1}{2} \partial_{\mu} \phi \partial^{\mu} \phi-\frac{m^{2}}{2} \phi^{2}-\frac{\lambda}{3 !} \phi * \phi * \phi .
$$

O primeiro passo é determinar as regras de Feynman para esse modelo pois, dado um diagrama, podemos imediatamente escrever a expressão algébrica associada a ele. O fator de vértice é obtido a partir da contribuição perturbativa de ordem mais baixa não trivial para a função de três pontos, ou seja, devemos calcular

$$
V_{3} \equiv\left\langle 0\left|T \phi\left(x_{1}\right) \phi\left(x_{2}\right) \phi\left(x_{3}\right)\left(\frac{-i \lambda}{3 !} \int d^{D} x \phi(x) * \phi(x) * \phi(x)\right)\right| 0\right\rangle .
$$

A idéia é escrever o produto Moyal dos três campos acima em termos do produto ordinário desses campos, pois assim podemos usar o teorema de Wick para calcular o valor esperado no vácuo. Para isto, usamos a equação (1.45) seguida de (1.35). Obtemos assim, 


$$
\begin{aligned}
V_{3} & =\frac{-i \lambda}{3 !} \int \frac{d^{D} k_{1}}{(2 \pi)^{D}} \frac{d^{D} k_{2}}{(2 \pi)^{D}} \frac{d^{D} k_{3}}{(2 \pi)^{D}}(2 \pi)^{D} \delta^{D}\left(k_{1}+k_{2}+k_{3}\right) \cos \left(\frac{k_{1} \wedge k_{2}}{2}\right) \\
& \times \int d^{D} y_{1} d^{D} y_{2} d^{D} y_{3} e^{i k_{1} y_{1}} e^{i k_{2} y_{2}} e^{i k_{3} y_{3}}\left\langle 0\left|T\left[\phi\left(x_{1}\right) \phi\left(x_{2}\right) \phi\left(x_{3}\right) \phi\left(y_{1}\right) \phi\left(y_{2}\right) \phi\left(y_{3}\right)\right]\right| 0\right\rangle \\
& =-i \lambda \int \frac{d^{D} k_{1}}{(2 \pi)^{D}} \frac{d^{D} k_{2}}{(2 \pi)^{D}} \frac{d^{D} k_{3}}{(2 \pi)^{D}}(2 \pi)^{D} \delta^{D}\left(k_{1}+k_{2}+k_{3}\right) \cos \left(\frac{k_{1} \wedge k_{2}}{2}\right) \\
& \times \int d^{D} y_{1} d^{D} y_{2} d^{D} y_{3} e^{i k_{1} y_{1}} e^{i k_{2} y_{2}} e^{i k_{3} y_{3}} \Delta_{F}\left(x_{1}-y_{1}\right) \Delta_{F}\left(x_{2}-y_{2}\right) \Delta_{F}\left(x_{3}-y_{3}\right) .
\end{aligned}
$$

Agora, vamos escrever essa expressão no espaço de momentos. Isso é feito tomando a transformada de Fourier e também escrevendo os propagadores em termos de suas transformadas:

$$
\begin{aligned}
\widetilde{V}_{3} & =-i \lambda \int d^{D} x_{1} d^{D} x_{2} d^{D} x_{3} e^{i p_{1} x_{1}} e^{i p_{2} x_{2}} e^{i p_{3} x_{3}} \int \frac{d^{D} k_{1}}{(2 \pi)^{D}} \frac{d^{D} k_{2}}{(2 \pi)^{D}} \frac{d^{D} k_{3}}{(2 \pi)^{D}}(2 \pi)^{D} \delta^{D}\left(k_{1}+k_{2}+k_{3}\right) \\
& \times \cos \left(\frac{k_{1} \wedge k_{2}}{2}\right) \int d^{D} y_{1} d^{D} y_{2} d^{D} y_{3} e^{i k_{1} y_{1}} e^{i k_{2} y_{2}} e^{i k_{3} y_{3}} \int \frac{d^{D} q_{1}}{(2 \pi)^{D}} e^{-i q_{1}\left(x_{1}-y_{1}\right)} \widetilde{\Delta}_{F}\left(q_{1}\right) \\
& \times \int \frac{d^{D} q_{2}}{(2 \pi)^{D}} e^{-i q_{2}\left(x_{2}-y_{2}\right)} \widetilde{\Delta}_{F}\left(q_{2}\right) \int \frac{d^{D} q_{3}}{(2 \pi)^{D}} e^{-i q_{3}\left(x_{3}-y_{3}\right)} \widetilde{\Delta}_{F}\left(q_{3}\right) \\
& =-i \lambda \int \frac{d^{D} k_{1}}{(2 \pi)^{D}} \frac{d^{D} k_{2}}{(2 \pi)^{D}} \frac{d^{D} k_{3}}{(2 \pi)^{D}} \frac{d^{D} q_{1}}{(2 \pi)^{D}} \frac{d^{D} q_{2}}{(2 \pi)^{D}} \frac{d^{D} q_{3}}{(2 \pi)^{D}}(2 \pi)^{D} \delta^{D}\left(k_{1}+k_{2}+k_{3}\right) \cos \left(\frac{k_{1} \wedge k_{2}}{2}\right) \\
& \times \widetilde{\Delta}_{F}\left(q_{1}\right) \widetilde{\Delta}_{F}\left(q_{2}\right) \widetilde{\Delta}_{F}\left(q_{3}\right)(2 \pi)^{D} \delta^{D}\left(p_{1}-q_{1}\right)(2 \pi)^{D} \delta^{D}\left(p_{2}-q_{2}\right)(2 \pi)^{D} \delta^{D}\left(p_{3}-q_{3}\right) \\
& \times(2 \pi)^{D} \delta^{D}\left(k_{1}+q_{1}\right)(2 \pi)^{D} \delta^{D}\left(k_{2}+q_{2}\right)(2 \pi)^{D} \delta^{D}\left(k_{3}+q_{3}\right) \\
& =-i \lambda(2 \pi)^{D} \delta^{D}\left(p_{1}+p_{2}+p_{3}\right) \cos \left(\frac{p_{1} \wedge p_{2}}{2}\right) \widetilde{\Delta}_{F}\left(p_{1}\right) \widetilde{\Delta}_{F}\left(p_{2}\right) \widetilde{\Delta}_{F}\left(p_{3}\right) .
\end{aligned}
$$

O fator do vértice é obtido eliminando-se desse resultado os propagadores e também o fator global que inclui a delta de conservação de energia-momento, $(2 \pi)^{D} \delta\left(p_{1}+p_{2}+p_{3}\right)$. Finalmente, temos a seguinte expressão associada ao vértice,

$$
-i \lambda \cos \left(\frac{p_{1} \wedge p_{2}}{2}\right) \text {. }
$$

Lembramos que as partes quadráticas não são alteradas pelo produto Moyal. Logo, o propagador e as funções $\Delta^{ \pm}$são aquelas dadas no primeiro capítulo.

A relação de unitariedade que vamos investigar é mostrada na figura (2.1). De acordo com as regras de Feynman, a expressão associada ao diagrama do lado esquerdo é: 


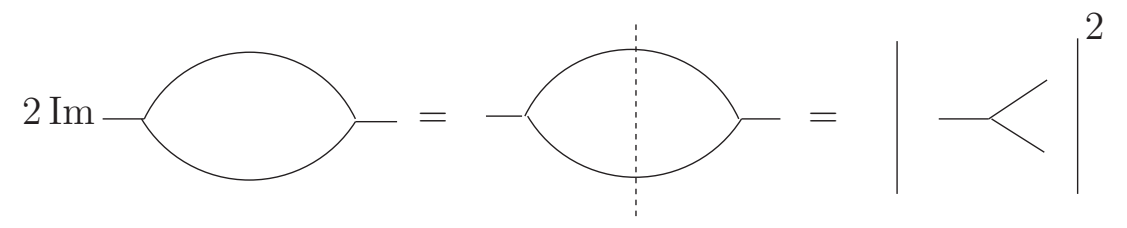

Figura 2.1: Regra de corte a um laço.

$$
\begin{aligned}
i T & =-\frac{\lambda^{2}}{2} \int \frac{d^{D} l}{(2 \pi)^{D}} \cos ^{2}\left(\frac{p \wedge l}{2}\right) \widetilde{\Delta}_{F}(l) \widetilde{\Delta}_{F}(l+p) \\
& =\frac{\lambda^{2}}{2} \int \frac{d^{D} l}{(2 \pi)^{D}} \cos ^{2}\left(\frac{p \wedge l}{2}\right) \frac{1}{l^{2}-m^{2}+i \epsilon} \frac{1}{(l+p)^{2}-m^{2}+i \epsilon}
\end{aligned}
$$

Por outro lado, a expressão do diagrama cortado é dada por,

$$
\begin{aligned}
& \sum_{|T|^{2}} \\
= & \frac{\lambda^{2}}{2} \int \frac{d^{D} k}{(2 \pi)^{D}} \cos ^{2}\left(\frac{k \wedge p}{2}\right) \widetilde{\Delta}^{+}(p-k) \widetilde{\Delta}^{+}(k) \\
= & \frac{\lambda^{2}}{2} \int \frac{d^{D} k}{(2 \pi)^{D}} \cos ^{2}\left(\frac{k \wedge p}{2}\right) 2 \pi \theta\left(p^{0}-k^{0}\right) \delta^{D}\left((p-k)^{2}-m^{2}\right) 2 \pi \theta\left(k^{0}\right) \delta^{D}\left(k^{2}-m^{2}\right) .
\end{aligned}
$$

Vale ressaltar que para o diagama cortado, no lugar do propagador $\widetilde{\Delta}_{F}$, aparece a função $\widetilde{\Delta}^{+}$. Para deixar essa equação numa forma mais conveniente, introduzimos a integral trivial

$$
\int d^{D} q \delta^{D}(q-p+k)=1 .
$$

Segue que

$$
\begin{aligned}
& \sum_{|T|^{2}} \\
= & \frac{\lambda^{2}}{2} \frac{1}{(2 \pi)^{D-2}} \int d^{D} k d^{D} q \cos ^{2}\left(\frac{k \wedge p}{2}\right) \theta\left(q^{0}\right) \delta^{D}\left(q^{2}-m^{2}\right) \theta\left(k^{0}\right) \delta^{D}\left(k^{2}-m^{2}\right) \delta^{D}(q-p+k) \\
= & \frac{\lambda^{2}}{2} \frac{1}{(2 \pi)^{D-2}} \int \frac{d^{D-1} k}{2 \omega_{k}} \frac{d^{D-1} q}{2 \omega_{q}} \cos ^{2}\left(\frac{k \wedge p}{2}\right) \delta^{D}(p-q-k),
\end{aligned}
$$

sendo que integramos sobre as componentes $k_{0}$ e $q_{0}$, fixando assim seus valores em $\omega_{k}=$ $\sqrt{\mathbf{k}^{2}+m^{2}}$ e $\omega_{q}=\sqrt{\mathbf{q}^{2}+m^{2}}$. 
Com o auxílio da identidade $\cos ^{2} x=(1+\cos 2 x) / 2$, as equações (2.6) e (2.9) podem ser separadas em duas partes. A parte sem o fator de co-seno é aquela que aparece no caso comutativo, não possuindo problemas com unitariedade. Então, temos que analisar apenas com a parte que contém o co-seno.

\subsubsection{Diagrama de um laço}

Vamos começar a trabalhar com a (2.6). De acordo com o que foi dito acima, a expressão que vamos considerar é dada por

$$
i T_{N C}=\frac{\lambda^{2}}{4} \int \frac{d^{D} l}{(2 \pi)^{D}} \cos (p \wedge l) \frac{1}{l^{2}-m^{2}+i \epsilon} \frac{1}{(l+p)^{2}-m^{2}+i \epsilon},
$$

com o subscrito $N C$ do lado esquerdo referindo-se a não comutativo. Primeiramente, vamos empregar a parametrização de Feynman

$$
\frac{1}{a b}=\int_{0}^{1} d x \frac{1}{[a x+b(1-x)]^{2}}
$$

para reagrupar o denominador. Identificando $a \equiv(l+p)^{2}-m^{2}+i \epsilon$ e $b \equiv l^{2}-m^{2}+i \epsilon$, podemos escrever o denominador como

$$
\begin{aligned}
\text { denominador } & =\left[\left((l+p)^{2}-m^{2}+i \epsilon\right) x+\left(l^{2}-m^{2}+i \epsilon\right)(1-x)\right]^{2} \\
& =\left[(l+p x)^{2}+p^{2} x(1-x)-m^{2}+i \epsilon\right]^{2},
\end{aligned}
$$

$\mathrm{Na}$ última igualdade simplesmente somamos e subtraímos o termo $p^{2} x^{2}$ para formar um quadrado completo. Com isso, (2.10) torna-se,

$$
i T_{N C}=\frac{\lambda^{2}}{4} \int_{0}^{1} d x \int \frac{d^{D} l}{(2 \pi)^{D}} \cos (p \wedge l) \frac{1}{\left[l^{2}+p^{2} x(1-x)-m^{2}+i \epsilon\right]^{2}},
$$

em que fizemos a mudança de variável $l \rightarrow l-p x$. A próxima etapa é empregar a parametrização de Schwinger:

$$
\frac{i}{k^{2}-m^{2}+i \epsilon}=\int_{0}^{\infty} d \alpha e^{i \alpha\left(k^{2}-m^{2}+i \epsilon\right)}
$$

Como o denominador do integrando de (2.13) está elevado ao quadrado, tomamos a derivada em relação à $m^{2}$ na equação acima, tal que

$$
\frac{1}{\left(k^{2}-m^{2}+i \epsilon\right)^{2}}=-\int_{0}^{\infty} d \alpha \alpha e^{i \alpha\left(k^{2}-m^{2}+i \epsilon\right)} .
$$

Identificando $k^{2}-m^{2}+i \epsilon \equiv l^{2}+p^{2} x(1-x)-m^{2}+i \epsilon$, escrevemos (2.13) como 
Unitariedade no modelo $\phi^{3}$ não comutativo

$$
i T_{N C}=-\frac{\lambda^{2}}{4} \int_{0}^{1} d x \int \frac{d^{D} l}{(2 \pi)^{D}} \cos (p \wedge l) \int_{0}^{\infty} d \alpha \alpha e^{i \alpha\left(l^{2}+p^{2} x(1-x)-m^{2}+i \epsilon\right)} .
$$

Expressando o cos $(p \wedge l)$ em termos de exponenciais imaginárias, de acordo com a identidade $\cos x=\left(e^{i x}+e^{-i x}\right) / 2$, e notando que cada exponencial fornece a mesma contribuição, obtemos o seguinte:

$$
i T_{N C}=-\frac{\lambda^{2}}{4} \int_{0}^{1} d x \int_{0}^{\infty} d \alpha \alpha e^{i \alpha\left(p^{2} x(1-x)-m^{2}+i \epsilon\right)} \int \frac{d^{D} l}{(2 \pi)^{D}} e^{i \alpha l^{2}+i p \wedge l} .
$$

A integral em $l$ é uma integral de Fresnel, que é uma generalização da gaussiana para o caso complexo. Para calculá-la, basta completar o quadrado no expoente:

$$
\begin{aligned}
\text { expoente } & =i \alpha\left[l_{\mu} l^{\mu}-\frac{1}{\alpha} l^{\mu} \Theta_{\mu \nu} p^{\nu}+\left(\frac{1}{2 \alpha} \Theta_{\mu \nu} p^{\nu}\right)^{2}-\left(\frac{1}{2 \alpha} \Theta_{\mu \nu} p^{\nu}\right)^{2}\right] \\
& =i \alpha\left(l_{\mu}-\frac{1}{2 \alpha} \Theta_{\mu \nu} p^{\nu}\right)^{2}+\frac{i}{4 \alpha} p \circ p
\end{aligned}
$$

em que definimos $p \circ p=p^{\mu} \Theta_{\mu \nu}^{2} p^{\nu}$. Fazendo $l_{\mu} \rightarrow l_{\mu}+\frac{1}{2 \alpha} \Theta_{\mu \nu} p^{\nu}$, a integral em $l$ torna-se

$$
\begin{aligned}
I_{F} & \equiv e^{\frac{i}{4 \alpha} p \circ p} \int \frac{d^{D} l}{(2 \pi)^{D}} e^{i \alpha l^{2}} \\
& =\frac{e^{\frac{i}{4 \alpha} p \circ p}}{(2 \pi)^{D}} \int d l_{0} e^{i \alpha l_{0}^{2}} \int d^{D-1} l e^{-i \alpha \mathbf{l}^{2}} \\
& =\frac{e^{\frac{i}{4 \alpha} p \circ p}}{(2 \pi)^{D}} \sqrt{\frac{i \pi}{\alpha}}\left(\sqrt{\frac{\pi}{i \alpha}}\right)^{D-1} .
\end{aligned}
$$

Substituindo em (2.17), obtemos

$$
i T_{N C}=-\frac{\lambda^{2}}{4} \frac{i}{(4 \pi)^{D / 2} i^{D / 2}} \int_{0}^{1} d x \int_{0}^{\infty} d \alpha \alpha^{1-D / 2} e^{i \alpha\left(p^{2} x(1-x)-m^{2}+i \epsilon\right)+\frac{i}{4 \alpha} p \circ p} .
$$

Vamos examinar essa expressão para o caso de três e quatro dimensões. Primeiramente, consideremos $D=3$. Segue que

$$
T_{N C}^{D=3}=-\frac{\lambda^{2}}{4} \frac{1}{(4 \pi)^{3 / 2} i^{3 / 2}} \int_{0}^{1} d x \int_{0}^{\infty} d \alpha \alpha^{-1 / 2} e^{i \alpha\left(p^{2} x(1-x)-m^{2}+i \epsilon\right)+\frac{i}{4 \alpha} p \circ p} .
$$


A integral em $\alpha$ pode ser avaliada usando o resultado:

$$
\int_{0}^{\infty} d y y^{-1 / 2} e^{i A y+\frac{i B}{y}}=\frac{e^{-2 \sqrt{-i A} \sqrt{-i B}} \sqrt{\pi}}{\sqrt{-i A}}, \text { se } \operatorname{Im} A>0 \text { e } \operatorname{Im} B>0 .
$$

Identificamos $A \equiv p^{2} x(1-x)-m^{2}+i \epsilon$ e $B \equiv \frac{p \circ p}{4}$. Notemos que o fator $A$ satisfaz a condição $\operatorname{Im} A>0$. Para o fator $B$, adicionamos a $\frac{p o p}{4}$ uma quantidade infinitesimal $i \delta$ e logo após o cálculo da integral aplicamos o limite $\delta \rightarrow 0$. Então,

$$
T_{N C}^{D=3}=-\frac{\lambda^{2}}{32 \pi} \frac{1}{i^{\frac{3}{2}}} \int_{0}^{1} d x \frac{e^{-\sqrt{-i p \circ p} \sqrt{-i\left(p^{2} x(1-x)-m^{2}+i \epsilon\right)}}}{\sqrt{-i\left(p^{2} x(1-x)-m^{2}+i \epsilon\right)}} .
$$

Na sequência, vamos analisar o caso $D=4$. Dessa forma,

$$
T_{N C}^{D=4}=\frac{\lambda^{2}}{4} \frac{1}{(4 \pi)^{2}} \int_{0}^{1} d x \int_{0}^{\infty} d \alpha \alpha^{-1} e^{i \alpha\left(p^{2} x(1-x)-m^{2}+i \epsilon\right)+\frac{i}{4 \alpha} p \circ p} .
$$

Novamente usaremos um resultado conhecido para calcular a integral em $\alpha$, ou seja,

$$
\int_{0}^{\infty} d y y^{-1} e^{i A y+\frac{i B}{y}}=2 K_{0}(2 \sqrt{-i A} \sqrt{-i B}), \text { se } \operatorname{Im} A>0 \text { e } \operatorname{Im} B>0,
$$

sendo $K_{0}$ a função de Bessel modificada de ordem zero. Fazendo as mesmas identificações que no caso anterior, segue diretamente

$$
T_{N C}^{D=4}=\frac{\lambda^{2}}{32 \pi^{2}} \int_{0}^{1} d x K_{0}\left(\sqrt{-i p \circ p} \sqrt{-i\left(p^{2} x(1-x)-m^{2}+i \epsilon\right)}\right) .
$$

Nossa tarefa seguinte é calcular a parte imaginária dessas amplitudes. Entretanto, neste ponto, devemos fazer algumas considerações. O cálculo da parte imaginária depende do sinal de $p \circ p$ e esse fato tem consequências muito importantes na análise da unitariedade. De acordo com a definição de $p \circ p$, seque que

$$
\begin{aligned}
p \circ p & \equiv p^{\mu} \Theta_{\mu \nu}^{2} p^{\nu} \\
& =\left(p_{0} \Theta^{0 i}\right)^{2}-2 p^{0} \Theta_{0 i} \Theta_{i j} p^{j}-\left(\Theta_{0 i} p^{i}\right)^{2}+\left(p^{i} \Theta_{i j}\right)^{2} .
\end{aligned}
$$

Se a não comutatividade envolve apenas o espaço, ou seja, se $\Theta_{0 i}=0$, temos,

$$
p \circ p=\left(p^{i} \Theta_{i j}\right)^{2}>0 .
$$

Nesse caso, $p \circ p$ é positivo definido. Por outro lado, no caso em que a não comutatividade envolve o espaço e o tempo, quer dizer, $\Theta_{0 i} \neq 0$ mas $\Theta_{i j}=0, p \circ p$ pode ser negativo. De fato, 


$$
p \circ p=\left(p_{0} \Theta^{0 i}\right)^{2}-\left(\Theta_{0 i} p^{i}\right)^{2} \geq\left(p_{0} \Theta^{0 i}\right)^{2}-\Theta_{0 i}^{2}\left(p^{i}\right)^{2}=p^{2} \Theta_{0 i}^{2} .
$$

Então, se $p^{2}<0$, podemos ter $p \circ p<0$. Essencialmente, temos dois casos de interesse a serem considerados: $p \circ p>0$ e $p^{2}>0$, caracterizando a não comutatividade apenas espacial; e $p \circ p<0$ e $p^{2}<0$ caracterizando a não comutatividade espaço-temporal.

\subsubsection{Não comutatividade espacial: $p \circ p>0$}

Para calcular a parte imaginária das amplitudes (2.23) e (2.26) devemos estudar o comportamento do termo $m^{2}-p^{2} x(1-x)$ no intervalo [0,1]. Para isto, definimos a função

$$
f(x) \equiv x^{2}-x+\frac{m^{2}}{p^{2}}
$$

Aqui, vamos considerar $p^{2}>4 m^{2} . f(x)$ é uma parábola com raízes $x=\frac{1 \pm \gamma}{2}$, sendo $\gamma \equiv$ $\sqrt{1-\frac{4 m^{2}}{p^{2}}}$. Nos intervalos $\left[0, \frac{1-\gamma}{2}\right]$ e $\left[\frac{1+\gamma}{2}, 1\right], f(x)$ é positiva. Em $\left[\frac{1-\gamma}{2}, \frac{1+\gamma}{2}\right], f(x)$ tornase negativa. Logo, na nossa análise, devemos considerar esses intervalos separadamente. Em primeiro lugar, vamos calcular a contribuição para a parte imaginária devido aos intervalos em que $f(x)$ é positiva. De acordo com a identidade $\operatorname{Im} z=\left(z-z^{*}\right) / 2 i$, temos

$$
\begin{aligned}
\operatorname{Im} T_{N C}^{D=3} & =\frac{1}{2 i} \frac{\lambda^{2}}{32 \pi} \int_{0}^{\frac{1-\gamma}{2}} d x\left(\frac{e^{-\sqrt{p \circ p\left(p^{2} f(x)-i \epsilon\right)}}}{\sqrt{\left(p^{2} f(x)-i \epsilon\right)}}-\frac{e^{-\sqrt{p \circ p\left(p^{2} f(x)+i \epsilon\right)}}}{\sqrt{\left(p^{2} f(x)+i \epsilon\right)}}\right) \\
& =0
\end{aligned}
$$

quando tomamos o limite $\epsilon \rightarrow 0$. Notemos que o mesmo resultado é válido para o intervalo $\left[\frac{1+\gamma}{2}, 1\right]$. Então, o único intervalo que contribui para a parte imaginária é $\left[\frac{1-\gamma}{2}, \frac{1+\gamma}{2}\right]$, no qual $f(x)<0$. A presença de fatores de $i$ com potências fracionárias requer cuidado especial com manipulações algébricas. Dessa maneira,

$$
T_{N C}^{D=3}=-\frac{\lambda^{2}}{32 \pi} \int_{\frac{1-\gamma}{2}}^{\frac{1+\gamma}{2}} d x \frac{e^{i \sqrt{p o p\left(p^{2}|f(x)|+i \epsilon\right)}}}{i \sqrt{\left(p^{2}|f(x)|+i \epsilon\right)}} .
$$

A parte imaginária é dada por 


$$
\begin{aligned}
\operatorname{Im} T_{N C}^{D=3} & =-\frac{1}{2 i} \frac{\lambda^{2}}{32 \pi} \int_{\frac{1-\gamma}{2}}^{\frac{1+\gamma}{2}} d x\left(\frac{e^{i \sqrt{p \circ p p^{2}|f(x)|}}}{i \sqrt{p^{2}|f(x)|}}-\frac{e^{-i \sqrt{p \circ p p^{2}|f(x)|}}}{-i \sqrt{p^{2}|f(x)|}}\right) \\
& =\frac{\lambda^{2}}{32 \pi} \int_{\frac{1-\gamma}{2}}^{\frac{1+\gamma}{2}} d x \frac{\cos \left(\sqrt{p \circ p p^{2}|f(x)|}\right)}{\sqrt{p^{2}|f(x)|}} \\
& =\frac{\lambda^{2}}{32 \pi} \int_{\frac{1-\gamma}{2}}^{\frac{1+\gamma}{2}} d x \frac{\cos \left(\sqrt{p \circ p p^{2}\left(-x^{2}+x-\frac{m^{2}}{p^{2}}\right)}\right)}{\sqrt{p^{2}\left(-x^{2}+x-\frac{m^{2}}{p^{2}}\right)}}
\end{aligned}
$$

sendo que na última igualdade apenas explicitamos a função $f(x)$ levando em conta o módulo. Agora, vamos ao cálculo da integral em $x$. Para esse fim, completamos o quadrado do termo

$$
\begin{aligned}
-x^{2}+x+\frac{m^{2}}{p^{2}} & =-\left[\left(x-\frac{1}{2}\right)^{2}+\frac{m^{2}}{p^{2}}-\frac{1}{4}\right] \\
& =\frac{\gamma^{2}}{4}-\left(x-\frac{1}{2}\right)^{2}
\end{aligned}
$$

e fazemos a mudança de variável $y \equiv x-\frac{1}{2}$. Assim,

$$
\operatorname{Im} T_{N C}^{D=3}=\frac{\lambda^{2}}{32 \pi} \int_{\frac{-\gamma}{2}}^{\frac{\gamma}{2}} d y \frac{\cos \left(\sqrt{p \circ p p^{2}} \frac{\gamma}{2}\left(1-\frac{y^{2}}{\gamma^{2} / 4}\right)^{\frac{1}{2}}\right)}{\sqrt{p^{2}} \frac{\gamma}{2}\left(1-\frac{y^{2}}{\gamma^{2} / 4}\right)^{\frac{1}{2}}} .
$$

Na sequência, fazemos outra mudança de variável, $t \equiv \frac{y}{\gamma / 2}$, conduzindo à

$$
\operatorname{Im} T_{N C}^{D=3}=\frac{\lambda^{2}}{32 \pi} \frac{1}{\sqrt{p^{2}}} \int_{-1}^{1} d t \frac{\cos \left(\sqrt{p \circ p p^{2}} \frac{\gamma}{2}\left(1-t^{2}\right)^{\frac{1}{2}}\right)}{\left(1-t^{2}\right)^{\frac{1}{2}}} .
$$

Essa expressão está pronta para ser comparada com uma representação integral da função de Bessel de ordem zero $J_{0}$, isto é,

$$
\int_{0}^{1} d x \frac{\cos (p x) \cosh \left(q \sqrt{1-x^{2}}\right)}{\sqrt{1-x^{2}}}=\frac{\pi}{2} J_{0}\left(\sqrt{p^{2}-q^{2}}\right) .
$$

Para deixá-la na mesma forma que (2.36), basta escolher $p=0$ e $q=i z(z>0)$. O resultado é

$$
\int_{-1}^{1} d x \frac{\cos \left(z \sqrt{1-x^{2}}\right)}{\sqrt{1-x^{2}}}=\pi J_{0}(z)
$$


em que usamos a propriedade $\cosh (i z)=\cos (z)$ e exploramos a paridade em $x$ do integrando para dobrar o intervalo de integração. Finalmente, identificamos $z \equiv \sqrt{p \circ p p^{2}} \frac{\gamma}{2}$, tal que

$$
\operatorname{Im} T_{N C}^{D=3}=\frac{\lambda^{2}}{32} \frac{1}{\sqrt{p^{2}}} J_{0}\left(\sqrt{p \circ p p^{2}} \frac{\gamma}{2}\right) .
$$

Esse resultado será comparado com a expressão correspondente ao diagrama cortado da figura (2.1).

Agora, vamos ao caso $D=4$. A análise aqui é essencialmente a mesma do caso anterior. Devemos considerar os intervalos $\left[0, \frac{1-\gamma}{2}\right],\left[\frac{1-\gamma}{2}, \frac{1+\gamma}{2}\right]$ e $\left[\frac{1+\gamma}{2}, 1\right]$ separadamente. No intervalo $\left[0, \frac{1-\gamma}{2}\right]$, em que $f(x)$ é positiva, temos

$$
T_{N C}^{D=4}=\frac{\lambda^{2}}{32 \pi^{2}} \int_{0}^{\frac{1-\gamma}{2}} d x K_{0}\left(\sqrt{p \circ p\left(p^{2} f(x)-i \epsilon\right)}\right) .
$$

Logo, no limite $\epsilon \rightarrow 0$, ficamos com $K_{0}\left(\sqrt{p \circ p p^{2} f(x)}\right)$ no integrando. Usando o fato que $K_{0}(x)$ é real quando $x$ é real, obtemos $\operatorname{Im} K_{0}=0$ nesse intervalo. O mesmo vale em $\left[\frac{1+\gamma}{2}, 1\right]$. Tal como no caso $D=3$, teremos contribuição para a parte imaginária apenas no intervalo $\left[\frac{1-\gamma}{2}, \frac{1+\gamma}{2}\right]$, ou seja,

$$
\operatorname{Im} T_{N C}^{D=4}=\frac{\lambda^{2}}{32 \pi^{2}} \int_{\frac{1-\gamma}{2}}^{\frac{1+\gamma}{2}} d x \operatorname{Im} K_{0}\left(-i \sqrt{p \circ p\left(p^{2}|f(x)|+i \epsilon\right)}\right) .
$$

De acordo com a propriedade $\operatorname{Im} K_{0}(-i x)=\frac{\pi}{2} J_{0}(x)$, podemos escrever

$$
\begin{aligned}
\operatorname{Im} T_{N C}^{D=4} & =\frac{\lambda^{2}}{64 \pi} \int_{\frac{1-\gamma}{2}}^{\frac{1+\gamma}{2}} d x J_{0}\left(\sqrt{p \circ p p^{2}|f(x)|}\right) \\
& =\frac{\lambda^{2}}{64 \pi} \int_{\frac{1-\gamma}{2}}^{\frac{1+\gamma}{2}} d x J_{0}\left(\sqrt{p \circ p p^{2}\left(-x^{2}+x-\frac{m^{2}}{p^{2}}\right)}\right) .
\end{aligned}
$$

Aqui, fazemos o mesmo procedimento que no caso $D=3$ : completamos o quadrado do termo $-x^{2}+x-\frac{m^{2}}{p^{2}}$ e em seguida a mudança de variável $y \equiv x-\frac{1}{2}$. Segue que

$$
\operatorname{Im} T_{N C}^{D=4}=\frac{\lambda^{2}}{64 \pi} \int_{\frac{-\gamma}{2}}^{\frac{\gamma}{2}} d y J_{0}\left(\sqrt{p \circ p p^{2}\left(\frac{\gamma^{2}}{4}-y^{2}\right)}\right) .
$$

Essa integral pode ser avaliada via

$$
\int_{0}^{z} d x J_{0}\left(\sqrt{z^{2}-x^{2}}\right)=\operatorname{sen} z
$$


Para comparar (2.43) com a expressão acima, a escrevemos como

$$
\begin{aligned}
\operatorname{Im} T_{N C}^{D=4} & =\frac{\lambda^{2}}{64 \pi}\left[\int_{\frac{-\gamma}{2}}^{0} d y J_{0}\left(\sqrt{p \circ p p^{2} \frac{\gamma^{2}}{4}-p \circ p p^{2} y^{2}}\right)\right. \\
& \left.+\int_{0}^{\frac{\gamma}{2}} d y J_{0}\left(\sqrt{p \circ p p^{2} \frac{\gamma^{2}}{4}-p \circ p p^{2} y^{2}}\right)\right]
\end{aligned}
$$

e fazemos outra mudança de variável, $x \equiv \sqrt{p \circ p p^{2}} y$, resultando em

$$
\begin{aligned}
\operatorname{Im} T_{N C}^{D=4} & =\frac{\lambda^{2}}{64 \pi}\left[-\int_{0}^{\frac{-\gamma}{2} \sqrt{p \circ p p^{2}}} d y J_{0}\left(\sqrt{p \circ p p^{2} \frac{\gamma^{2}}{4}-x^{2}}\right)\right. \\
& \left.+\int_{0}^{\frac{\gamma}{2} \sqrt{p \circ p p^{2}}} d y J_{0}\left(\sqrt{p \circ p p^{2} \frac{\gamma^{2}}{4}-x^{2}}\right)\right] .
\end{aligned}
$$

Finalmente, identificando $z \equiv \mp \frac{\gamma}{2} \sqrt{p \circ p p^{2}}$ na primeira e na segunda integral, respectivamente, obtemos

$$
\begin{aligned}
\operatorname{Im} T_{N C}^{D=4} & =\frac{\lambda^{2}}{64 \pi}\left[-\frac{\operatorname{sen}\left(-\frac{\gamma}{2} \sqrt{p \circ p p^{2}}\right)}{\sqrt{p \circ p p^{2}}}+\frac{\operatorname{sen}\left(\frac{\gamma}{2} \sqrt{p \circ p p^{2}}\right)}{\sqrt{p \circ p p^{2}}}\right] \\
& =\frac{\lambda^{2}}{32 \pi} \frac{\operatorname{sen}\left(\frac{\gamma}{2} \sqrt{p \circ p p^{2}}\right)}{\sqrt{p \circ p p^{2}}} .
\end{aligned}
$$

A seguir, vamos avaliar a expressão correspondente ao diagrama cortado, equação (2.9), em três e quatro dimensões para comparar com os resultados obtidos e verificar se satisfazem a relação de unitariedade.

\subsubsection{Diagrama cortado}

Para comparar com os resultados anteriores, primeiramente consideraremos o caso $p \circ p>0$ e $p^{2}>4 m^{2}$. Tomando $D=3$ em (2.9) e selecionando apenas a parte não comutativa de acordo com a identidade $\cos ^{2} x=(1+\cos 2 x) / 2$, ficamos com

$$
\sum|T|_{N C, D=3}^{2}=\frac{1}{2} \frac{\lambda^{2}}{2} \frac{1}{2 \pi} \int \frac{d^{2} k}{2 \omega_{k}} \frac{d^{2} q}{2 \omega_{q}} \cos (k \wedge p) \delta^{3}(p-q-k) .
$$


Para calcular essa integral, escolhemos o referencial do centro de momentos, tal que, $\mathbf{p}=0$. É importante observar que essa escolha preserva $p \circ p>0$. Dessa forma,

$$
\begin{aligned}
\sum|T|_{N C, D=3}^{2} & =\frac{\lambda^{2}}{2} \frac{1}{2 \pi} \int \frac{d^{2} k}{2 \omega_{k}} \frac{d^{2} q}{2 \omega_{q}} \cos (k \wedge p) \delta\left(p_{0}-q_{0}-k_{0}\right) \delta^{2}(\mathbf{q}+\mathbf{k}) \\
& =\frac{\lambda^{2}}{32 \pi} \int \frac{d^{2} k}{\omega_{k}^{2}} \cos (k \wedge p) \delta\left(p_{0}-2 \omega_{k}\right) .
\end{aligned}
$$

Podemos escrever o produto $k \wedge p$ da seguinte maneira: $k \wedge p=k^{i} \Theta_{i 0} p^{0} \equiv \mathbf{k} \cdot \tilde{\mathbf{p}}=$ $|\mathbf{k}||\tilde{\mathbf{p}}| \cos \theta$, em que definimos $\tilde{p}^{i} \equiv \Theta_{i 0} p^{0}$. Assim, em coordenadas polares, (2.49) fica

$$
\sum|T|_{N C, D=3}^{2}=\frac{\lambda^{2}}{32 \pi} \int_{0}^{\infty} \frac{d|\mathbf{k}||\mathbf{k}|}{\mathbf{k}^{2}+m^{2}} \delta\left(p_{0}-2 \omega_{k}\right) \int_{0}^{2 \pi} d \theta \cos (|\mathbf{k}||\tilde{\mathbf{p}}| \cos \theta)
$$

A integral em $\theta$ é essencialmente uma representação de $J_{0}$, a saber,

$$
J_{0}(x)=\frac{1}{2 \pi} \int_{0}^{2 \pi} d \theta \cos (x \cos \theta)
$$

e a integral em $|\mathbf{k}|$ pode ser calculada com a ajuda da propriedade (A.2), que permite-nos escrever a função delta como

$$
\delta\left(p_{0}-2 \omega_{k}\right)=\frac{\delta\left(|\mathbf{k}|-\frac{1}{2} \sqrt{p_{0}^{2}-4 m^{2}}\right)}{2 \sqrt{p_{0}^{2}-4 m^{2}} / \sqrt{p_{0}^{2}}},
$$

transformando-a em uma integral trivial. Com esses resultados, obtemos

$$
\sum|T|_{N C, D=3}^{2}=\frac{\lambda^{2}}{16} \frac{1}{\sqrt{p_{0}^{2}}} J_{0}\left(\frac{1}{2} \sqrt{1-\frac{4 m^{2}}{p_{0}^{2}}} \sqrt{p_{0}^{2}}|\tilde{\mathbf{p}}|\right)
$$

Notemos que $|\tilde{\mathbf{p}}|=\sqrt{\tilde{\mathbf{p}} \cdot \tilde{\mathbf{p}}}=\sqrt{\Theta_{i 0} p^{0} \Theta_{i 0} p^{0}}=\sqrt{p^{0} \Theta_{0 i} \Theta^{i}{ }_{0} p^{0}}$, ou seja, o escrevemos numa forma covariante. Então, esse resultado deve ter a mesma forma em qualquer referencial, tal que podemos retomar o quadrimomento $p$ apenas trocando $p^{0}$ por $p$. Entretanto, para um $p$ qualquer (mas, $p^{2}>4 m^{2}$ ), a condição $p \circ p>0$ só é satisfeita se $\Theta_{0 i}=0$, ou seja, se a não comutatividade envolve apenas o espaço. Nesse sentido, (2.53) dá lugar a

$$
\sum|T|_{N C, D=3}^{2}=\frac{\lambda^{2}}{16} \frac{1}{\sqrt{p^{2}}} J_{0}\left(\frac{\gamma}{2} \sqrt{p^{2} p \circ p}\right),
$$

porém com $\Theta_{0 i}=0$. Assim, esse caso satisfaz a relação de unitariedade mostrada na figura (2.1).

Examinemos o caso $D=4$. Então, de (2.9), segue 


$$
\sum|T|_{N C, D=4}^{2}=\frac{1}{2} \frac{\lambda^{2}}{2} \frac{1}{(2 \pi)^{2}} \int \frac{d^{3} k}{2 \omega_{k}} \frac{d^{3} q}{2 \omega_{q}} \cos (k \wedge p) \delta^{4}(p-q-k) .
$$

O procedimento para calcular essas integrais é exatamente o mesmo que o do caso $D=3$. Assim, escolhemos o referencial do centro de momentos, $\mathbf{p}=0$. A integral em $\mathbf{q}$ é calculada usando a função delta $\delta^{3}(\mathbf{q}+\mathbf{k})$. Em seguida, escrevemos o elemento de volume de $\mathbf{k}$ em coordenadas esféricas, $d^{3} k=d|\mathbf{k}||\mathbf{k}|^{2} d \theta \operatorname{sen} \theta d \phi$. Para a integral em $|\mathbf{k}|$ usamos a função delta remanescente $\delta\left(p_{0}-q_{0}-k_{0}\right)$. A integral em $\phi$ é trivial. Por último, definimos o produto escalar $k \wedge p \equiv \mathbf{k} \cdot \tilde{\mathbf{p}}=|\mathbf{k}||\tilde{\mathbf{p}}| \cos \theta$ que conduz a integração em $\theta$. No final, retomamos o quadrimomento $p$ sob a imposição que $\Theta_{0 i}=0$. O resultado obtido é

$$
\sum|T|_{N C, D=4}^{2}=\frac{\lambda^{2}}{16 \pi} \frac{\operatorname{sen}\left(\frac{\gamma}{2} \sqrt{p^{2} p \circ p}\right)}{\sqrt{p^{2} p \circ p}},
$$

com $\Theta_{0 i}=0$. Logo, esse caso também satisfaz a relação de unitariedade. Em suma, para o caso $p \circ p>0$ a relação de unitariedade entre os diagramas considerados é satisfeita. A seguir, vamos investigar o caso em que $p \circ p<0$.

\subsubsection{Não comutatividade espaço-temporal: $p \circ p<0$}

Essa condição pode apenas ser obtida na presença de um momento tipo espaço, $p^{2}<0$. Sob as duas condições estabelecidas acima, devemos extrair a parte imaginária da equação (2.23) no caso em que $D=3$. Esse processo requer a análise da função $g(x) \equiv m^{2}-$ $p^{2} x(1-x)=m^{2}+\left|p^{2}\right| x(1-x)$. No intervalo entre 0 e 1 essa função é positiva. Assim, (2.23) torna-se

$$
T_{N C}^{D=3}=\frac{\lambda^{2}}{32 \pi} \int_{0}^{1} d x \frac{e^{-i \sqrt{|p \circ p|\left(m^{2}+\left|p^{2}\right| x(1-x)-i \epsilon\right)}}}{\sqrt{m^{2}+\left|p^{2}\right| x(1-x)-i \epsilon}} .
$$

A parte imaginária de $T_{N \bar{C}}^{D=3}$ é dada por

$$
\operatorname{Im} T_{N C}^{D=3}=-\frac{\lambda^{2}}{32 \pi} \int_{0}^{1} d x \frac{\operatorname{sen}\left(\sqrt{|p \circ p|\left(m^{2}+\left|p^{2}\right| x(1-x)\right)}\right)}{\sqrt{m^{2}+\left|p^{2}\right| x(1-x)}} .
$$

No caso de $D=4$, devemos considerar a equação (2.26). A mesma análise da função $g(x)$ definida acima é válida aqui. Então,

$$
T_{N C}^{D=4}=\frac{\lambda^{2}}{32 \pi^{2}} \int_{0}^{1} d x K_{0}\left(i \sqrt{|p \circ p|\left(m^{2}+\left|p^{2}\right| x(1-x)-i \epsilon\right)}\right) .
$$

Usando a propriedade $\operatorname{Im} K_{0}(i x)=\frac{\pi}{2} J_{0}(-x)=\frac{\pi}{2} J_{0}(x)$, obtemos 


$$
\operatorname{Im} T_{N C}^{D=4}=\frac{\lambda^{2}}{64 \pi} \int_{0}^{1} d x J_{0}\left(\sqrt{|p \circ p|\left(m^{2}+\left|p^{2}\right| x(1-x)\right.}\right) .
$$

As expressões (2.58) e (2.60) são claramente não nulas. Por outro lado, devido a presença da função delta de conservação $\delta^{D}(p-q-k)$ na expressão associada ao diagrama cortado, equação (2.9), quando $p^{2}<0$ essa expressão é nula (independentemente da dimensão considerada), pois com essa condição a função delta não pode ser satisfeita. Na verdade, isso reflete o fato que uma partícula com momento do tipo espaço não pode decair em duas partículas massivas, pois violaria a conservação de energia-momento. Portanto, há uma violação da unitariedade para o caso $\Theta_{0 i} \neq 0$, ou seja, quando a não comutatividade envolve o espaço e o tempo.

Para concluir este capítulo, discutiremos um pouco sobre essa quebra de unitariedade. Para esse fim, examinaremos o cálculo do fator de vértice, pois ele permite-nos localizar o problema. A contribuição perturbativa de ordem mais baixa não trivial para a função de três pontos é dada na equação (2.2). Dentro do esquema perturbativo usual, o ordenamento temporal é entendido sendo realizado antes da integração sobre o espaço-tempo nessa equação, tal que empregamos o teorema de Wick para escrever o produto dos campos ordenados no tempo em termos de propagadores $\Delta_{F}$. No procedimento adotado, a integral em $x$ foi usada para escrever o produto Moyal em termos do produto ordinário dos campos de acordo com o resultado (1.45). No caso de interações locais, isso não traria problemas. Entretanto, devido à não localidade no tempo do produto Moyal, esse procedimento mostra-se inadequado. Para ver precisamente isso, vamos calcular novamente o fator de vértice, porém, de uma maneira diferente. Inicialmente, escrevemos a lagrangiana de interação como

$$
\begin{aligned}
\mathcal{L}_{I} & =\frac{-i \lambda}{3 !} \int d^{D} x \frac{1}{2}[\phi(x) * \phi(x) * \phi(x)+\text { c.h. }] \\
& =\left.\frac{-i \lambda}{3 !} \int d^{D} x \cos \left[\frac{1}{2}\left[\partial_{x_{1}} \wedge\left(\partial_{x_{2}}+\partial_{x_{3}}\right)+\partial_{x_{2}} \wedge \partial_{x_{3}}\right]\right] \phi\left(x_{1}\right) \phi\left(x_{2}\right) \phi\left(x_{3}\right)\right|_{x_{i}=x} .
\end{aligned}
$$

Dessa forma, a equação (2.2) torna-se,

$$
\begin{gathered}
V_{3}=\frac{-i \lambda}{3 !} \int d^{D} x \\
\times\left\langle 0\left|T \phi\left(x_{1}\right) \phi\left(x_{2}\right) \phi\left(x_{3}\right) \cos \left[\frac{1}{2}\left[\partial_{y_{1}} \wedge\left(\partial_{y_{2}}+\partial_{y_{3}}\right)+\partial_{y_{2}} \wedge \partial_{y_{3}}\right]\right] \phi\left(y_{1}\right) \phi\left(y_{2}\right) \phi\left(y_{3}\right)\right| 0\right\rangle .
\end{gathered}
$$

Essa expressão fornecerá o mesmo resultado que (2.4) quando escrevemos as derivadas do produto Moyal à esquerda do ordenamento temporal, ou seja, 


$$
\begin{gathered}
V_{3}=\frac{-i \lambda}{3 !} \int d^{D} x \\
\times \quad \cos \left[\frac{1}{2}\left[\partial_{y_{1}} \wedge\left(\partial_{y_{2}}+\partial_{y_{3}}\right)+\partial_{y_{2}} \wedge \partial_{y_{3}}\right]\right]\left\langle 0\left|T \phi\left(x_{1}\right) \phi\left(x_{2}\right) \phi\left(x_{3}\right) \phi\left(y_{1}\right) \phi\left(y_{2}\right) \phi\left(y_{3}\right)\right| 0\right\rangle .
\end{gathered}
$$

Calculando as contrações de acordo com o teorema de Wick e tomando as transformadas de Fourier tal como no cálculo efetuado no início do capítulo, obtemos exatamente (2.4):

$$
\widetilde{V}_{3}=-i \lambda(2 \pi)^{D} \delta^{D}\left(p_{1}+p_{2}+p_{3}\right) \cos \left(\frac{p_{1} \wedge p_{2}}{2}\right) \widetilde{\Delta}_{F}\left(p_{1}\right) \widetilde{\Delta}_{F}\left(p_{2}\right) \widetilde{\Delta}_{F}\left(p_{3}\right)
$$

No entanto, quando trocamos a ordem entre o produto Moyal e o ordenamento temporal na equação (2.63) estamos cometendo um erro, pois o ordenamento temporal é realizado por meio de funções $\theta$ e existem derivadas temporais não polinomiais no produto Moyal quando $\Theta_{0 i} \neq 0$. Portanto, a expressão (2.64) não está correta, mostrando que as próprias regras de Feynman usuais não se aplicam quando $\Theta_{0 i} \neq 0$. Incluem-se aí as expressões (2.6) e (2.7) associadas aos diagramas da figura (2.1), que também não são corretas quando a não comutatividade envolve o espaço e o tempo. Essa é justamente a origem da quebra da unitariedade.

Por outro lado, a operação de troca entre o produto Moyal e o ordenamento temporal é permitida quando $\Theta_{0 i}=0$, pois no produto Moyal não haverá derivadas temporais. O resultado disso é que a unitariedade é preservada nesse caso. 


\section{Capítulo 3}

\section{Perturbação para Teorias Não Comutativas}

O objetivo deste capítulo é apresentar um método perturbativo que não tenha problemas com a quebra da unitariedade. Isso é feito partindo de uma matriz $S$ convenientemente definida para o caso teorias não comutativas. Definida a matriz $S$, fazemos algumas verificações que reforçam sua consistência.

\subsection{Algumas Definições}

Para iniciar nossa discussão, vamos considerar uma teoria escalar com interação do tipo

$$
\phi_{*}^{p} \equiv \phi * \phi * \cdots * \phi
$$

A langrangiana de interação num espaço com $D-1$ dimensões espaciais é dada por

$$
L_{I}(t)=\int d^{D-1} x \mathcal{L}_{I}\left(\phi_{*}(x)\right),
$$

com

$$
\mathcal{L}_{I}\left(\phi_{*}(x)\right)=-\frac{g}{p !} \phi_{*}^{p}
$$

Por conveniência, introduzimos o operador $\mathcal{F}_{x}$, definido por

$$
\mathcal{F}_{x}\left(\phi^{p}(x)\right) \equiv \phi(x) *^{x} \phi(x) *^{x} \cdots *^{x} \phi(x)=\phi_{*}^{p}(x)
$$


A notação $*^{x}$ é para explicitar que o produto Moyal está sendo tomado em relação à $x$. A densidade de lagrangiana de interação $\mathcal{L}_{I}\left(\phi_{*}(x)\right)$ está relacionada com o operador $\mathcal{F}_{x}$ por meio de

$$
\mathcal{L}_{I}\left(\phi_{*}(x)\right)=\mathcal{F}_{x}(\mathcal{V}(\phi(x)))
$$

em que $\mathcal{V}(\phi(x))$ é a densidade de lagrangiana de interação com produto ordinário entre os campos,

$$
\mathcal{V}(\phi(x)) \equiv-\frac{g}{p !} \phi^{p}(x)
$$

Com o operador $\mathcal{F}$, podemos escrever, por exemplo,

$$
A(x) * B(x)=\mathcal{F}_{x}(A(x) B(x)) .
$$

É importante observar que mesmo que $A(x) B(x)=B(x) A(x)$,

$$
\mathcal{F}_{x}(A(x) B(x)) \neq \mathcal{F}_{x}(B(x) A(x)) .
$$

Podemos definir também uma composição de operadores $\mathcal{F}$ da seguinte forma:

$$
\mathcal{F}_{x y} \equiv \mathcal{F}_{x} \mathcal{F}_{y}
$$

que é comutativo, quer dizer,

$$
\mathcal{F}_{x} \mathcal{F}_{y}=\mathcal{F}_{y} \mathcal{F}_{x}
$$

\subsection{Campos Assintóticos}

Após as consideraçẽs feitas acima, passemos à equação de Klein-Gordon na presença de uma fonte,

$$
\left(\partial^{2}+m^{2}\right) \phi(x)=\xi_{*}(\phi(x)),
$$

sendo que $\xi_{*}(\phi(x))$ é um funcional dos campos, obtido a partir de

$$
\begin{aligned}
\xi_{*}(\phi(x)) \equiv & \frac{\delta}{\delta \phi(x)} \int d t L_{I}(t) \\
= & -\frac{g}{p !} \int d^{D} y\left(\delta^{D}(x-y) *^{y} \phi_{*}^{p-1}(y)+\phi(y) *^{y} \delta^{D}(x-y) *^{y} \phi_{*}^{p-2}(y)\right. \\
& \left.+\cdots+\phi_{*}^{p-2}(y) *^{y} \delta^{D}(x-y) *^{y} \phi(y)+\phi_{*}^{p-1}(y) *^{y} \delta(x-y)\right) .
\end{aligned}
$$


Aqui, introduzimos a seguinte notação para a simetrização de $n$ quantidades distintas:

$$
\left\{\prod_{i=1}^{n} A_{i}\right\}_{s} \equiv \sum_{s(1,2, \cdots, n)} \prod_{i=1}^{n} A_{s(i)}
$$

com $s(1,2, \cdots, n)$ indicando as permutações de $1,2, \cdots, n$. Desse modo, podemos reescrever (3.12) como segue;

$$
\xi_{*}(\phi(x))=-\frac{g}{p !} \int d^{D} y \mathcal{F}_{y}\left\{\delta(x-y) \phi^{p-1}(y)\right\}_{s(y)},
$$

em que $s(y)$ refere-se a simetrização dos operadores com argumento $y$. A solução da equação (3.11) pode ser dada em termos das funções de Green retardada e avançada,

$$
\begin{aligned}
\phi(x) & =\phi_{i n}(x)+\int d^{D} y \Delta_{r e t}(x-y) \xi_{*}(\phi(y)) \\
& =\phi_{e m}(x)+\int d^{D} y \Delta_{a v}(x-y) \xi_{*}(\phi(y)) .
\end{aligned}
$$

Os campos assintóticos incidente e emergente, $\phi_{i n}$ e $\phi_{e m}$, são soluções da equação de Klein-Gordon homogênea. As funções $\Delta_{r e t}$ e $\Delta_{a v}$ são dadas por

$$
\Delta_{r e t}(x)=-\theta\left(x^{0}\right) \Delta(x)
$$

e

$$
\Delta_{a v}(x)=\theta\left(-x^{0}\right) \Delta(x)
$$

A função $\Delta(x)$ é definida como

$$
\left[\phi_{i n}(x), \phi_{i n}(0)\right]=\left[\phi_{e m}(x), \phi_{e m}(0)\right]=i \Delta(x) .
$$

Consideremos a primeira linha de (3.15). Explicitando a fonte $\xi_{*}$, temos,

$$
\begin{aligned}
\phi(x) & =\phi_{i n}(x)-\frac{g}{p !} \int d^{D} y \Delta_{r e t}(x-y) \int d^{D} z \mathcal{F}_{z}\left\{\delta(y-z) \phi^{p-1}(z)\right\}_{s(z)} \\
& =\phi_{i n}(x)+\int d^{D} y \mathcal{F}_{y}\left\{-\frac{g}{p !} \Delta_{r e t}(x-y) \phi^{p-1}(y)\right\}_{s(y)}
\end{aligned}
$$

Na última linha de (3.19) empregamos a identidade

$$
\int d^{D} y \int d^{D} z A(x-y)\left[\delta(y-z) *^{z} B(z)\right]=\int d^{D} y A(x-y) *^{y} B(y) .
$$


Nessa igualdade uamos o fato que partes quadráticas, quando integradas, não são alteradas pelo produto Moyal. Da mesma maneira,

$$
\phi(x)=\phi_{e m}(x)+\int d^{D} y \mathcal{F}_{y}\left\{-\frac{g}{p !} \Delta_{a v}(x-y) \phi^{p-1}(y)\right\}_{s(y)} .
$$

Podemos relacionar os campos $\phi_{i n}$ e $\phi_{\text {em }}$ a partir de (3.19) e (3.21), subtraíndo uma da outra. Desse modo,

$$
\begin{aligned}
\phi_{e m}(x) & =\phi_{i n}(x)+\int d^{D} y \mathcal{F}_{y}\left\{-\frac{g}{p !}\left[\Delta_{r e t}(x-y)-\Delta_{a v}(x-y)\right] \phi^{p-1}(y)\right\}_{s(y)} \\
& =\phi_{i n}(x)+\int d^{D} y \mathcal{F}_{y}\left\{\frac{g}{p !} \Delta(x-y) \phi^{p-1}(y)\right\}_{s(y)}
\end{aligned}
$$

em que usamos (3.16) e (3.17) para escrever $\Delta_{a v}$ e $\Delta_{r e t}$ em termos de $\Delta(x-y)$. A equação (3.22) é conhecida como equação de Yang-Feldman. Podemos usar as equações (3.19), (3.21) e (3.22) para escrever soluções iterativas tanto para o campo em interação $\phi$ como para o campo livre $\phi_{e m}$. Primeiramente, expressamos o campo $\phi$ por meio da série

$$
\phi=\sum_{n=0}^{\infty} \phi_{n}
$$

com $\phi_{n}$ designando um termo de ordem $g^{n}$. De acordo com (3.19), vemos que os primeiros termos são dados por

$$
\begin{gathered}
\phi_{0}(x)=\phi_{\text {in }}(x), \\
\phi_{1}(x)=\int d^{D} y \mathcal{F}_{y}\left\{-\frac{g}{p !} \Delta_{r e t}(x-y) \phi_{0}^{p-1}(y)\right\}_{s(y)} \\
\phi_{2}(x)=\int d^{D} y \mathcal{F}_{y}\left\{-\frac{g}{p !} \Delta_{r e t}(x-y) \phi_{0}^{p-2}(y) \phi_{1}(y)\right\}_{s(y)}, \\
\phi_{3}(x)=\int d^{D} y \mathcal{F}_{y}\left\{-\frac{g}{p !} \Delta_{r e t}(x-y)\left(\phi_{0}^{p-2}(y) \phi_{2}(y)+\phi_{0}^{p-3}(y) \phi_{1}^{2}(y)\right)\right\}_{s(y)} .
\end{gathered}
$$

Dessas formas explícitas podemos fazer a generalização para um termo de ordem $n$,

$$
\phi_{n}(x)=\int d^{D} y \mathcal{F}_{y}\left\{-\frac{g}{p !} \sum_{q_{1}+q_{2}+\cdots+q_{p-1}=n-1} \Delta_{r e t}(x-y) \phi_{q_{1}}(y) \cdots \phi_{q_{p-1}}(y)\right\}_{s(y)} .
$$


O mesmo procedimento para o campo assintótico $\phi_{\text {em }}$ conduz à,

$$
\phi_{e m}=\sum_{n=0}^{\infty} \varphi_{n}
$$

$\operatorname{com} \varphi_{0}=\phi_{0}$ e para $n \geq 1$

$$
\varphi_{n}(x)=\int d^{D} y \mathcal{F}_{y}\left\{\frac{g}{p !} \sum_{q_{1}+q_{2}+\cdots+q_{p-1}=n-1} \Delta(x-y) \phi_{q_{1}}(y) \cdots \phi_{q_{p-1}}(y)\right\}_{s(y)}
$$

\subsection{Matriz $S$}

A matriz $S$ conecta os campos $\phi_{\text {em }}$ e $\phi_{i n}$ de acordo com

$$
\phi_{e m}=S^{\dagger} \phi_{i n} S
$$

Escrevendo $S \equiv e^{i \delta}$, obtemos,

$$
\phi_{e m}=\phi_{i n}+\left[\phi_{i n}, i \delta\right]+\frac{1}{2}\left[\left[\phi_{i n}, i \delta\right], i \delta\right]+\cdots .
$$

Comparando com a equação (3.29), segue que,

$$
\begin{aligned}
{\left[\phi_{0}(x), i \delta\right] } & =\varphi_{1}(x)=\int d^{D} y \mathcal{F}_{y}\left\{\frac{g}{p !} \Delta(x-y) \phi_{0}^{p-1}(y)\right\}_{s(y)} \\
& =\int d^{D} y \mathcal{F}_{y}\left\{-\frac{g}{p !} i\left[\phi_{0}(x), \phi_{0}(y)\right] \phi_{0}^{p-1}(y)\right\}_{s(y)} \\
& =-i \frac{g}{p !} \int d^{D} y\left(\left[\phi_{0}(x), \phi_{0}(y)\right] *^{y} \phi_{0 *}^{p-1}(y)\right. \\
& \left.+\phi_{0 *}(y) *^{y}\left[\phi_{0}(x), \phi_{0}(y)\right] *^{y} \phi_{0 *}^{p-1}(y)+\cdots+\phi_{0 *}^{p-1}(y) *^{y}\left[\phi_{0}(x), \phi_{0}(y)\right]\right) \\
& =-i \frac{g}{p !} \int d^{D} y\left[\phi_{0}(x), \phi_{0 *}^{p}(y)\right] .
\end{aligned}
$$

Daí, obtemos $\delta$ em ordem $g$, ou seja,

$$
\begin{aligned}
\delta & =\int d^{D} y \mathcal{F}_{y}\left(-\frac{g}{p !} \phi_{0}^{p}(y)\right)+\mathcal{O}\left(g^{2}\right) \\
& =\int d^{D} y \mathcal{L}_{I}\left(\phi_{0 *}(y)\right)+\mathcal{O}\left(g^{2}\right)
\end{aligned}
$$


Esse resultado sugere a forma da matriz $S$. Levando em conta que ordens mais altas requerem ordenamento temporal, definimos a seguinte matriz $S$,

$$
S \equiv \sum_{n=0}^{\infty} i^{n} A_{n},
$$

sendo $A_{n}$ da ordem $g^{n}$, com $A_{0}=1$ e para $n \geq 1$,

$$
A_{n}=\iint \cdots \int d^{D} x_{1} d^{D} x_{2} \cdots d^{D} x_{n} \mathcal{F}_{12 \cdots n}\left[\theta_{12 \cdots n} \mathcal{V}\left(\phi_{0}\left(x_{1}\right)\right) \cdots \mathcal{V}\left(\phi_{0}\left(x_{n}\right)\right)\right]
$$

Na expressão acima, empregamos a composição definida em (3.10):

$$
\mathcal{F}_{12 \cdots n} \equiv \mathcal{F}_{x_{1}} \mathcal{F}_{x_{2}} \cdots \mathcal{F}_{x_{n}}
$$

A função $\theta_{12 \cdots n}$ é definida como segue,

$$
\theta_{12 \cdots n} \equiv \theta\left(t_{1}-t_{2}\right) \theta\left(t_{2}-t_{3}\right) \cdots \theta\left(t_{n-1}-t_{n}\right) .
$$

Neste ponto, temos que dar um significado preciso para a equação (3.36), pois ainda não é claro como se dá a atuação do operador $\mathcal{F}_{12 \cdots n}$ sobre $\theta_{12 \cdots n}$ e sobre $\mathcal{V}\left(\phi_{0}\left(x_{1}\right)\right) \cdots \mathcal{V}\left(\phi_{0}\left(x_{n}\right)\right)$. Uma maneira conveniente para fixar essa operação é dada no que segue. Para isso, consideremos o caso $n=2$ em (3.36). Então, adotamos a seguinte prescrição:

$$
\begin{aligned}
& \mathcal{F}_{x y}\left[\theta\left(x^{0}-y^{0}\right) \phi_{0}^{p}(x) \phi_{0}^{p}(y)\right] \\
& \rightarrow \mathcal{F}_{x y}\left[\left.\theta\left(x_{i}^{0}-y_{j}^{0}\right) \phi_{0}\left(x_{1}\right) \cdots \phi_{0}\left(x_{i}\right) \cdots \phi_{0}\left(x_{p}\right) \phi_{0}\left(y_{1}\right) \cdots \phi_{0}\left(y_{j}\right) \cdots \phi_{0}\left(y_{p}\right)\right|_{x_{i}=x, y_{j}=y}\right]
\end{aligned}
$$

se os campos $\phi_{0}\left(x_{i}\right)$ e $\phi_{0}\left(x_{j}\right)$ estão contraídos entre si. Dito de outra forma, a função $\theta\left(x^{0}-y^{0}\right)$ será usada para fazer o ordenamento temporal apenas entre dois campos: um campo com argumento $x$ e um com argumento $y$. Isso significa que usamos a função $\theta$ apenas uma vez. É importante esclarecer que o produto entre a função $\theta$ e os dois campos contraídos é um produto ordinário, não um produto Moyal. Esse procedimento é denominado realização minimal da função $\theta$. Mesmo na presença de muitas funções $\Delta(x-y)$ (ou $\Delta^{+}(x-y)$ ou $\Delta^{-}(x-y)$ ou outra função também obtida a partir da contração de dois campos), temos somente uma função $\theta$, quer dizer,

$$
\theta\left(x^{0}-y^{0}\right) \prod_{a, b} \Delta\left(x_{a}-y_{b}\right) \rightarrow \theta\left(x_{i}^{0}-y_{j}^{0}\right) \prod_{a, b} \Delta\left(x_{a}-y_{b}\right),
$$

em que $i(j)$ é um entre os valores possíveis de $a(b)$. Introduzindo o ordenamento temporalMoyal $T_{*}$ como 


$$
T_{*}\left[A\left(x_{1}^{0}\right) A\left(x_{2}^{0}\right)\right]=\mathcal{F}_{12}\left[\theta_{12} A\left(x_{1}^{0}\right) A\left(x_{2}^{0}\right)+\theta_{21} A\left(x_{2}^{0}\right) A\left(x_{1}^{0}\right)\right]
$$

podemos escrever a matriz $S$ da seguinte forma:

$$
\begin{aligned}
S & =\sum_{n=0}^{\infty} \frac{i^{n}}{n !} \int d^{D} x_{1} \cdots \int d^{D} x_{n} T_{*}\left[\mathcal{V}\left(\phi_{0}\left(x_{1}\right)\right) \cdots \mathcal{V}\left(\phi_{0}\left(x_{n}\right)\right)\right] \\
& \equiv T_{*} \exp \left[i \int d^{D} x \mathcal{V}\left(\phi_{0}(x)\right)\right] .
\end{aligned}
$$

Para estabelecer a consistência da matriz $S$ proposta acima, devemos fazer algumas verificações: Primeiro, devemos verificar que ela conecta os campos $\phi_{i n}$ e $\phi_{e m}$ corretamente, originando a série (3.29). Segundo, que ela é unitaria. Por fim, podemos checar a unitariedade com cálculos explícitos de diagramas em modelos específicos. Essa última verifição será deixada para os capítulos seguintes. Todas as verificações serão feitas perturbativamente.

\subsection{Matriz $S$ e Campos $\phi_{i n}$ e $\phi_{e m}$}

Nesta seção, vamos verificar que a matriz $S$ definida acima reproduz corretamente a série (3.29), via relação (3.31). Inicialmente, definimos $\Phi_{e m}$ tal que

$$
\Phi_{\text {em }}(x) \equiv S^{\dagger} \phi_{i n}(x) S=\sum_{n=0}^{\infty} \Phi_{(n)}(x)
$$

Substituindo (3.35) nessa expressão, obtemos

$$
\Phi_{(n)}(x)=\sum_{\substack{l, m \\ l+m=n}}(-1)^{l} i^{l+m} A_{l}^{\dagger} \phi_{0}(x) A_{m} .
$$

Notemos que $\Phi_{(n)}$ é da ordem $g^{n}$. Uma observação importante é que a operação $\dagger$ em $A_{n}^{\dagger}$ é aplicada apenas aos campos, não sobre $\mathcal{F}_{12 \cdots n}$ ou $\theta_{12 \cdots n}$. O procedimento seguinte é calcular $\Phi_{(n)}$, ordem por ordem, e comparar com (3.29). Conduziremos nossos cálculos até a ordem $g^{3}$.

Em ordem $g$, temos

$$
\begin{aligned}
\Phi_{(1)}(x) & =i\left(\phi_{0}(x) A_{1}-A_{1}^{\dagger} \phi_{0}(x)\right) \\
& =i \int d^{D} y \mathcal{F}_{y}\left(\left[\phi_{0}(x), \mathcal{V}\left(\phi_{0}(y)\right)\right]\right) .
\end{aligned}
$$


Vamos trabalhar apenas com o comutador, ou seja,

$$
\begin{aligned}
& {\left[\phi_{0}(x), \phi_{0}^{p}(y)\right] } \\
= & {\left[\phi_{0}(x), \phi_{0}(y)\right] \phi_{0}^{p-1}(y)+\phi_{0}(y)\left[\phi_{0}(x), \phi_{0}(y)\right] \phi_{0}^{p-2}(y)+\cdots+\phi_{0}^{p-1}(y)\left[\phi_{0}(x), \phi_{0}(y)\right] } \\
= & i\left\{\Delta(x-y) \phi_{0}^{p-1}(y)\right\}_{s(y)},
\end{aligned}
$$

em que usamos (3.18) para identificar a função $\Delta$. Dessa forma, chegamos ao seguinte resultado:

$$
\Phi_{(1)}(x)=\frac{g}{p !} \int d^{D} y \mathcal{F}_{y}\left(\left\{\Delta(x-y) \phi_{0}^{p-1}(y)\right\}_{s(y)}\right)=\varphi_{1}(x)
$$

Para obter a última igualdade apenas comparamos com a equação (3.30). Esse resultado mostra que até a ordem $g$, a matriz $S$ conecta os campos $\phi_{i n}$ e $\phi_{\text {em }}$ corretamente, de acordo com (3.29).

Prosseguindo nossa análise, consideremos $n=2$. Segue que

$$
\begin{aligned}
\Phi_{(2)}(x) & =i^{2}\left(\phi_{0}(x) A_{2}-A_{1}^{\dagger} \phi_{0}(x) A_{1}+A_{2}^{\dagger} \phi_{0}(x)\right) \\
& =i^{2} \int d^{D} y_{1} d^{D} y_{2} \mathcal{F}_{12}\left(\theta_{12} \phi_{0}(x) \mathcal{V}\left(\phi_{0}\left(y_{1}\right)\right) \mathcal{V}\left(\phi_{0}\left(y_{2}\right)\right)\right. \\
& \left.-\mathcal{V}\left(\phi_{0}\left(y_{1}\right)\right) \phi_{0}(x) \mathcal{V}\left(\phi_{0}\left(y_{2}\right)\right)+\theta_{12} \mathcal{V}\left(\phi_{0}\left(y_{2}\right)\right) \mathcal{V}\left(\phi_{0}\left(y_{1}\right)\right) \phi_{0}(x)\right)
\end{aligned}
$$

lembrando que a operação $\dagger$, em $A_{2}^{\dagger}$, é aplicada apenas aos campos. Notemos que essa expressão pode ser escrita como

$$
\Phi_{(2)}(x)=i^{2} \int d^{D} y_{1} d^{D} y_{2} \mathcal{F}_{12}\left(\theta_{12}\left[\left[\phi_{0}(x), \mathcal{V}\left(\phi_{0}\left(y_{1}\right)\right)\right], \mathcal{V}\left(\phi_{0}\left(y_{2}\right)\right)\right]\right)
$$

Para ver isso, vamos calcular os comutadores acima:

$$
\begin{aligned}
& \theta_{12}\left[\left[\phi_{0}(x), \mathcal{V}\left(\phi_{0}\left(y_{1}\right)\right)\right], \mathcal{V}\left(\phi_{0}\left(y_{2}\right)\right)\right]=\theta_{12} \phi_{0}(x) \mathcal{V}\left(\phi_{0}\left(y_{1}\right)\right) \mathcal{V}\left(\phi_{0}\left(y_{2}\right)\right) \\
- & \theta_{12} \mathcal{V}\left(\phi_{0}\left(y_{2}\right)\right) \phi_{0}(x) \mathcal{V}\left(\phi_{0}\left(y_{1}\right)\right)-\theta_{12} \mathcal{V}\left(\phi_{0}\left(y_{1}\right)\right) \phi_{0}(x) \mathcal{V}\left(\phi_{0}\left(y_{2}\right)\right) \\
+ & \theta_{12} \mathcal{V}\left(\phi_{0}\left(y_{2}\right)\right) \mathcal{V}\left(\phi_{0}\left(y_{1}\right)\right) \phi_{0}(x)
\end{aligned}
$$

Como $y_{1}$ e $y_{2}$ estão integrados, fazemos a mudança de variável $y_{1} \leftrightarrow y_{2}$ no segundo termo do lado direito, tal que 


$$
\begin{aligned}
& \theta_{12}\left[\left[\phi_{0}(x), \mathcal{V}\left(\phi_{0}\left(y_{1}\right)\right)\right], \mathcal{V}\left(\phi_{0}\left(y_{2}\right)\right)\right] \\
= & \theta_{12} \phi_{0}(x) \mathcal{V}\left(\phi_{0}\left(y_{1}\right)\right) \mathcal{V}\left(\phi_{0}\left(y_{2}\right)\right)-\left(\theta_{21}+\theta_{12}\right) \mathcal{V}\left(\phi_{0}\left(y_{1}\right)\right) \phi_{0}(x) \mathcal{V}\left(\phi_{0}\left(y_{2}\right)\right) \\
+ & \theta_{12} \mathcal{V}\left(\phi_{0}\left(y_{2}\right)\right) \mathcal{V}\left(\phi_{0}\left(y_{1}\right)\right) \phi_{0}(x) \\
= & \theta_{12} \phi_{0}(x) \mathcal{V}\left(\phi_{0}\left(y_{1}\right)\right) \mathcal{V}\left(\phi_{0}\left(y_{2}\right)\right)-\mathcal{V}\left(\phi_{0}\left(y_{1}\right)\right) \phi_{0}(x) \mathcal{V}\left(\phi_{0}\left(y_{2}\right)\right) \\
+ & \theta_{12} \mathcal{V}\left(\phi_{0}\left(y_{2}\right)\right) \mathcal{V}\left(\phi_{0}\left(y_{1}\right)\right) \phi_{0}(x)
\end{aligned}
$$

justificando (3.49). Na última igualdade usamos a indentidade $\theta_{21}+\theta_{12}=1$. O próximo passo é escrever os comutadores em (3.49) de uma forma conveniente. Assim,

$$
\begin{aligned}
{\left[\left[\phi_{0}(x), \phi_{0}^{p}\left(y_{1}\right)\right], \phi_{0}^{p}\left(y_{2}\right)\right] } & =i\left[\left\{\Delta\left(x-y_{1}\right) \phi_{0}^{p-1}\left(y_{1}\right)\right\}_{s\left(y_{1}\right)}, \phi_{0}^{p}\left(y_{2}\right)\right] \\
& =i\left\{\Delta\left(x-y_{1}\right) \phi_{0}^{p-2}\left(y_{1}\right)\left[\phi_{0}\left(y_{1}\right), \phi_{0}^{p}\left(y_{2}\right)\right]\right\}_{s\left(y_{1}\right)} \\
& =-\left\{\Delta\left(x-y_{1}\right) \phi_{0}^{p-2}\left(y_{1}\right)\left\{\Delta\left(y_{1}-y_{2}\right) \phi_{0}^{p-1}\left(y_{2}\right)\right\}_{s\left(y_{2}\right)}\right\}_{s\left(y_{1}\right)} .
\end{aligned}
$$

Na primeira e na última igualdade empregamos o resultado (3.46); na passagem intermediária levamos em conta a simetrização em relação à $y_{1}$. Agora, vamos considerar a presença da função $\theta\left(y_{1}^{0}-y_{2}^{0}\right)$ multiplicando o comutador acima. Nesse ponto entra a realização minimal da função $\theta$, ou seja, devemos escolher uma posição específica para incorporá-la. Explicitamente,

$$
\begin{aligned}
& \theta\left(y_{1}^{0}-y_{2}^{0}\right)\left[\left[\phi_{0}(x), \phi_{0}^{p}\left(y_{1}\right)\right], \phi_{0}^{p}\left(y_{2}\right)\right] \\
= & -\left\{\Delta\left(x-y_{1}\right) \phi_{0}^{p-2}\left(y_{1}\right)\left\{\theta\left(y_{1}^{0}-y_{2}^{0}\right) \Delta\left(y_{1}-y_{2}\right) \phi_{0}^{p-1}\left(y_{2}\right)\right\}_{s\left(y_{2}\right)}\right\}_{s\left(y_{1}\right)} \\
= & \left\{\Delta\left(x-y_{1}\right) \phi_{0}^{p-2}\left(y_{1}\right)\left\{\Delta_{r e t}\left(y_{1}-y_{2}\right) \phi_{0}^{p-1}\left(y_{2}\right)\right\}_{s\left(y_{2}\right)}\right\}_{s\left(y_{1}\right)},
\end{aligned}
$$

em que usamos (3.16) para identificar $\Delta_{r e t}$. Com isso, (3.49) torna-se,

$$
\begin{aligned}
& \Phi_{(2)}(x) \\
= & \frac{g}{p !} \int d^{D} y_{1} \mathcal{F}_{1}\left[\left\{\Delta\left(x-y_{1}\right) \phi_{0}^{p-2}\left(y_{1}\right)\left(-\frac{g}{p !} \int d^{D} y_{2} \mathcal{F}_{2}\left\{\Delta_{r e t}\left(y_{1}-y_{2}\right) \phi_{0}^{p-1}\left(y_{2}\right)\right\}_{s\left(y_{2}\right)}\right)\right\}_{s\left(y_{1}\right)}\right] \\
= & \frac{g}{p !} \int d^{D} y_{1} \mathcal{F}_{1}\left[\left\{\Delta\left(x-y_{1}\right) \phi_{0}^{p-2}\left(y_{1}\right) \phi_{1}\left(y_{1}\right)\right\}_{s\left(y_{1}\right)}\right] \\
= & \varphi_{2}(x) .
\end{aligned}
$$

Nessa expressão, usamos (3.25) e (3.30) para identificar $\phi_{1}$ e $\varphi_{2}$, respectivamente. Sendo assim, vemos que nessa ordem $\left(g^{2}\right)$ a matriz $S$ também fornece a conexão correta entre os campos $\phi_{i n}$ e $\phi_{e m}$. 
Para $n=3$, temos

$$
\begin{aligned}
\Phi_{(3)}(x) & =i^{3}\left(\phi_{0}(x) A_{3}-A_{1}^{\dagger} \phi_{0}(x) A_{3}+A_{2}^{\dagger} \phi_{0}(x) A_{1}-A_{3}^{\dagger} \phi_{0}(x)\right) \\
& =i^{3} \int d^{D} y_{1} d^{D} y_{2} d^{D} y_{3} \mathcal{F}_{123}\left(\theta_{123} \phi_{0}(x) \mathcal{V}\left(\phi_{0}\left(y_{1}\right)\right) \mathcal{V}\left(\phi_{0}\left(y_{2}\right)\right) \mathcal{V}\left(\phi_{0}\left(y_{3}\right)\right)\right. \\
& -\theta_{23} \mathcal{V}\left(\phi_{0}\left(y_{1}\right)\right) \phi_{0}(x) \mathcal{V}\left(\phi_{0}\left(y_{2}\right)\right) \mathcal{V}\left(\phi_{0}\left(y_{3}\right)\right) \\
& +\theta_{12} \mathcal{V}\left(\phi_{0}\left(y_{2}\right)\right) \mathcal{V}\left(\phi_{0}\left(y_{1}\right)\right) \phi_{0}(x) \mathcal{V}\left(\phi_{0}\left(y_{3}\right)\right) \\
& \left.-\theta_{123} \mathcal{V}\left(\phi_{0}\left(y_{3}\right)\right) \mathcal{V}\left(\phi_{0}\left(y_{2}\right)\right) \mathcal{V}\left(\phi_{0}\left(y_{1}\right)\right) \phi_{0}(x)\right)
\end{aligned}
$$

O procedimento aqui é semelhante ao caso anterior. Primeiramente, a equação acima pode ser escrita como

$$
\Phi_{(3)}(x)=i^{3} \int d^{D} y_{1} d^{D} y_{2} d^{D} y_{3} \mathcal{F}_{123}\left(\theta_{123}\left[\left[\left[\phi_{0}(x), \mathcal{V}\left(\phi_{0}\left(y_{1}\right)\right)\right], \mathcal{V}\left(\phi_{0}\left(y_{2}\right)\right)\right], \mathcal{V}\left(\phi_{0}\left(y_{3}\right)\right)\right]\right)
$$

Para ver isso, escrevemos os comutadores de forma aberta,

$$
\begin{aligned}
& \theta_{123}\left[\left[\left[\phi_{0}(x), \mathcal{V}\left(\phi_{0}\left(y_{1}\right)\right)\right], \mathcal{V}\left(\phi_{0}\left(y_{2}\right)\right)\right], \mathcal{V}\left(\phi_{0}\left(y_{3}\right)\right)\right] \\
= & \theta_{123} \phi_{0}(x) \mathcal{V}\left(\phi_{0}\left(y_{1}\right)\right) \mathcal{V}\left(\phi_{0}\left(y_{2}\right)\right) \mathcal{V}\left(\phi_{0}\left(y_{3}\right)\right)-\theta_{123} \mathcal{V}\left(\phi_{0}\left(y_{3}\right)\right) \phi_{0}(x) \mathcal{V}\left(\phi_{0}\left(y_{1}\right)\right) \mathcal{V}\left(\phi_{0}\left(y_{2}\right)\right) \\
- & \theta_{123} \mathcal{V}\left(\phi_{0}\left(y_{2}\right)\right) \phi_{0}(x) \mathcal{V}\left(\phi_{0}\left(y_{1}\right)\right) \mathcal{V}\left(\phi_{0}\left(y_{3}\right)\right)+\theta_{123} \mathcal{V}\left(\phi_{0}\left(y_{3}\right)\right) \mathcal{V}\left(\phi_{0}\left(y_{2}\right)\right) \phi_{0}(x) \mathcal{V}\left(\phi_{0}\left(y_{1}\right)\right) \\
- & \theta_{123} \mathcal{V}\left(\phi_{0}\left(y_{1}\right)\right) \phi_{0}(x) \mathcal{V}\left(\phi_{0}\left(y_{2}\right)\right) \mathcal{V}\left(\phi_{0}\left(y_{3}\right)\right)+\theta_{123} \mathcal{V}\left(\phi_{0}\left(y_{3}\right)\right) \mathcal{V}\left(\phi_{0}\left(y_{1}\right)\right) \phi_{0}(x) \mathcal{V}\left(\phi_{0}\left(y_{2}\right)\right) \\
+ & \theta_{123} \mathcal{V}\left(\phi_{0}\left(y_{2}\right)\right) \mathcal{V}\left(\phi_{0}\left(y_{1}\right)\right) \phi_{0}(x) \mathcal{V}\left(\phi_{0}\left(y_{3}\right)\right)-\theta_{123} \mathcal{V}\left(\phi_{0}\left(y_{3}\right)\right) \mathcal{V}\left(\phi_{0}\left(y_{2}\right)\right) \mathcal{V}\left(\phi_{0}\left(y_{1}\right)\right) \phi_{0}(x)
\end{aligned}
$$

O primeiro e o último termo do lado direiro já estão na forma em que aparecem em (3.55). Analisemos o segundo, o terceiro e o quinto termo:

$$
\begin{aligned}
& -\left(\theta_{123} \mathcal{V}\left(\phi_{0}\left(y_{3}\right)\right) \phi_{0}(x) \mathcal{V}\left(\phi_{0}\left(y_{1}\right)\right) \mathcal{V}\left(\phi_{0}\left(y_{2}\right)\right)+\theta_{123} \mathcal{V}\left(\phi_{0}\left(y_{2}\right)\right) \phi_{0}(x) \mathcal{V}\left(\phi_{0}\left(y_{1}\right)\right) \mathcal{V}\left(\phi_{0}\left(y_{3}\right)\right)\right. \\
+ & \left.\theta_{123} \mathcal{V}\left(\phi_{0}\left(y_{1}\right)\right) \phi_{0}(x) \mathcal{V}\left(\phi_{0}\left(y_{2}\right)\right) \mathcal{V}\left(\phi_{0}\left(y_{3}\right)\right)\right)
\end{aligned}
$$

Como $y_{1}, y_{2}$ e $y_{3}$ estão integrados, fazemos mudanças de variáveis de forma a deixar esses três termos iguais a menos das funções $\theta$, isto é,

$$
-\left(\theta_{231}+\theta_{213}+\theta_{123}\right) \mathcal{V}\left(\phi_{0}\left(y_{1}\right)\right) \phi_{0}(x) \mathcal{V}\left(\phi_{0}\left(y_{2}\right)\right) \mathcal{V}\left(\phi_{0}\left(y_{3}\right)\right)
$$


Agora, usamos a seguinte identidade:

$$
\theta_{231}+\theta_{213}=\theta_{21} \theta_{23}
$$

que pode ser provada facilmente desde que $\theta_{231}$ implica $y_{2}^{0}>y_{3}^{0}>y_{1}^{0}$, tal que podemos multiplicá-la por $\theta_{21}$. Da mesma forma, podemos multiplicar $\theta_{213}$ por $\theta_{23}$ sem alterá-la. Então, partindo do lado esquerdo,

$$
\theta_{23} \theta_{31} \theta_{21}+\theta_{21} \theta_{13} \theta_{23}=\theta_{21} \theta_{23}\left(\theta_{31}+\theta_{13}\right)=\theta_{21} \theta_{23} .
$$

Sendo assim, a equação (3.59) fica,

$$
\begin{aligned}
& -\theta_{23}\left(\theta_{21}+\theta_{12}\right) \mathcal{V}\left(\phi_{0}\left(y_{1}\right)\right) \phi_{0}(x) \mathcal{V}\left(\phi_{0}\left(y_{2}\right)\right) \mathcal{V}\left(\phi_{0}\left(y_{3}\right)\right) \\
= & -\theta_{23} \mathcal{V}\left(\phi_{0}\left(y_{1}\right)\right) \phi_{0}(x) \mathcal{V}\left(\phi_{0}\left(y_{2}\right)\right) \mathcal{V}\left(\phi_{0}\left(y_{3}\right)\right)
\end{aligned}
$$

Esse é um dos termos que aparece em (3.55). Fazendo o mesmo procedimento com os termos remanescentes em (3.57), podemos escrevê-los como

$$
\theta_{12} \mathcal{V}\left(\phi_{0}\left(y_{2}\right)\right) \mathcal{V}\left(\phi_{0}\left(y_{1}\right)\right) \phi_{0}(x) \mathcal{V}\left(\phi_{0}\left(y_{3}\right)\right)
$$

que é o último termo que faltava verificar. Assim, justificamos (3.56). A próxima etapa é o cálculo dos comutadores em (3.56). Esse cálculo foi parcialmente feito em (3.52), restando apenas o comutador $\operatorname{com} \phi_{0}^{p}\left(y_{3}\right)$;

$$
\begin{aligned}
& {\left[\left[\left[\phi_{0}(x), \phi_{0}^{p}\left(y_{1}\right)\right], \phi_{0}^{p}\left(y_{2}\right)\right], \phi_{0}^{p}\left(y_{3}\right)\right] } \\
= & i^{2}\left[\left\{\Delta\left(x-y_{1}\right) \phi_{0}^{p-2}\left(y_{1}\right)\left\{\Delta\left(y_{1}-y_{2}\right) \phi_{0}^{p-1}\left(y_{2}\right)\right\}_{s\left(y_{2}\right)}\right\}_{s\left(y_{1}\right)}, \phi_{0}^{p}\left(y_{3}\right)\right] \\
= & i^{2}\left\{\left[\Delta\left(x-y_{1}\right) \phi_{0}^{p-2}\left(y_{1}\right)\left\{\Delta\left(y_{1}-y_{2}\right) \phi_{0}^{p-1}\left(y_{2}\right)\right\}_{s\left(y_{2}\right)}, \phi_{0}^{p}\left(y_{3}\right)\right]\right\}_{s\left(y_{1}\right)} \\
= & i^{2}\left\{\left[\Delta\left(x-y_{1}\right) \phi_{0}^{p-2}\left(y_{1}\right), \phi_{0}^{p}\left(y_{3}\right)\right]\left\{\Delta\left(y_{1}-y_{2}\right) \phi_{0}^{p-1}\left(y_{2}\right)\right\}_{s\left(y_{2}\right)}\right\}_{s\left(y_{1}\right)} \\
+ & i^{2}\left\{\Delta\left(x-y_{1}\right) \phi_{0}^{p-2}\left(y_{1}\right)\left[\left\{\Delta\left(y_{1}-y_{2}\right) \phi_{0}^{p-1}\left(y_{2}\right)\right\}_{s\left(y_{2}\right)}, \phi_{0}^{p}\left(y_{3}\right)\right]\right\}_{s\left(y_{1}\right)} \\
= & 2 i^{3}\left\{\Delta\left(x-y_{1}\right) \phi_{0}^{p-3}\left(y_{1}\right)\left\{\Delta\left(y_{1}-y_{3}\right) \phi_{0}^{p-1}\left(y_{3}\right)\right\}_{s\left(y_{3}\right)}\left\{\Delta\left(y_{1}-y_{2}\right) \phi_{0}^{p-1}\left(y_{2}\right)\right\}_{s\left(y_{2}\right)}\right\}_{s\left(y_{1}\right)} \\
+ & i^{3}\left\{\Delta\left(x-y_{1}\right) \phi_{0}^{p-2}\left(y_{1}\right)\left\{\Delta\left(y_{1}-y_{2}\right) \phi_{0}^{p-2}\left(y_{2}\right)\left\{\Delta\left(y_{2}-y_{3}\right) \phi_{0}^{p-1}\left(y_{3}\right)\right\}_{s\left(y_{3}\right)}\right\}_{s\left(y_{2}\right)}\right\}_{s\left(y_{1}\right)},
\end{aligned}
$$

em que o fator 2 na última linha vem da simetria entre $y_{2}$ e $y_{3}$ na simetrização. Agora, devemos levar em conta as funções $\theta_{12} \theta_{23}$ multiplicando os comutadores. 


$$
\begin{aligned}
& \theta_{123}\left[\left[\left[\phi_{0}(x), \phi_{0}^{p}\left(y_{1}\right)\right], \phi_{0}^{p}\left(y_{2}\right)\right], \phi_{0}^{p}\left(y_{3}\right)\right] \\
= & 2 i^{3} \theta_{123}\left\{\Delta\left(x-y_{1}\right) \phi_{0}^{p-3}\left(y_{1}\right)\left\{\Delta\left(y_{1}-y_{3}\right) \phi_{0}^{p-1}\left(y_{3}\right)\right\}_{s\left(y_{3}\right)}\left\{\Delta\left(y_{1}-y_{2}\right) \phi_{0}^{p-1}\left(y_{2}\right)\right\}_{s\left(y_{2}\right)}\right\}_{s\left(y_{1}\right)} \\
+ & i^{3} \theta_{123}\left\{\Delta\left(x-y_{1}\right) \phi_{0}^{p-2}\left(y_{1}\right)\left\{\Delta\left(y_{1}-y_{2}\right) \phi_{0}^{p-2}\left(y_{2}\right)\left\{\Delta\left(y_{2}-y_{3}\right) \phi_{0}^{p-1}\left(y_{3}\right)\right\}_{s\left(y_{3}\right)}\right\}_{s\left(y_{2}\right)}\right\}_{s\left(y_{1}\right)} \\
= & i^{3}\left(\theta_{123}+\theta_{132}\right)\left\{\Delta\left(x-y_{1}\right) \phi_{0}^{p-3}\left(y_{1}\right)\left\{\Delta\left(y_{1}-y_{3}\right) \phi_{0}^{p-1}\left(y_{3}\right)\right\}_{s\left(y_{3}\right)}\right. \\
\times & \left.\left\{\Delta\left(y_{1}-y_{2}\right) \phi_{0}^{p-1}\left(y_{2}\right)\right\}_{s\left(y_{2}\right)}\right\}_{s\left(y_{1}\right)} \\
+ & i^{3} \theta_{123}\left\{\Delta\left(x-y_{1}\right) \phi_{0}^{p-2}\left(y_{1}\right)\left\{\Delta\left(y_{1}-y_{2}\right) \phi_{0}^{p-2}\left(y_{2}\right)\left\{\Delta\left(y_{2}-y_{3}\right) \phi_{0}^{p-1}\left(y_{3}\right)\right\}_{s\left(y_{3}\right)}\right\}_{s\left(y_{2}\right)}\right\}_{s\left(y_{1}\right)} .
\end{aligned}
$$

Na última igualdade, usando o fato que as variáveis $y_{1}, y_{2}$ e $y_{3}$ estão sendo integradas, fizemos a mudança de variável $y_{2} \leftrightarrow y_{3}$, explorando a simetria entre $y_{2}$ e $y_{3}$. Empregando a identidade (3.60), vamos trocar a soma $\theta_{123}+\theta_{132}$ por $\theta_{12} \theta_{13}$. Essa troca é conveniente pois, dentro da realização minimal, escolhemos incorporar $\theta_{12}$ à função $\Delta\left(y_{1}-y_{2}\right)$ originando a função retardada $\Delta_{r e t}\left(y_{1}-y_{2}\right)$; e $\theta_{13}$ à $\Delta\left(y_{1}-y_{3}\right)$ originando $\Delta_{r e t}\left(y_{1}-y_{3}\right)$. No segundo termo incorporamos as funções $\theta_{12}$ e $\theta_{23}$ com as seguintes escolhas: $\theta_{12} \Delta\left(y_{1}-y_{2}\right)=$ $-\Delta_{r e t}\left(y_{1}-y_{2}\right)$ e $\theta_{23} \Delta\left(y_{2}-y_{3}\right)=-\Delta_{r e t}\left(y_{2}-y_{3}\right)$. Como descrito,

$$
\begin{aligned}
& \theta_{123}\left[\left[\left[\phi_{0}(x), \phi_{0}^{p}\left(y_{1}\right)\right], \phi_{0}^{p}\left(y_{2}\right)\right], \phi_{0}^{p}\left(y_{3}\right)\right] \\
= & i^{3}\left\{\Delta\left(x-y_{1}\right) \phi_{0}^{p-3}\left(y_{1}\right)\left\{\Delta_{r e t}\left(y_{1}-y_{3}\right) \phi_{0}^{p-1}\left(y_{3}\right)\right\}_{s\left(y_{3}\right)}\left\{\Delta_{r e t}\left(y_{1}-y_{2}\right) \phi_{0}^{p-1}\left(y_{2}\right)\right\}_{s\left(y_{2}\right)}\right\}_{s\left(y_{1}\right)} \\
+ & i^{3}\left\{\Delta\left(x-y_{1}\right) \phi_{0}^{p-2}\left(y_{1}\right)\left\{\Delta_{r e t}\left(y_{1}-y_{2}\right) \phi_{0}^{p-2}\left(y_{2}\right)\left\{\Delta_{r e t}\left(y_{2}-y_{3}\right) \phi_{0}^{p-1}\left(y_{3}\right)\right\}_{s\left(y_{3}\right)}\right\}_{s\left(y_{2}\right)}\right\}_{s\left(y_{1}\right)} .
\end{aligned}
$$

Substituindo esse resultado em (3.56), ficamos com 


$$
\begin{aligned}
& \Phi_{(3)}(x) \\
= & -i^{3}\left(\frac{g}{p !}\right)^{3} \int d^{D} y_{1} d^{D} y_{2} d^{D} y_{3} \mathcal{F}_{123}\left(i ^ { 3 } \left\{\Delta\left(x-y_{1}\right) \phi_{0}^{p-3}\left(y_{1}\right)\right.\right. \\
\times & \left.\left\{\Delta_{r e t}\left(y_{1}-y_{3}\right) \phi_{0}^{p-1}\left(y_{3}\right)\right\}_{s\left(y_{3}\right)}\left\{\Delta_{r e t}\left(y_{1}-y_{2}\right) \phi_{0}^{p-1}\left(y_{2}\right)\right\}_{s\left(y_{2}\right)}\right\}_{s\left(y_{1}\right)} \\
+ & \left.i^{3}\left\{\Delta\left(x-y_{1}\right) \phi_{0}^{p-2}\left(y_{1}\right)\left\{\Delta_{r e t}\left(y_{1}-y_{2}\right) \phi_{0}^{p-2}\left(y_{2}\right)\left\{\Delta_{r e t}\left(y_{2}-y_{3}\right) \phi_{0}^{p-1}\left(y_{3}\right)\right\}_{s\left(y_{3}\right)}\right\}_{s\left(y_{2}\right)}\right\}_{s\left(y_{1}\right)}\right) \\
= & \frac{g}{p !} \int d^{D} y_{1} \mathcal{F}_{1}\left\{\Delta\left(x-y_{1}\right) \phi_{0}^{p-3}\left(y_{1}\right) \int d^{D} y_{3} \mathcal{F}_{3}\left\{-\frac{g}{p !} \Delta_{r e t}\left(y_{1}-y_{3}\right) \phi_{0}^{p-1}\left(y_{3}\right)\right\}_{s\left(y_{3}\right)}\right. \\
\times & \int d^{D} y_{2} \mathcal{F}_{2}\left\{-\frac{g}{p !} \Delta_{r e t}\left(y_{1}-y_{2}\right) \phi_{0}^{p-1}\left(y_{2}\right)\right\}_{s\left(y_{2}\right)} \\
+ & \frac{g}{p !} \int d^{D} y_{1} \mathcal{F}_{1}\left\{\Delta ( x - y _ { 1 } ) \phi _ { 0 } ^ { p - 2 } ( y _ { 1 } ) \int d ^ { D } y _ { 2 } \mathcal { F } _ { 2 } \left\{-\frac{g}{p !} \Delta_{r e t}\left(y_{1}-y_{2}\right) \phi_{0}^{p-2}\left(y_{2}\right)\right.\right. \\
\times & \left.\left.\int d^{D} y_{3} \mathcal{F}_{3}\left\{-\frac{g}{p !} \Delta_{r e t}\left(y_{2}-y_{3}\right) \phi_{0}^{p-1}\left(y_{3}\right)\right\}_{s\left(y_{3}\right)}\right\}_{s\left(y_{2}\right)}\right\}_{s\left(y_{1}\right)} \\
= & \frac{g}{p !} \int d^{D} y_{1} \mathcal{F}_{1}\left\{\Delta\left(x-y_{1}\right)\left(\phi_{0}^{p-3}\left(y_{1}\right) \phi_{1}^{2}\left(y_{1}\right)+\phi_{0}^{p-2}\left(y_{1}\right) \phi_{2}\left(y_{1}\right)\right)\right\}_{s\left(y_{1}\right)} \\
= & \varphi_{3}(x),
\end{aligned}
$$

sendo que na terceira igualdade usamos as definições de $\phi_{1}$ e $\phi_{2}$ em (3.25) e (3.26), e na última igualdade a definição de $\varphi_{3}$ dada em (3.30). Com esse resultado concluímos nossa verificação de que a matriz $S$ conecta os campos $\phi_{i n}$ e $\phi_{e m}$ de maneira correta até terceira ordem em $g$. Cálculos em ordens superiores seguem de maneira análoga.

\subsection{Unitariedade}

Prosseguindo na verificação da consistência da matriz $S$, partimos para o estudo da unitariedade. Devemos então verificar a relação

$$
S^{\dagger} S=S S^{\dagger}=1
$$

ordem por ordem em $g$. Tal como na seção anterior conduziremos nossos cálculos até a ordem $g^{3}$.

A condição de unitariedade em ordem $g$, com $S$ dada por (3.36), é

$$
A_{1}=A_{1}^{\dagger}
$$

que é trivialmente satisfeita. 
Em ordem $g^{2}$, temos a condição

$$
A_{2}+A_{2}^{\dagger}=A_{1}^{\dagger} A_{1} .
$$

Partindo do lado esquerdo, segue que

$$
\begin{aligned}
A_{2}+A_{2}^{\dagger} & =\int d^{D} y_{1} d^{D} y_{2} \mathcal{F}_{12}\left[\theta_{12}\left(\mathcal{V}\left(\phi_{0}\left(y_{1}\right)\right) \mathcal{V}\left(\phi_{0}\left(y_{2}\right)\right)+\mathcal{V}\left(\phi_{0}\left(y_{2}\right)\right) \mathcal{V}\left(\phi_{0}\left(y_{1}\right)\right)\right)\right] \\
& =\int d^{D} y_{1} d^{D} y_{2} \mathcal{F}_{12}\left[\left(\theta_{12}+\theta_{21}\right) \mathcal{V}\left(\phi_{0}\left(y_{1}\right)\right) \mathcal{V}\left(\phi_{0}\left(y_{2}\right)\right)\right] \\
& =\int d^{D} y_{1} \mathcal{F}_{1}\left(\mathcal{V}\left(\phi_{0}\left(y_{1}\right)\right)\right) \int d^{D} y_{2} \mathcal{F}_{2}\left(\mathcal{V}\left(\phi_{0}\left(y_{2}\right)\right)\right) \\
& =A_{1}^{\dagger} A_{1} .
\end{aligned}
$$

Fizemos a mudança de variável $y_{1} \leftrightarrow y_{2}$ no segundo termo da primeira igualdade para obter a segunda linha. Esse resultado mostra mostra a condição de unitariedade.

A condição em ordem $g^{3}$ é a seguinte:

$$
A_{3}-A_{3}^{\dagger}=A_{1}^{\dagger} A_{2}-A_{2}^{\dagger} A_{1}
$$

O lado esquerdo é dado por,

$$
\begin{aligned}
A_{3}-A_{3}^{\dagger} & =\int d^{D} y_{1} d^{D} y_{2} d^{D} y_{3} \mathcal{F}_{123}\left[\theta _ { 1 2 3 } \left(\mathcal{V}\left(\phi_{0}\left(y_{1}\right)\right) \mathcal{V}\left(\phi_{0}\left(y_{2}\right)\right) \mathcal{V}\left(\phi_{0}\left(y_{3}\right)\right)\right.\right. \\
& \left.\left.-\mathcal{V}\left(\phi_{0}\left(y_{3}\right)\right) \mathcal{V}\left(\phi_{0}\left(y_{2}\right)\right) \mathcal{V}\left(\phi_{0}\left(y_{1}\right)\right)\right)\right] \\
& =\int d^{D} y_{1} d^{D} y_{2} d^{D} y_{3} \mathcal{F}_{123}\left[\left(\theta_{123}-\theta_{321}\right) \mathcal{V}\left(\phi_{0}\left(y_{1}\right)\right) \mathcal{V}\left(\phi_{0}\left(y_{2}\right)\right) \mathcal{V}\left(\phi_{0}\left(y_{3}\right)\right)\right] .
\end{aligned}
$$

Para obter a última linha fizemos a mudança $y_{1} \leftrightarrow y_{3}$ no segundo termo da linha anterior. Notemos que

$$
\begin{aligned}
\theta_{123}-\theta_{321} & =\theta_{12} \theta_{23}-\left(1-\theta_{23}\right) \theta_{21} \\
& =\theta_{23}-\theta_{21}
\end{aligned}
$$

Substituindo em (3.73), ficamos com 


$$
\begin{aligned}
A_{3}-A_{3}^{\dagger} & =\int d^{D} y_{1} d^{D} y_{2} d^{D} y_{3} \mathcal{F}_{123}\left[\left(\theta_{23}-\theta_{21}\right) \mathcal{V}\left(\phi_{0}\left(y_{1}\right)\right) \mathcal{V}\left(\phi_{0}\left(y_{2}\right)\right) \mathcal{V}\left(\phi_{0}\left(y_{3}\right)\right)\right] \\
& =\int d^{D} y_{1} \mathcal{F}_{1}\left(\mathcal{V}\left(\phi_{0}\left(y_{1}\right)\right)\right) \int d^{D} y_{2} d^{D} y_{3} \mathcal{F}_{23}\left(\theta_{23} \mathcal{V}\left(\phi_{0}\left(y_{2}\right)\right) \mathcal{V}\left(\phi_{0}\left(y_{3}\right)\right)\right) \\
& -\int d^{D} y_{1} d^{D} y_{2} \mathcal{F}_{21}\left(\theta_{21} \mathcal{V}\left(\phi_{0}\left(y_{1}\right)\right) \mathcal{V}\left(\phi_{0}\left(y_{2}\right)\right)\right) \int d^{D} y_{3} \mathcal{F}_{3}\left(\mathcal{V}\left(\phi_{0}\left(y_{3}\right)\right)\right) \\
& =A_{1}^{\dagger} A_{2}-A_{2}^{\dagger} A_{1},
\end{aligned}
$$

verificando assim a condição de unitariedade. A análise de ordens superiores segue de maneira semelhante.

Agora que verificamos a consistência da matriz $S$, podemos empregar o formalismo desenvolvido para efetuar cálculos de unitariedade em modelos específicos. Esse assunto é abordado nos capítulos seguintes. 


\section{Capítulo 4}

\section{Modelo $\phi^{3}$ Não Comutativo}

Neste capítulo, vamos estudar novamente o modelo $\phi^{3}$ de acordo com os desenvolvimentos feitos no capítulo anterior. Esse estudo é constituído essencialmente da verificação explícita da condição de unitariedade em segunda ordem na constante de acoplamento, (diagrama próprio de um laço).

\subsection{Unitariedade até um laço}

A lagrangiana de interação é dada por

$$
L_{I}(t)=-\frac{g}{3 !} \int d^{D-1} x \phi_{*}^{3}(x, t) .
$$

Nessa nova investigação da unitariedade no modelo $\phi^{3}$, consideraremos um processo em que uma partícula incide com momento $p_{1}$ e uma partícula emerge com momento $p_{2}$ e que contribui para o diagrama mostrado na figura (4.1). Queremos então verificar a condição

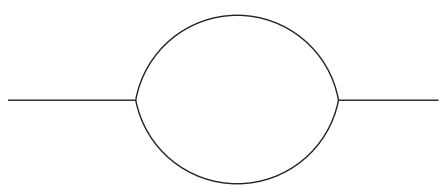

Figura 4.1: Diagrama de um laço.

de unitariedade da matriz $S$ em segunda ordem em $g$, que é justamente a relação (3.42). Dito de outra forma, queremos verificar a seguinte igualdade entre amplitudes:

$$
\left\langle p_{1}\left|A_{2}+A_{2}^{\dagger}\right| p_{2}\right\rangle=\left\langle p_{1}\left|A_{1}^{\dagger} A_{1}\right| p_{2}\right\rangle,
$$


considerando apenas termos que contribuem para o diagrama (4.1).

Nos concentremos inicialmente em $\left\langle p_{1}\left|A_{2}\right| p_{2}\right\rangle$. De acordo com (3.36), temos,

$$
\begin{aligned}
\left\langle p_{1}\left|A_{2}\right| p_{2}\right\rangle & =\left\langle p_{1}\left|\int d^{D} x d^{D} y \mathcal{F}_{x y}\left[\theta_{x y} \mathcal{V}\left(\phi_{0}(x)\right) \mathcal{V}\left(\phi_{0}(y)\right)\right]\right| p_{2}\right\rangle \\
& =\left(\frac{g}{3 !}\right)^{2} \int d^{D} x d^{D} y\left\langle p_{1}\left|\mathcal{F}_{x y}\left[\theta\left(x^{0}-y^{0}\right) \phi_{0}^{3}(x) \phi_{0}^{3}(y)\right]\right| p_{2}\right\rangle
\end{aligned}
$$

Usando a representação integral para a função $\theta$,

$$
\theta(t)=-\int_{-\infty}^{\infty} \frac{d \omega}{2 \pi i} \frac{e^{-i \omega t}}{\omega+i \epsilon},
$$

podemos escrever, lembrando da prescrição (3.39),

$$
\begin{aligned}
\left\langle p_{1}\left|A_{2}\right| p_{2}\right\rangle & =-\left(\frac{g}{3 !}\right)^{2} \int d^{D} x d^{D} y \int \frac{d \omega}{2 \pi i} \frac{1}{\omega+i \epsilon}\left\langle p_{1}\left|\mathcal{F}_{x y}\left[e^{-i \omega\left(x^{0}-y^{0}\right)} \phi_{0}^{3}(x) \phi_{0}^{3}(y)\right]\right| p_{2}\right\rangle \\
& =-\left(\frac{g}{3 !}\right)^{2} \int d^{D} x d^{D} y \int \frac{d \omega}{2 \pi i} \frac{1}{\omega+i \epsilon} \\
& \times\left\langle p_{1}\left|\mathcal{F}_{x y}\left[e^{-i \omega\left(x_{i}^{0}-y_{j}^{0}\right)} \phi_{0}\left(x_{1}\right) \phi_{0}\left(x_{2}\right) \phi_{0}\left(x_{3}\right) \phi_{0}\left(y_{1}\right) \phi_{0}\left(y_{2}\right) \phi_{0}\left(y_{3}\right)\right]\right| p_{2}\right\rangle .
\end{aligned}
$$

Na última linha estamos a ponto de implementar a realização minimal. Dentro desse esquema, os campos escolhidos para incorporar a função $\theta$ deverão estar contraídos dando origem a alguma das funções relativas ao campo escalar $\left(\Delta, \Delta^{+}, \cdots\right)$. Essas contrações serão feitas posteriormente quando aplicamos o teorema de Wick. Fazemos então a seguinte escolha:

$$
\begin{aligned}
\left\langle p_{1}\left|A_{2}\right| p_{2}\right\rangle & =-\left(\frac{g}{3 !}\right)^{2} \int d^{D} x d^{D} y \int \frac{d \omega}{2 \pi i} \frac{1}{\omega+i \epsilon} \\
& \times\left\langle p_{1}\left|\mathcal{F}_{x y}\left[\phi_{0}\left(x_{1}\right) \phi_{0}\left(x_{2}\right)\left(e^{-i \omega x_{3}^{0}} \phi_{0}\left(x_{3}\right)\right) \phi_{0}\left(y_{1}\right) \phi_{0}\left(y_{2}\right)\left(e^{i \omega y_{3}} \phi_{0}\left(y_{3}\right)\right)\right]\right| p_{2}\right\rangle \\
& =-\left(\frac{g}{3 !}\right)^{2} \int d^{D} x d^{D} y \int \frac{d \omega}{2 \pi i} \frac{1}{\omega+i \epsilon} \\
& \times\left\langle p_{1}\left|\left(\phi_{0}(x) * \phi_{0}(x) * \varphi_{0}(x)\right)\left(\phi_{0}(y) * \phi_{0}(y) * \psi_{0}(y)\right)\right| p_{2}\right\rangle
\end{aligned}
$$

com $\varphi_{0}(x) \equiv e^{-i \omega x} \phi_{0}(x)$ e $\psi_{0}(y) \equiv e^{i \omega y} \phi_{0}(y)$, tal que definimos o quadrivetor $\omega^{\mu} \equiv$ $(\omega, 0,0,0)$. Agora, usamos (1.45) para escrever a expressão acima como, 


$$
\begin{aligned}
\left\langle p_{1}\left|A_{2}\right| p_{2}\right\rangle & =-\left(\frac{g}{3 !}\right)^{2} \int \frac{d \omega}{2 \pi i} \frac{1}{\omega+i \epsilon} \prod_{i=1}^{3} \int \frac{d^{D} k_{i}}{(2 \pi)^{D}} \prod_{j=1}^{3} \int \frac{d^{D} q_{j}}{(2 \pi)^{D}} \\
& \times(2 \pi)^{D} \delta^{D}\left(k_{1}+k_{2}+k_{3}\right) \cos \left(\frac{k_{1} \wedge k_{2}}{2}\right)(2 \pi)^{D} \delta^{D}\left(q_{1}+q_{2}+q_{3}\right) \cos \left(\frac{q_{1} \wedge q_{2}}{2}\right) \\
& \times\left\langle p_{1}\left|\widetilde{\phi}_{0}\left(k_{1}\right) \widetilde{\phi}_{0}\left(k_{2}\right) \widetilde{\varphi}_{0}\left(k_{3}\right) \widetilde{\phi}_{0}\left(q_{1}\right) \widetilde{\phi}_{0}\left(q_{2}\right) \widetilde{\psi}_{0}\left(q_{3}\right)\right| p_{2}\right\rangle .
\end{aligned}
$$

É importante observar que,

$$
\begin{aligned}
\widetilde{\varphi}_{0}\left(k_{3}\right) & =\int d^{D} x_{3} e^{i k_{3} x_{3}}\left(e^{-i \omega x_{3}} \phi_{0}\left(x_{3}\right)\right) \\
& =\widetilde{\phi}_{0}\left(k_{3}-\omega\right) .
\end{aligned}
$$

Da mesma forma,

$$
\widetilde{\psi}_{0}\left(q_{3}\right)=\widetilde{\phi}_{0}\left(q_{3}+\omega\right)
$$

Assim, resta-nos calcular o elemento de matriz:

$$
\left\langle p_{1}\left|\widetilde{\phi_{0}}\left(k_{1}\right) \widetilde{\phi_{0}}\left(k_{2}\right) \widetilde{\phi_{0}}\left(k_{3}-\omega\right) \widetilde{\phi_{0}}\left(q_{1}\right) \widetilde{\phi_{0}}\left(q_{2}\right) \widetilde{\phi_{0}}\left(q_{3}+\omega\right)\right| p_{2}\right\rangle
$$

Para esse cálculo algumas explicações são necessárias: Primeiramente vamos usar o teorema de Wick para um produto ordinário entre campos bosônicos (sem ordenamento temporal),

$$
\begin{aligned}
& \phi\left(z_{1}\right) \phi\left(z_{2}\right) \phi\left(z_{3}\right) \cdots \phi\left(z_{N}\right)=: \phi\left(z_{1}\right) \phi\left(z_{2}\right) \phi\left(z_{3}\right) \cdots \phi\left(z_{N}\right): \\
+ & : \phi^{c_{1}}\left(z_{1}\right) \phi^{c_{1}}\left(z_{2}\right) \phi\left(z_{3}\right) \cdots \phi\left(z_{N}\right):+: \phi^{c_{1}}\left(z_{1}\right) \phi\left(z_{2}\right) \phi^{c_{1}}\left(z_{3}\right) \cdots \phi\left(z_{N}\right):+\cdots \\
+ & : \phi^{c_{1}}\left(z_{1}\right) \phi^{c_{1}}\left(z_{2}\right) \phi^{c_{2}}\left(z_{3}\right) \phi^{c_{2}}\left(z_{4}\right) \cdots \phi\left(z_{N}\right):+\cdots \\
+ & : \phi^{c_{1}}\left(z_{1}\right) \phi^{c_{1}}\left(z_{2}\right) \phi^{c_{2}}\left(z_{3}\right) \phi^{c_{2}}\left(z_{4}\right) \cdots \phi^{c_{n}}\left(z_{N-1}\right) \phi^{c_{n}}\left(z_{N}\right):
\end{aligned}
$$

em que os superescritos $c_{i}$ servem para indicar que dois campos com um mesmo índice superior estão contraídos. No primeiro termo do lado direito temos o produto de todos os campos com ordenamento de Wick. A segunda linha representa todas as maneiras possíveis de contrair dois campos, ou seja, apenas com uma contração. A segunda linha representa todas as possibilidades de realizar duas contrações. Esse processo segue até que todos os campos estejam contraídos de todas as maneiras possíveis. Esse é o significado de (4.11). Além disso, empregamos a notação:

$: \phi\left(z_{1}\right) \cdots \phi^{c_{1}}\left(z_{i}\right) \cdots \phi^{c_{1}}\left(z_{j}\right) \cdots \phi\left(z_{N}\right): \equiv \phi^{c_{1}}\left(z_{i}\right) \phi^{c_{1}}\left(z_{j}\right): \phi\left(z_{1}\right) \cdots \hat{\phi}\left(z_{i}\right) \cdots \hat{\phi}\left(z_{j}\right) \cdots \phi\left(z_{N}\right):$. 
$\hat{\phi}$ indica a ausência desse campo no produto. A contração de dois campos é dada por,

$$
\phi_{0}^{c_{1}}\left(z_{1}\right) \phi_{0}^{c_{1}}\left(z_{2}\right) \equiv\left\langle 0\left|\phi_{0}\left(z_{1}\right) \phi_{0}\left(z_{2}\right)\right| 0\right\rangle=\Delta^{+}\left(z_{1}-z_{2}\right) .
$$

$\Delta^{+}$foi definido na equação (1.7). Notemos, entretanto, que as contrações acima estão sendo feitas no espaço coordenado ao passo que o elemento de matriz que temos que temos que avaliar está no espaço dos momentos. Uma contração no espaço dos momentos é obtida como segue;

$$
\begin{aligned}
\widetilde{\phi}_{0}^{c_{1}}(k) \widetilde{\phi}_{0}^{c_{1}}(q) & =\int d^{D} x d^{D} y e^{i k x} e^{i q y} \phi_{0}^{c_{1}}(x) \phi_{0}^{c_{1}}(y) \\
& =\int d^{D} x d^{D} y e^{i k x} e^{i q y} \Delta^{+}(x-y) \\
& =\int \frac{d^{D} l}{(2 \pi)^{D}} \widetilde{\Delta}^{+}(l)(2 \pi)^{D} \delta^{D}(k-l)(2 \pi)^{D} \delta^{D}(q+l) \\
& =(2 \pi)^{D} \delta^{D}(k+q) \widetilde{\Delta}^{+}(k) .
\end{aligned}
$$

Com os resultados acima, temos condições de avaliar e elemento de matriz (4.10). Inicialmente, observamos que apenas os termos com duas contrações no teorema de Wick contribuem para o diagrama da figura (4.1); além disso, em cada contração um dos campos deve ter argumento pertencente ao conjunto $\left\{k_{1}, k_{2}, k_{3}-\omega\right\}$ e o outro pertencente a $\left\{q_{1}, q_{2}, q_{3}+\omega\right\}$. Lembremos que dentro da realização minimal, os campos em que a função $\theta$ foi incorporada deverão estar contraídos entre si. Então, para a escolha particular que fizemos na realização minimal, teremos os seguintes termos:

$$
\begin{aligned}
& \left\langle p_{1}\left|\widetilde{\phi}_{0}\left(k_{1}\right) \widetilde{\phi}_{0}\left(k_{2}\right) \widetilde{\phi}_{0}\left(k_{3}-\omega\right) \widetilde{\phi}_{0}\left(q_{1}\right) \widetilde{\phi}_{0}\left(q_{2}\right) \widetilde{\phi}_{0}\left(q_{3}+\omega\right)\right| p_{2}\right\rangle \\
= & \widetilde{\phi}_{0}^{c_{1}}\left(k_{1}\right) \widetilde{\phi}_{0}^{c_{1}}\left(q_{1}\right) \widetilde{\phi}_{0}^{c_{2}}\left(k_{3}-\omega\right) \widetilde{\phi}_{0}^{c_{2}}\left(q_{3}+\omega\right)\left\langle p_{1}\left|: \widetilde{\phi}_{0}\left(k_{2}\right) \widetilde{\phi}_{0}\left(q_{2}\right):\right| p_{2}\right\rangle \\
+ & \widetilde{\phi}_{0}^{c_{1}}\left(k_{1}\right) \widetilde{\phi}_{0}^{c_{1}}\left(q_{2}\right) \widetilde{\phi}_{0}^{c_{2}}\left(k_{3}-\omega\right) \widetilde{\phi}_{0}^{c_{2}}\left(q_{3}+\omega\right)\left\langle p_{1}\left|: \widetilde{\phi}_{0}\left(k_{2}\right) \widetilde{\phi}_{0}\left(q_{1}\right):\right| p_{2}\right\rangle \\
+ & \widetilde{\phi}_{0}^{c_{1}}\left(k_{2}\right) \widetilde{\phi}_{0}^{c_{1}}\left(q_{1}\right) \widetilde{\phi}_{0}^{c_{2}}\left(k_{3}-\omega\right) \widetilde{\phi}_{0}^{c_{2}}\left(q_{3}+\omega\right)\left\langle p_{1}\left|: \widetilde{\phi}_{0}\left(k_{1}\right) \widetilde{\phi}_{0}\left(q_{2}\right):\right| p_{2}\right\rangle \\
+ & \widetilde{\phi}_{0}^{c_{1}}\left(k_{2}\right) \widetilde{\phi}_{0}^{c_{1}}\left(q_{2}\right) \widetilde{\phi}_{0}^{c_{2}}\left(k_{3}-\omega\right) \widetilde{\phi}_{0}^{c_{2}}\left(q_{3}+\omega\right)\left\langle p_{1}\left|: \widetilde{\phi}_{0}\left(k_{1}\right) \widetilde{\phi}_{0}\left(q_{1}\right):\right| p_{2}\right\rangle .
\end{aligned}
$$

Porém, poderíamos ter escolhido incorporar a função $\theta$, em (4.6), de maneira diferente, mais precisamente, de $3 \times 3=9$ maneiras diferentes. Cada uma dessas escolhas dá origem a quatro termos, como visto na expressão acima. Desse modo, o número total é $36=(3 !)^{2}$ termos. Por outro lado, como todos os argumentos dos campos estão integrados, é possível verificar que todos esses termos dão a mesma contribuição para (4.7). Para ilustrar esse fato, examinemos uma outra escolha para a realização minimal, de modo que o elemento de matriz é 


$$
\begin{aligned}
& \left\langle p_{1}\left|\widetilde{\phi}_{0}\left(k_{1}-\omega\right) \widetilde{\phi}_{0}\left(k_{2}\right) \widetilde{\phi}_{0}\left(k_{3}\right) \widetilde{\phi}_{0}\left(q_{1}\right) \widetilde{\phi}_{0}\left(q_{2}+\omega\right) \widetilde{\phi}_{0}\left(q_{3}\right)\right| p_{2}\right\rangle \\
= & \widetilde{\phi}_{0}^{c_{1}}\left(k_{2}\right) \widetilde{\phi}_{0}^{c_{1}}\left(q_{1}\right) \widetilde{\phi}_{0}^{c_{2}}\left(k_{1}-\omega\right) \widetilde{\phi}_{0}^{c_{2}}\left(q_{2}+\omega\right)\left\langle p_{1}\left|: \widetilde{\phi}_{0}\left(k_{3}\right) \widetilde{\phi}_{0}\left(q_{3}\right):\right| p_{2}\right\rangle \\
+ & \widetilde{\phi}_{0}^{c_{1}}\left(k_{2}\right) \widetilde{\phi}_{0}^{c_{1}}\left(q_{3}\right) \widetilde{\phi}_{0}^{c_{2}}\left(k_{1}-\omega\right) \widetilde{\phi}_{0}^{c_{2}}\left(q_{2}+\omega\right)\left\langle p_{1}\left|: \widetilde{\phi}_{0}\left(k_{3}\right) \widetilde{\phi}_{0}\left(q_{1}\right):\right| p_{2}\right\rangle \\
+ & \widetilde{\phi}_{0}^{c_{1}}\left(k_{3}\right) \widetilde{\phi}_{0}^{c_{1}}\left(q_{1}\right) \widetilde{\phi}_{0}^{c_{2}}\left(k_{1}-\omega\right) \widetilde{\phi}_{0}^{c_{2}}\left(q_{2}+\omega\right)\left\langle p_{1}\left|: \widetilde{\phi}_{0}\left(k_{2}\right) \widetilde{\phi}_{0}\left(q_{3}\right):\right| p_{2}\right\rangle \\
+ & \widetilde{\phi}_{0}^{c_{1}}\left(k_{3}\right) \widetilde{\phi}_{0}^{c_{1}}\left(q_{3}\right) \widetilde{\phi}_{0}^{c_{2}}\left(k_{1}-\omega\right) \widetilde{\phi}_{0}^{c_{2}}\left(q_{2}+\omega\right)\left\langle p_{1}\left|: \widetilde{\phi}_{0}\left(k_{2}\right) \widetilde{\phi}_{0}\left(q_{1}\right):\right| p_{2}\right\rangle .
\end{aligned}
$$

É suficiente considerar apenas um desses quatro termos, por exemplo, o segundo termo do lado direito. Incluindo também os fatores de coseno multiplicando, temos

$$
\cos \left(\frac{k_{1} \wedge k_{2}}{2}\right) \cos \left(\frac{q_{1} \wedge q_{2}}{2}\right) \widetilde{\phi}_{0}^{c_{1}}\left(k_{2}\right) \widetilde{\phi}_{0}^{c_{1}}\left(q_{3}\right) \widetilde{\phi}_{0}^{c_{2}}\left(k_{1}-\omega\right) \widetilde{\phi}_{0}^{c_{2}}\left(q_{2}+\omega\right)\left\langle p_{1}\left|: \widetilde{\phi}_{0}\left(k_{3}\right) \widetilde{\phi}_{0}\left(q_{1}\right):\right| p_{2}\right\rangle
$$

As primeiras mudanças de variáveis são $k_{1} \leftrightarrow k_{2}$ e $q_{1} \leftrightarrow q_{3}$. As funções deltas não são alteradas por tais mudanças. Sendo assim, (4.17) torna-se

$$
\begin{aligned}
& \cos \left(\frac{k_{2} \wedge k_{1}}{2}\right) \cos \left(\frac{q_{3} \wedge q_{2}}{2}\right) \widetilde{\phi}_{0}^{c_{1}}\left(k_{1}\right) \widetilde{\phi}_{0}^{c_{1}}\left(q_{1}\right) \widetilde{\phi}_{0}^{c_{2}}\left(k_{2}-\omega\right) \widetilde{\phi}_{0}^{c_{2}}\left(q_{2}+\omega\right) \\
\times & \left\langle p_{1}\left|: \widetilde{\phi}_{0}\left(k_{3}\right) \widetilde{\phi}_{0}\left(q_{3}\right):\right| p_{2}\right\rangle \\
= & \cos \left(\frac{k_{1} \wedge k_{2}}{2}\right) \cos \left(\frac{q_{1} \wedge q_{2}}{2}\right) \widetilde{\phi}_{0}^{c_{1}}\left(k_{1}\right) \widetilde{\phi}_{0}^{c_{1}}\left(q_{1}\right) \widetilde{\phi}_{0}^{c_{2}}\left(k_{2}-\omega\right) \widetilde{\phi}_{0}^{c_{2}}\left(q_{2}+\omega\right) \\
\times & \left\langle p_{1}\left|: \widetilde{\phi}_{0}\left(k_{3}\right) \widetilde{\phi}_{0}\left(q_{3}\right):\right| p_{2}\right\rangle .
\end{aligned}
$$

O argumento do coseno foi alterado usando uma função delta. As próximas mudanças são $k_{2} \leftrightarrow k_{3}$ e $q_{2} \leftrightarrow q_{3}$, tal que

$$
\begin{aligned}
& \cos \left(\frac{k_{1} \wedge k_{3}}{2}\right) \cos \left(\frac{q_{1} \wedge q_{3}}{2}\right) \widetilde{\phi}_{0}^{c_{1}}\left(k_{1}\right) \widetilde{\phi}_{0}^{c_{1}}\left(q_{1}\right) \widetilde{\phi}_{0}^{c_{2}}\left(k_{3}-\omega\right) \widetilde{\phi}_{0}^{c_{2}}\left(q_{3}+\omega\right) \\
\times & \left\langle p_{1}\left|: \widetilde{\phi}_{0}\left(k_{2}\right) \widetilde{\phi}_{0}\left(q_{2}\right):\right| p_{2}\right\rangle \\
= & \cos \left(\frac{k_{1} \wedge k_{2}}{2}\right) \cos \left(\frac{q_{1} \wedge q_{2}}{2}\right) \widetilde{\phi}_{0}^{c_{1}}\left(k_{1}\right) \widetilde{\phi}_{0}^{c_{1}}\left(q_{1}\right) \widetilde{\phi}_{0}^{c_{2}}\left(k_{3}-\omega\right) \widetilde{\phi}_{0}^{c_{2}}\left(q_{3}+\omega\right) \\
\times & \left\langle p_{1}\left|: \widetilde{\phi}_{0}\left(k_{2}\right) \widetilde{\phi}_{0}\left(q_{2}\right):\right| p_{2}\right\rangle .
\end{aligned}
$$

Novamente usamos as funções delta para trocar os argumentos dos cosenos. Assim, verificamos que esse termo é igual ao primeiro termo em (4.15). Seguindo o mesmo raciocínio podemos mostrar que todos os termos são iguais, como mencionado. 
Retornemos à equação (4.7). De acordo com a discussão feita, podemos escrever,

$$
\begin{aligned}
\left\langle p_{1}\left|A_{2}\right| p_{2}\right\rangle & =-\left(\frac{g}{3 !}\right)^{2} \int \frac{d \omega}{2 \pi i} \frac{1}{\omega+i \epsilon} \prod_{i=1}^{3} \int \frac{d^{D} k_{i}}{(2 \pi)^{D}} \prod_{j=1}^{3} \int \frac{d^{D} q_{j}}{(2 \pi)^{D}} \\
& \times(2 \pi)^{D} \delta^{D}\left(k_{1}+k_{2}+k_{3}\right) \cos \left(\frac{k_{1} \wedge k_{2}}{2}\right)(2 \pi)^{D} \delta^{D}\left(q_{1}+q_{2}+q_{3}\right) \cos \left(\frac{q_{1} \wedge q_{2}}{2}\right) \\
& \times(3 !)^{2}(2 \pi)^{D} \delta^{D}\left(k_{1}+q_{1}\right) \widetilde{\Delta}^{+}\left(k_{1}\right)(2 \pi)^{D} \delta^{D}\left(k_{3}+q_{3}\right) \widetilde{\Delta}^{+}\left(k_{3}-\omega\right) \\
& \times\left\langle p_{1}\left|: \widetilde{\phi}_{0}\left(k_{2}\right) \widetilde{\phi}_{0}\left(q_{2}\right):\right| p_{2}\right\rangle .
\end{aligned}
$$

Ainda falta o cálculo do elemento de matriz na última linha. Para esse fim, necessitamos da forma explícita do campo escalar;

$$
\phi(x)=\frac{1}{(2 \pi)^{\frac{D-1}{2}}} \int \frac{d^{D-1} q}{2 \omega_{q}}\left[e^{-i q x} a_{q}+e^{i q x} a_{q}^{\dagger}\right] .
$$

Os operadores de destruição $a_{q}$ e de criação $a_{q}^{\dagger}$ satisfazem a relação de comutação:

$$
\left[a_{q}, a_{p}^{\dagger}\right]=2 \omega_{q} \delta^{D-1}(\mathbf{q}-\mathbf{p}) .
$$

Calculando o elemento de matriz,

$$
\begin{aligned}
& \left\langle p_{1}\left|: \widetilde{\phi}_{0}\left(k_{2}\right) \widetilde{\phi}_{0}\left(q_{2}\right):\right| p_{2}\right\rangle \\
= & \int d^{D} z_{1} d^{D} z_{2} e^{i k_{2} z_{1}} e^{i q_{2} z_{2}}\left\langle p_{1}\left|: \phi_{0}\left(z_{1}\right) \phi_{0}\left(z_{2}\right):\right| p_{2}\right\rangle \\
= & \int d^{D} z_{1} d^{D} z_{2} e^{i k_{2} z_{1}} e^{i q_{2} z_{2}} \frac{1}{(2 \pi)^{D-1}} \int \frac{d^{D-1} l_{1}}{2 \omega_{l_{1}}} \int \frac{d^{D-1} l_{2}}{2 \omega_{l_{2}}} \\
\times & \langle 0| a_{p_{1}}\left(e^{-i l_{1} z_{1}-i l_{2} z_{2}} a_{l_{1}} a_{l_{2}}+e^{-i l_{1} z_{1}+i l_{2} z_{2}} a_{l_{2}}^{\dagger} a_{l_{1}}\right. \\
+ & \left.e^{i l_{1} z_{1}-i l_{2} z_{2}} a_{l_{1}}^{\dagger} a_{l_{2}}+e^{i l_{1} z_{1}+i l_{2} z_{2}} a_{l_{1}}^{\dagger} a_{l_{2}}^{\dagger}\right) a_{p_{2}}^{\dagger}|0\rangle \\
= & \int d^{D} z_{1} d^{D} z_{2} e^{i k_{2} z_{1}} e^{i q_{2} z_{2}} \frac{1}{(2 \pi)^{D-1}} \int \frac{d^{D-1} l_{1}}{2 \omega_{l_{1}}} \int \frac{d^{D-1} l_{2}}{2 \omega_{l_{2}}} \\
\times & {\left[e^{-i l_{1} z_{1}+i l_{2} z_{2}} 2 \omega_{p_{1}} \delta^{D-1}\left(\mathbf{p}_{1}-\mathbf{l}_{2}\right) 2 \omega_{l_{1}} \delta^{D-1}\left(\mathbf{l}_{1}-\mathbf{p}_{2}\right)\right.} \\
+ & \left.e^{i l_{1} z_{1}-i l_{2} z_{2}} 2 \omega_{p_{1}} \delta^{D-1}\left(\mathbf{p}_{1}-\mathbf{l}_{1}\right) 2 \omega_{l_{2}} \delta^{D-1}\left(\mathbf{l}_{2}-\mathbf{p}_{2}\right)\right] \\
= & \frac{1}{(2 \pi)^{D-1}}\left[(2 \pi)^{D} \delta^{D}\left(k_{2}-p_{2}\right)(2 \pi)^{D} \delta^{D}\left(q_{2}+p_{1}\right)\right. \\
+ & \left.(2 \pi)^{D} \delta^{D}\left(k_{2}+p_{1}\right)(2 \pi)^{D} \delta^{D}\left(q_{2}-p_{2}\right)\right]
\end{aligned}
$$


Levando esse resultado em (4.20), ficamos com

$$
\begin{aligned}
\left\langle p_{1}\left|A_{2}\right| p_{2}\right\rangle & =-\frac{g^{2}}{(2 \pi)^{D-1}} \int \frac{d \omega}{2 \pi i} \frac{1}{\omega+i \epsilon} \prod_{i=1}^{3} \int \frac{d^{D} k_{i}}{(2 \pi)^{D}} \prod_{j=1}^{3} \int \frac{d^{D} q_{j}}{(2 \pi)^{D}} \\
& \times(2 \pi)^{D} \delta^{D}\left(k_{1}+k_{2}+k_{3}\right) \cos \left(\frac{k_{1} \wedge k_{2}}{2}\right)(2 \pi)^{D} \delta^{D}\left(q_{1}+q_{2}+q_{3}\right) \cos \left(\frac{q_{1} \wedge q_{2}}{2}\right) \\
& \times(2 \pi)^{D} \delta^{D}\left(k_{1}+q_{1}\right) \widetilde{\Delta}^{+}\left(k_{1}\right)(2 \pi)^{D} \delta^{D}\left(k_{3}+q_{3}\right) \widetilde{\Delta}^{+}\left(k_{3}-\omega\right) \\
& \times\left[(2 \pi)^{D} \delta^{D}\left(k_{2}-p_{2}\right)(2 \pi)^{D} \delta^{D}\left(q_{2}+p_{1}\right)\right. \\
& \left.+(2 \pi)^{D} \delta^{D}\left(k_{2}+p_{1}\right)(2 \pi)^{D} \delta^{D}\left(q_{2}-p_{2}\right)\right] .
\end{aligned}
$$

Em primeiro lugar, calculamos as integrais em $q_{1}$ e $q_{3}$ :

$$
\begin{aligned}
\left\langle p_{1}\left|A_{2}\right| p_{2}\right\rangle & =-\frac{g^{2}}{(2 \pi)^{D-1}} \int \frac{d \omega}{2 \pi i} \frac{1}{\omega+i \epsilon} \prod_{i=1}^{3} \int \frac{d^{D} k_{i}}{(2 \pi)^{D}} \int \frac{d^{D} q_{2}}{(2 \pi)^{D}} \\
& \times(2 \pi)^{D} \delta^{D}\left(k_{1}+k_{2}+k_{3}\right) \cos \left(\frac{k_{1} \wedge k_{2}}{2}\right)(2 \pi)^{D} \delta^{D}\left(-k_{1}+q_{2}-k_{3}\right) \\
& \times \cos \left(\frac{k_{1} \wedge q_{2}}{2}\right) \widetilde{\Delta}^{+}\left(k_{1}\right) \widetilde{\Delta}^{+}\left(k_{3}-\omega\right) \\
& \times\left[(2 \pi)^{D} \delta^{D}\left(k_{2}-p_{2}\right)(2 \pi)^{D} \delta^{D}\left(q_{2}+p_{1}\right)\right. \\
& \left.+(2 \pi)^{D} \delta^{D}\left(k_{2}+p_{1}\right)(2 \pi)^{D} \delta^{D}\left(q_{2}-p_{2}\right)\right] .
\end{aligned}
$$

Na sequência, a integral em $q_{2}$;

$$
\begin{aligned}
\left\langle p_{1}\left|A_{2}\right| p_{2}\right\rangle & =-\frac{g^{2}}{(2 \pi)^{D-1}} \int \frac{d \omega}{2 \pi i} \frac{1}{\omega+i \epsilon} \prod_{i=1}^{3} \int \frac{d^{D} k_{i}}{(2 \pi)^{D}} \\
& \times(2 \pi)^{D} \delta^{D}\left(k_{1}+k_{2}+k_{3}\right) \cos ^{2}\left(\frac{k_{1} \wedge k_{2}}{2}\right) \widetilde{\Delta}^{+}\left(k_{1}\right) \widetilde{\Delta}^{+}\left(k_{3}-\omega\right) \\
& \times\left[(2 \pi)^{D} \delta^{D}\left(k_{2}-p_{2}\right)(2 \pi)^{D} \delta^{D}\left(k_{1}+k_{3}+p_{1}\right)\right. \\
& \left.+(2 \pi)^{D} \delta^{D}\left(k_{2}+p_{1}\right)(2 \pi)^{D} \delta^{D}\left(k_{1}+k_{3}-p_{2}\right)\right]
\end{aligned}
$$

Integral em $k_{3}$, 


$$
\begin{aligned}
\left\langle p_{1}\left|A_{2}\right| p_{2}\right\rangle & =-\frac{g^{2}}{(2 \pi)^{D-1}} \int \frac{d \omega}{2 \pi i} \frac{1}{\omega+i \epsilon} \int \frac{d^{D} k_{1}}{(2 \pi)^{D}} \int \frac{d^{D} k_{2}}{(2 \pi)^{D}} \\
& \times \cos ^{2}\left(\frac{k_{1} \wedge k_{2}}{2}\right) \widetilde{\Delta}^{+}\left(k_{1}\right) \widetilde{\Delta}^{+}\left(-k_{1}-k_{2}-\omega\right) \\
& \times\left[(2 \pi)^{D} \delta^{D}\left(k_{2}-p_{2}\right)(2 \pi)^{D} \delta^{D}\left(-k_{2}+p_{1}\right)\right. \\
& \left.+(2 \pi)^{D} \delta^{D}\left(k_{2}+p_{1}\right)(2 \pi)^{D} \delta^{D}\left(k_{2}+p_{2}\right)\right] .
\end{aligned}
$$

Por fim, calculamos a integral em $k_{2}$;

$$
\begin{aligned}
\left\langle p_{1}\left|A_{2}\right| p_{2}\right\rangle & =-(2 \pi)^{D} \delta^{D}\left(p_{2}-p_{1}\right) \frac{g^{2}}{(2 \pi)^{D-1}} \int \frac{d \omega}{2 \pi i} \frac{1}{\omega+i \epsilon} \int \frac{d^{D} k_{1}}{(2 \pi)^{D}} \\
& \times\left[\cos ^{2}\left(\frac{k_{1} \wedge p_{2}}{2}\right) \widetilde{\Delta}^{+}\left(k_{1}\right) \widetilde{\Delta}^{+}\left(-k_{1}-p_{2}-\omega\right)\right. \\
& \left.+\cos ^{2}\left(\frac{k_{1} \wedge p_{1}}{2}\right) \widetilde{\Delta}^{+}\left(k_{1}\right) \widetilde{\Delta}^{+}\left(-k_{1}+p_{1}-\omega\right)\right] .
\end{aligned}
$$

Agora, vamos analisar o lado esquerdo da relação de unitariedade (4.2),

$$
\begin{aligned}
\left\langle p_{1}\left|A_{2}+A_{2}^{\dagger}\right| p_{2}\right\rangle & =\left\langle p_{1}\left|A_{2}\right| p_{2}\right\rangle+\left\langle p_{2}\left|A_{2}\right| p_{1}\right\rangle^{*} \\
& =\left\langle p_{1}\left|A_{2}\right| p_{2}\right\rangle+\left\langle p_{1}\left|A_{2}\right| p_{2}\right\rangle^{*} \\
& =2 \operatorname{Re}\left\langle p_{1}\left|A_{2}\right| p_{2}\right\rangle .
\end{aligned}
$$

Para obter segunda linha usamos a simetria $p_{1} \leftrightarrow p_{2}$ da amplitude (4.28), que existe devido à delta de conservação de momento. Caso não houvesse essa simetria, não teríamos a parte real. Sendo assim, devemos extrair a parte real de (4.28). Isso é feito com auxílio da identidade

$$
\frac{1}{\omega+i \epsilon}=\mathrm{P}\left(\frac{1}{\omega}\right)-i \pi \delta(\omega),
$$

com P designando o valor principal de Cauchy. Quando usamos essa identidade em (4.28), esta separa-se diretamente em uma parte real e uma parte imaginária, sendo a parte real dada por, 


$$
\begin{aligned}
\operatorname{Re}\left\langle p_{1}\left|A_{2}\right| p_{2}\right\rangle & =(2 \pi)^{D} \delta^{D}\left(p_{2}-p_{1}\right) \frac{1}{2} \frac{g^{2}}{(2 \pi)^{D-1}} \int \frac{d^{D} k_{1}}{(2 \pi)^{D}} \\
& \times\left[\cos ^{2}\left(\frac{k_{1} \wedge p_{2}}{2}\right) \widetilde{\Delta}^{+}\left(k_{1}\right) \widetilde{\Delta}^{+}\left(-k_{1}-p_{2}\right)\right. \\
& \left.+\cos ^{2}\left(\frac{k_{1} \wedge p_{1}}{2}\right) \widetilde{\Delta}^{+}\left(k_{1}\right) \widetilde{\Delta}^{+}\left(-k_{1}+p_{1}\right)\right] .
\end{aligned}
$$

Partimos agora para o cálculo do lado direito da condição de unitariedade (4.2).

$$
\begin{aligned}
\left\langle p_{1}\left|A_{1}^{\dagger} A_{1}\right| p_{2}\right\rangle & =\left\langle p_{1}\left|\int d^{D} x d^{D} y \mathcal{F}_{x y}\left[\mathcal{V}\left(\phi_{0}(x)\right) \mathcal{V}\left(\phi_{0}(y)\right)\right]\right| p_{2}\right\rangle \\
& =\left(\frac{g}{3 !}\right)^{2} \int d^{D} x d^{D} y\left\langle p_{1}\left|\mathcal{F}_{x y}\left[\phi_{0}^{3}(x) \phi_{0}^{3}(y)\right]\right| p_{2}\right\rangle \\
& =\left(\frac{g}{3 !}\right)^{2} \int d^{D} x d^{D} y\left\langle p_{1}\left|\left(\phi_{0}(x) * \phi_{0}(x) * \phi_{0}(x)\right)\left(\phi_{0}(y) * \phi_{0}(y) * \phi_{0}(y)\right)\right| p_{2}\right\rangle .
\end{aligned}
$$

Os procedimentos para calcular a amplitude acima são os mesmo feitos no caso anterior, com a simplificação adicional devido a ausência do ordenamento temporal dado pela função $\theta$. Então, usamos (1.45) para escrever (4.32) como,

$$
\begin{aligned}
\left\langle p_{1}\left|A_{1}^{\dagger} A_{1}\right| p_{2}\right\rangle & =\left(\frac{g}{3 !}\right)^{2} \prod_{i=1}^{3} \int \frac{d^{D} k_{i}}{(2 \pi)^{D}} \prod_{j=1}^{3} \int \frac{d^{D} q_{j}}{(2 \pi)^{D}}(2 \pi)^{D} \delta^{D}\left(k_{1}+k_{2}+k_{3}\right) \cos \left(\frac{k_{1} \wedge k_{2}}{2}\right) \\
& \times(2 \pi)^{D} \delta^{D}\left(q_{1}+q_{2}+q_{3}\right) \cos \left(\frac{q_{1} \wedge q_{2}}{2}\right) \\
& \times\left\langle p_{1}\left|\widetilde{\phi}_{0}\left(k_{1}\right) \widetilde{\phi}_{0}\left(k_{2}\right) \widetilde{\phi}_{0}\left(k_{3}\right) \widetilde{\phi}_{0}\left(q_{1}\right) \widetilde{\phi}_{0}\left(q_{2}\right) \widetilde{\phi}_{0}\left(q_{3}\right)\right| p_{2}\right\rangle .
\end{aligned}
$$

Para calcular o elemento de matriz aplicamos o teorema de Wick, selecionando os termos que contribuem para o diagrama da figura (4.1). Como anteriormente, teremos (3!) ${ }^{2}$ termos que dão a mesma contribuição para a amplitude (4.33), ou seja,

$$
\begin{aligned}
\left\langle p_{1}\left|A_{1}^{\dagger} A_{1}\right| p_{2}\right\rangle & =\left(\frac{g}{3 !}\right)^{2} \prod_{i=1}^{3} \int \frac{d^{D} k_{i}}{(2 \pi)^{D}} \prod_{j=1}^{3} \int \frac{d^{D} q_{j}}{(2 \pi)^{D}}(2 \pi)^{D} \delta^{D}\left(k_{1}+k_{2}+k_{3}\right) \cos \left(\frac{k_{1} \wedge k_{2}}{2}\right) \\
& \times(2 \pi)^{D} \delta^{D}\left(q_{1}+q_{2}+q_{3}\right) \cos \left(\frac{q_{1} \wedge q_{2}}{2}\right) \\
& \times(3 !)^{2}(2 \pi)^{D} \delta^{D}\left(k_{1}+q_{1}\right) \widetilde{\Delta}^{+}\left(k_{1}\right)(2 \pi)^{D} \delta^{D}\left(k_{2}+q_{2}\right) \widetilde{\Delta}^{+}\left(k_{2}\right) \\
& \times\left\langle p_{1}\left|: \widetilde{\phi}_{0}\left(k_{3}\right) \widetilde{\phi}_{0}\left(q_{3}\right):\right| p_{2}\right\rangle .
\end{aligned}
$$


Esse último elemento de matriz foi calculado em (4.23). De acordo com esse resultado, segue que

$$
\begin{aligned}
\left\langle p_{1}\left|A_{1}^{\dagger} A_{1}\right| p_{2}\right\rangle & =\frac{g^{2}}{(2 \pi)^{D-1}} \prod_{i=1}^{3} \int \frac{d^{D} k_{i}}{(2 \pi)^{D}} \prod_{j=1}^{3} \int \frac{d^{D} q_{j}}{(2 \pi)^{D}}(2 \pi)^{D} \delta^{D}\left(k_{1}+k_{2}+k_{3}\right) \cos \left(\frac{k_{1} \wedge k_{2}}{2}\right) \\
& \times(2 \pi)^{D} \delta^{D}\left(q_{1}+q_{2}+q_{3}\right) \cos \left(\frac{q_{1} \wedge q_{2}}{2}\right) \\
& \times(2 \pi)^{D} \delta^{D}\left(k_{1}+q_{1}\right) \widetilde{\Delta}^{+}\left(k_{1}\right)(2 \pi)^{D} \delta^{D}\left(k_{2}+q_{2}\right) \widetilde{\Delta}^{+}\left(k_{2}\right) \\
& \times\left[(2 \pi)^{D} \delta^{D}\left(k_{3}-p_{2}\right)(2 \pi)^{D} \delta^{D}\left(q_{3}+p_{1}\right)\right. \\
& \left.+(2 \pi)^{D} \delta^{D}\left(k_{3}+p_{1}\right)(2 \pi)^{D} \delta^{D}\left(q_{3}-p_{2}\right)\right] .
\end{aligned}
$$

Vamos ao cálculo das integrais. Primeiramente, as integrais em $q_{1}$ e $q_{2}$;

$$
\begin{aligned}
\left\langle p_{1}\left|A_{1}^{\dagger} A_{1}\right| p_{2}\right\rangle & =\frac{g^{2}}{(2 \pi)^{D-1}} \prod_{i=1}^{3} \int \frac{d^{D} k_{i}}{(2 \pi)^{D}} \int \frac{d^{D} q_{3}}{(2 \pi)^{D}}(2 \pi)^{D} \delta^{D}\left(k_{1}+k_{2}+k_{3}\right) \\
& \times(2 \pi)^{D} \delta^{D}\left(-k_{1}-k_{2}+q_{3}\right) \cos ^{2}\left(\frac{k_{1} \wedge k_{2}}{2}\right) \widetilde{\Delta}^{+}\left(k_{1}\right) \widetilde{\Delta}^{+}\left(k_{2}\right) \\
& \times\left[(2 \pi)^{D} \delta^{D}\left(k_{3}-p_{2}\right)(2 \pi)^{D} \delta^{D}\left(q_{3}+p_{1}\right)\right. \\
& \left.+(2 \pi)^{D} \delta^{D}\left(k_{3}+p_{1}\right)(2 \pi)^{D} \delta^{D}\left(q_{3}-p_{2}\right)\right] .
\end{aligned}
$$

Integral em $q_{3}$;

$$
\begin{aligned}
\left\langle p_{1}\left|A_{1}^{\dagger} A_{1}\right| p_{2}\right\rangle & =\frac{g^{2}}{(2 \pi)^{D-1}} \prod_{i=1}^{3} \int \frac{d^{D} k_{i}}{(2 \pi)^{D}}(2 \pi)^{D} \delta^{D}\left(k_{1}+k_{2}+k_{3}\right) \cos ^{2}\left(\frac{k_{1} \wedge k_{2}}{2}\right) \\
& \times \widetilde{\Delta}^{+}\left(k_{1}\right) \widetilde{\Delta}^{+}\left(k_{2}\right)\left[(2 \pi)^{D} \delta^{D}\left(k_{3}-p_{2}\right)(2 \pi)^{D} \delta^{D}\left(k_{1}+k_{2}+p_{1}\right)\right. \\
& \left.+(2 \pi)^{D} \delta^{D}\left(k_{3}+p_{1}\right)(2 \pi)^{D} \delta^{D}\left(k_{1}+k_{2}-p_{2}\right)\right] .
\end{aligned}
$$

Integral em $k_{3}$;

$$
\begin{aligned}
\left\langle p_{1}\left|A_{1}^{\dagger} A_{1}\right| p_{2}\right\rangle & =\frac{g^{2}}{(2 \pi)^{D-1}} \int \frac{d^{D} k_{1}}{(2 \pi)^{D}} \int \frac{d^{D} k_{2}}{(2 \pi)^{D}} \cos ^{2}\left(\frac{k_{1} \wedge k_{2}}{2}\right) \widetilde{\Delta}^{+}\left(k_{1}\right) \widetilde{\Delta}^{+}\left(k_{2}\right) \\
& \times\left[(2 \pi)^{D} \delta^{D}\left(-k_{1}-k_{2}-p_{2}\right)(2 \pi)^{D} \delta^{D}\left(k_{1}+k_{2}+p_{1}\right)\right. \\
& \left.+(2 \pi)^{D} \delta^{D}\left(-k_{1}-k_{2}+p_{1}\right)(2 \pi)^{D} \delta^{D}\left(k_{1}+k_{2}-p_{2}\right)\right] .
\end{aligned}
$$


Por último, calculamos a integral em $k_{2}$;

$$
\begin{aligned}
\left\langle p_{1}\left|A_{1}^{\dagger} A_{1}\right| p_{2}\right\rangle & =(2 \pi)^{D} \delta^{D}\left(p_{2}-p_{1}\right) \frac{g^{2}}{(2 \pi)^{D-1}} \int \frac{d^{D} k_{1}}{(2 \pi)^{D}} \\
& \times\left[\cos ^{2}\left(\frac{k_{1} \wedge p_{2}}{2}\right) \widetilde{\Delta}^{+}\left(k_{1}\right) \widetilde{\Delta}^{+}\left(-k_{1}-p_{2}\right)\right. \\
& \left.+\cos ^{2}\left(\frac{k_{1} \wedge p_{1}}{2}\right) \widetilde{\Delta}^{+}\left(k_{1}\right) \widetilde{\Delta}^{+}\left(-k_{1}+p_{1}\right)\right] .
\end{aligned}
$$

Comparando esse resultado com (4.31), vemos que a condição de unitariedade em segunda ordem na constante de acoplamento,

$$
2 \operatorname{Re}\left\langle p_{1}\left|A_{2}\right| p_{2}\right\rangle=\left\langle p_{1}\left|A_{1}^{\dagger} A_{1}\right| p_{2}\right\rangle,
$$

é satisfeita. Esse é o resultado que esperávamos, resolvendo assim o problema da unitariedade no modelo $\phi^{3}$ para o diagrama de um laço e de segunda ordem na constante de acoplamento, que havia apresentado problema quando analisado dentro contexto do segundo capítulo.

Todos os estudos até aqui têm sido feitos com campos escalares. A sequência natural é estender a análise anterior para o caso de campos fermiônicos. No capítulo seguinte estudaremos o modelo de Yukawa que, além do campo escalar, envolve campos fermiônicos. 


\section{Capítulo 5}

\section{Modelo de Yukawa}

Para finalizar esse trabalho, investigaremos o modelo de Yukawa, cuja lagrangiana de interação envolve dois campos fermiônicos e um campo escalar. A escolha desse modelo se deve, por um lado, que em alguns aspectos ele é semelhante ao modelo $\phi^{3}$. Por outro lado, a presença de campos fermiônicos requer modificações. Portanto, a verificação da unitariedade nesse modelo reforça a consistência do método apresentado no terceiro capítulo.

\subsection{Diagrama com pernas externas fermiônicas}

A densidade de lagrangiana do modelo de Yukawa é dada por

$$
\mathcal{L}_{\text {Yukawa }}=\frac{1}{2} \bar{\psi}\left(i \gamma^{\mu} \partial_{\mu}-M\right) \psi+\frac{1}{2} \partial_{\mu} \phi \partial^{\mu} \phi-\frac{m^{2}}{2} \phi^{2}-\frac{\lambda}{2} \bar{\psi} * \psi * \phi .
$$

Nessa lagrangiana, $\psi$ e $\bar{\psi}$ são campos fermiônicos de Majorana, isto é, eles estão conectados pela matriz de conjugação de carga $C ; \psi=\bar{\psi} C$. A construção e algumas propriedades desses campos são discutidos no apêndice C. O motivo da escolha de campos de Majorana, ao invés de campos de Dirac, é que a não comutatividade, no último caso, não se manifesta em diagramas da ordem que queremos estudar, o que requer a análise de diagramas de ordens mais altas, aumentando a dificuldade do problema.

Assim como fizemos no modelo $\phi^{3}$, no capítulo anterior, investigaremos a unitariedade do modelo de Yukawa para processos específicos, quer dizer, para diagramas específicos. O primeiro diagrama que estudaremos é mostrado na figura (5.1). Nele, linhas pontilhadas são linhas fermiônicas e a linha contínua é uma linha bosônica. Devemos mencionar que a análise desse diagrama seguindo o tratamento do segundo capítulo também conduziria a uma quebra de unitariedade quando $\Theta_{0 i} \neq 0$. 


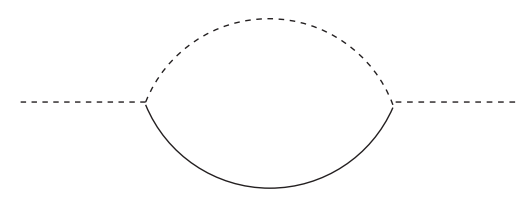

Figura 5.1: Diagrama de um laço com pernas externas fermiônicas

Como o diagrama considerado é de segunda ordem na constante de acoplamento, a relação de unitariedade que estudaremos é a mesma que anteriormente;

$$
\left\langle p_{1}\left|A_{2}+A_{2}^{\dagger}\right| p_{2}\right\rangle=\left\langle p_{1}\left|A_{1}^{\dagger} A_{1}\right| p_{2}\right\rangle .
$$

Os estados $\left|p_{1}\right\rangle$ e $\left|p_{2}\right\rangle$ ainda têm polarizações associadas, dizemos $t_{1}$ e $t_{2}$, respectivamente, mas omitiremos essa informação no que segue e a retomaremos quando necessária. Deve ser notado que trocando os estados $\left|p_{1}\right\rangle$ e $\left|p_{2}\right\rangle$ por estados de partículas bosônicas existe ainda outro diagrama que contribui para a relação acima; um laço fermiônico com pernas externas bosônicas. Esse diagrama será investigado na seção seguinte. O procedimento de cálculo segue os mesmos passos feitos no estudo de $\phi^{3}$, levando em conta, obviamente, o caráter fermiônico dos campos. Inicialmente, consideremos a amplitude $\left\langle p_{1}\left|A_{2}\right| p_{2}\right\rangle$ :

$$
\left\langle p_{1}\left|A_{2}\right| p_{2}\right\rangle=\left(\frac{\lambda}{2}\right)^{2} \int d^{D} x d^{D} y\left\langle p_{1}\left|\mathcal{F}_{x y}\left[\theta\left(x^{0}-y^{0}\right) \bar{\psi}_{\alpha}(x) \psi_{\alpha}(x) \phi(x) \bar{\psi}_{\gamma}(y) \psi_{\gamma}(y) \phi(y)\right]\right| p_{2}\right\rangle
$$

Dessa expressão em diante omitiremos o índice zero que servia para indicar campo livre. Usando a representação integral para a função $\theta$, equação (4.4), podemos escrever

$$
\begin{aligned}
\left\langle p_{1}\left|A_{2}\right| p_{2}\right\rangle & =-\frac{\lambda^{2}}{4} \int d^{D} x d^{D} y \int \frac{d \omega}{2 \pi i} \frac{1}{\omega+i \epsilon} \\
& \times\left\langle p_{1}\left|\mathcal{F}_{x y}\left[e^{-i \omega\left(x_{i}^{0}-y_{j}^{0}\right)} \bar{\psi}_{\alpha}\left(x_{1}\right) \psi_{\alpha}\left(x_{2}\right) \phi\left(x_{3}\right) \bar{\psi}_{\gamma}\left(y_{1}\right) \psi_{\gamma}\left(y_{2}\right) \phi\left(y_{3}\right)\right]\right| p_{2}\right\rangle .
\end{aligned}
$$

Aqui, devemos examinar a realização minimal com maior detalhe. Nesse procedimento, incorporamos a função $\theta$ em campos que estão contraídos. Então, por conveniência, escolhemos incorporar as exponenciais oriundas da função $\theta$ aos campos escalares, que deverão estar contraídos nos termos que contribuem para o diagrama (5.1). As contrações serão feitas posteriormente na aplicação de teorema de Wick. Segue que, 


$$
\begin{aligned}
\left\langle p_{1}\left|A_{2}\right| p_{2}\right\rangle & =-\frac{\lambda^{2}}{4} \int d^{D} x d^{D} y \int \frac{d \omega}{2 \pi i} \frac{1}{\omega+i \epsilon} \\
& \times\left\langle p_{1}\left|\left(\bar{\psi}_{\alpha}(x) * \psi_{\alpha}(x) * \varphi(x)\right)\left(\bar{\psi}_{\gamma}(y) * \psi_{\gamma}(y) * \chi(y)\right)\right| p_{2}\right\rangle,
\end{aligned}
$$

$\operatorname{com} \varphi(x) \equiv e^{-i \omega x} \phi(x)$ e $\chi(x) \equiv e^{i \omega y} \phi(y)$, tal que definimos o quadrivetor $\omega^{\mu} \equiv(\omega, 0,0,0)$. De acordo com a propriedade

$$
\begin{aligned}
\int d^{D} x \bar{\psi}_{\alpha}(x) * \psi_{\alpha}(x) * \phi(x) & =\int \frac{d^{D} k_{1}}{(2 \pi)^{D}} \frac{d^{D} k_{2}}{(2 \pi)^{D}} \frac{d^{D} k_{3}}{(2 \pi)^{D}}(2 \pi)^{D} \delta\left(k_{1}+k_{2}+k_{3}\right) \\
& \times \cos \left(\frac{k_{1} \wedge k_{2}}{2}\right) \widetilde{\bar{\psi}}_{\alpha}\left(k_{1}\right) \widetilde{\psi}_{\alpha}\left(k_{2}\right) \widetilde{\phi}\left(k_{3}\right),
\end{aligned}
$$

que é demonstrada no apêndice D, a equação (5.5) torna-se,

$$
\begin{aligned}
\left\langle p_{1}\left|A_{2}\right| p_{2}\right\rangle & =-\frac{\lambda^{2}}{4} \int \frac{d \omega}{2 \pi i} \frac{1}{\omega+i \epsilon} \prod_{i=1}^{3} \int \frac{d^{D} k_{i}}{(2 \pi)^{D}} \prod_{j=1}^{3} \int \frac{d^{D} q_{j}}{(2 \pi)^{D}} \\
& \times(2 \pi)^{D} \delta\left(k_{1}+k_{2}+k_{3}\right) \cos \left(\frac{k_{1} \wedge k_{2}}{2}\right)(2 \pi)^{D} \delta\left(q_{1}+q_{2}+q_{3}\right) \cos \left(\frac{q_{1} \wedge q_{2}}{2}\right) \\
& \times\left\langle p_{1}\left|\widetilde{\bar{\psi}}_{\alpha}\left(k_{1}\right) \widetilde{\psi}_{\alpha}\left(k_{2}\right) \widetilde{\varphi}\left(k_{3}\right) \widetilde{\bar{\psi}}_{\gamma}\left(q_{1}\right) \widetilde{\psi}_{\gamma}\left(q_{2}\right) \widetilde{\chi}\left(q_{3}\right)\right| p_{2}\right\rangle .
\end{aligned}
$$

Notemos que $\widetilde{\varphi}\left(k_{3}\right)=\widetilde{\phi}\left(k_{3}-\omega\right)$ e $\widetilde{\chi}\left(q_{3}\right)=\widetilde{\phi}\left(q_{3}+\omega\right)$. Devemos então calcular o elemento de matriz

$$
\left\langle p_{1}\left|\widetilde{\bar{\psi}}_{\alpha}\left(k_{1}\right) \widetilde{\psi}_{\alpha}\left(k_{2}\right) \widetilde{\phi}\left(k_{3}-\omega\right) \widetilde{\bar{\psi}}_{\gamma}\left(q_{1}\right) \widetilde{\psi}_{\gamma}\left(q_{2}\right) \widetilde{\phi}\left(q_{3}+\omega\right)\right| p_{2}\right\rangle,
$$

selecionando apenas os termos que contribuem para o diagrama em consideração. Isso é feito usando o teorema de Wick, que é essencialmente o mesmo apresentado em (4.11), porém levando em conta o caráter anti-comutante dos campos fermiônico envolvidos. Assim, o teorema de Wick para um produto ordinário de campos fermiônicos de Majorana é:

$$
\begin{aligned}
& \psi_{\alpha_{1}}\left(z_{1}\right) \psi_{\alpha_{2}}\left(z_{2}\right) \psi_{\alpha_{3}}\left(z_{3}\right) \cdots \psi_{\alpha_{N}}\left(z_{N}\right)=: \psi_{\alpha_{1}}\left(z_{1}\right) \psi_{\alpha_{2}}\left(z_{2}\right) \psi_{\alpha_{3}}\left(z_{3}\right) \cdots \psi_{\alpha_{N}}\left(z_{N}\right): \\
+\quad & : \psi_{\alpha_{1}}^{c_{1}}\left(z_{1}\right) \psi_{\alpha_{2}}^{c_{1}}\left(z_{2}\right) \psi_{\alpha_{3}}\left(z_{3}\right) \cdots \psi_{\alpha_{N}}\left(z_{N}\right):+: \psi_{\alpha_{1}}^{c_{1}}\left(z_{1}\right) \psi_{\alpha_{2}}\left(z_{2}\right) \psi_{\alpha_{3}}^{c_{1}}\left(z_{3}\right) \cdots \psi_{\alpha_{N}}\left(z_{N}\right):+\cdots \\
+ & : \psi_{\alpha_{1}}^{c_{1}}\left(z_{1}\right) \psi_{\alpha_{2}}^{c_{1}}\left(z_{2}\right) \psi_{\alpha_{3}}^{c_{2}}\left(z_{3}\right) \psi_{\alpha_{4}}^{c_{2}}\left(z_{4}\right) \cdots \psi_{\alpha_{N}}\left(z_{N}\right):+\cdots \\
+ & : \psi_{\alpha_{1}}^{c_{1}}\left(z_{1}\right) \psi_{\alpha_{2}}^{c_{1}}\left(z_{2}\right) \psi_{\alpha_{3}}^{c_{2}}\left(z_{3}\right) \psi_{\alpha_{4}}^{c_{2}}\left(z_{4}\right) \cdots \psi_{\alpha_{N-1}}^{c_{n}}\left(z_{N-1}\right) \psi_{\alpha_{N}}^{c_{n}}\left(z_{N}\right):
\end{aligned}
$$


Estamos usando a notação

$$
\begin{aligned}
& : \psi_{\alpha_{1}}\left(z_{1}\right) \cdots \psi_{\alpha_{i}}^{c_{1}}\left(z_{i}\right) \cdots \psi_{\alpha_{j}}^{c_{1}}\left(z_{j}\right) \cdots \psi_{\alpha_{N}}\left(z_{N}\right): \\
\equiv & (-1)^{P} \psi_{\alpha_{i}}^{c_{1}}\left(z_{i}\right) \psi_{\alpha_{j}}^{c_{1}}\left(z_{j}\right): \psi_{\alpha_{1}}\left(z_{1}\right) \cdots \hat{\psi}_{\alpha_{i}}\left(z_{i}\right) \cdots \hat{\psi}_{\alpha_{j}}\left(z_{j}\right) \cdots \psi_{\alpha_{N}}\left(z_{N}\right): .
\end{aligned}
$$

$\hat{\psi}_{\alpha}$ indica a ausência desse campo no produto e $P$ é o número de permutações entre campos fermiônicos para deixar um dos campos $\psi$ imediatamente ao lado de outro campo $\psi$ numa contração. Notemos que mesmo quando há um produto envolvendo campos $\psi$ e $\bar{\psi}$, podemos espressá-lo como um produto apenas entre campos $\psi$ ou apenas entre campos $\bar{\psi}$, bastando usar a relação $\psi=\bar{\psi} C$. A contração entre dois campos $\psi_{\alpha}$ é obtida a partir de

$$
\psi_{\alpha}^{c_{1}}\left(z_{1}\right) \bar{\psi}_{\beta}^{c_{1}}\left(z_{2}\right) \equiv\left\langle 0\left|\psi_{\alpha}\left(z_{1}\right) \bar{\psi}_{\beta}\left(z_{2}\right)\right| 0\right\rangle=S_{\alpha \beta}^{+}\left(z_{1}-z_{2}\right)
$$

com

$$
S_{\alpha \beta}^{+}\left(z_{1}-z_{2}\right)=\int \frac{d^{D} k}{(2 \pi)^{D}} e^{-i k\left(z_{1}-z_{2}\right)}\left(\gamma^{\mu} k_{\mu}+M\right)_{\alpha \beta}(2 \pi) \delta\left(k^{2}-M^{2}\right) \theta\left(k^{0}\right) .
$$

Com esse resultado, a contração entre dois campos $\psi_{\alpha}$ é dada por:

$$
\begin{aligned}
\psi_{\alpha}^{c_{1}}\left(z_{1}\right) \psi_{\beta}^{c_{1}}\left(z_{2}\right) & =\psi_{\alpha}^{c_{1}}\left(z_{1}\right) \bar{\psi}_{\gamma}^{c_{1}}\left(z_{2}\right) C_{\gamma \beta} \\
& =S_{\alpha \gamma}^{+}\left(z_{1}-z_{2}\right) C_{\gamma \beta} \\
& =\left[S^{+}\left(z_{1}-z_{2}\right) C\right]_{\alpha \beta}
\end{aligned}
$$

e entre dois campos $\bar{\psi}$ é

$$
\begin{aligned}
\bar{\psi}_{\alpha}^{c_{1}}\left(z_{1}\right) \bar{\psi}_{\beta}^{c_{1}}\left(z_{2}\right) & =C_{\gamma \alpha}^{-1} \psi_{\gamma}^{c_{1}}\left(z_{1}\right) \bar{\psi}_{\beta}^{c_{1}}\left(z_{2}\right) \\
& =C_{\gamma \alpha}^{-1} S_{\gamma \beta}^{+}\left(z_{1}-z_{2}\right) \\
& =\left[C_{\alpha \gamma}^{-1}\right]^{T} S_{\gamma \beta}^{+}\left(z_{1}-z_{2}\right) \\
& =-C_{\alpha \gamma}^{-1} S_{\gamma \beta}^{+}\left(z_{1}-z_{2}\right) \\
& =-\left[C^{-1} S^{+}\left(z_{1}-z_{2}\right)\right]_{\alpha \beta},
\end{aligned}
$$

visto que a matriz de conjugação de carga satisfaz $C=-C^{-1}=-C^{T}=-C^{\dagger}$. De forma análoga à equação (4.13), podemos obter uma contração no espaço de momentos:

$$
\widetilde{\psi}_{\alpha}^{c_{1}}\left(k_{1}\right) \widetilde{\bar{\psi}}_{\beta}^{c_{1}}\left(k_{2}\right)=(2 \pi)^{D} \delta^{D}\left(k_{1}+k_{2}\right) \widetilde{S}_{\alpha \beta}^{+}\left(k_{1}\right) .
$$


Agora podemos calcular o elemento de matriz (5.8). Em primeiro lugar, notemos que os termos que contribuem para o diagrama (5.1) são aqueles em que há apenas uma contração entre campos fermiônicos e uma contração entre os campos escalares. Além disso, na contração entre os campos fermiônicos, um dos campos deve ter o argumento pertencente ao conjunto $\left\{k_{1}, k_{2}\right\}$ e o outro pertencente ao conjunto $\left\{q_{1}, q_{2}\right\}$. Sendo assim, teremos

$$
\begin{aligned}
& \left\langle p_{1}\left|\widetilde{\bar{\psi}}_{\alpha}\left(k_{1}\right) \widetilde{\psi}_{\alpha}\left(k_{2}\right) \widetilde{\phi}\left(k_{3}-\omega\right) \widetilde{\bar{\psi}}_{\gamma}\left(q_{1}\right) \widetilde{\psi}_{\gamma}\left(q_{2}\right) \widetilde{\phi}\left(q_{3}+\omega\right)\right| p_{2}\right\rangle \\
= & -\widetilde{\bar{\psi}}_{\alpha}^{c_{1}}\left(k_{1}\right) \widetilde{\bar{\psi}}_{\gamma}^{c_{1}}\left(q_{1}\right) \widetilde{\phi}^{c_{2}}\left(k_{3}-\omega\right) \widetilde{\phi}^{c_{2}}\left(q_{3}+\omega\right)\left\langle p_{1}\left|: \widetilde{\psi}_{\alpha}\left(k_{2}\right) \widetilde{\psi}_{\gamma}\left(q_{2}\right):\right| p_{2}\right\rangle \\
+ & \widetilde{\bar{\psi}}_{\alpha}^{c_{1}}\left(k_{1}\right) \widetilde{\psi}_{\gamma}^{c_{1}}\left(q_{2}\right) \widetilde{\phi}^{c_{2}}\left(k_{3}-\omega\right) \widetilde{\phi}^{c_{2}}\left(q_{3}+\omega\right)\left\langle p_{1}\left|: \widetilde{\psi}_{\alpha}\left(k_{2}\right) \widetilde{\bar{\psi}}_{\gamma}\left(q_{1}\right):\right| p_{2}\right\rangle \\
+ & \widetilde{\psi}_{\alpha}^{c_{1}}\left(k_{2}\right) \widetilde{\bar{\psi}}_{\gamma}^{c_{1}}\left(q_{1}\right) \widetilde{\phi}^{c_{2}}\left(k_{3}-\omega\right) \widetilde{\phi}^{c_{2}}\left(q_{3}+\omega\right)\left\langle p_{1}\left|: \widetilde{\bar{\psi}}_{\alpha}\left(k_{1}\right) \widetilde{\psi}_{\gamma}\left(q_{2}\right):\right| p_{2}\right\rangle \\
- & \widetilde{\psi}_{\alpha}^{c_{1}}\left(k_{2}\right) \widetilde{\psi}_{\gamma}^{c_{1}}\left(q_{2}\right) \widetilde{\phi}^{c_{2}}\left(k_{3}-\omega\right) \widetilde{\phi}^{c_{2}}\left(q_{3}+\omega\right)\left\langle p_{1}\left|: \widetilde{\bar{\psi}}_{\alpha}\left(k_{1}\right) \widetilde{\bar{\psi}}_{\gamma}\left(q_{1}\right):\right| p_{2}\right\rangle .
\end{aligned}
$$

Por conveniência, queremos deixar os termos da soma da mesma forma que o terceiro termo, pois os campos fermiônicos contraídos nesse termo estão de tal modo que originam $\widetilde{S}_{\alpha \gamma}^{+}$, sem a presença da matriz de conjugação de carga. Faremos isso usando as relações $\psi_{\alpha}=\bar{\psi}_{\beta} C_{\beta \alpha}$ e $\bar{\psi}_{\alpha}=\psi_{\beta} C_{\beta \alpha}^{-1}$. Examinemos o primeiro termo:

$$
\begin{aligned}
& -\widetilde{\bar{\psi}}_{\alpha}^{c_{1}}\left(k_{1}\right) \widetilde{\bar{\psi}}_{\gamma}^{c_{1}}\left(q_{1}\right) \widetilde{\phi}^{c_{2}}\left(k_{3}-\omega\right) \widetilde{\phi}^{c_{2}}\left(q_{3}+\omega\right)\left\langle p_{1}\left|: \widetilde{\psi}_{\alpha}\left(k_{2}\right) \widetilde{\psi}_{\gamma}\left(q_{2}\right):\right| p_{2}\right\rangle \\
= & -\widetilde{\psi}_{\beta}^{c_{1}}\left(k_{1}\right) \widetilde{\bar{\psi}}_{\gamma}^{c_{1}}\left(q_{1}\right) \widetilde{\phi}^{c_{2}}\left(k_{3}-\omega\right) \widetilde{\phi}^{c_{2}}\left(q_{3}+\omega\right)\left\langle p_{1}\left|: C_{\beta \alpha}^{-1} \widetilde{\psi}_{\alpha}\left(k_{2}\right) \widetilde{\psi}_{\gamma}\left(q_{2}\right):\right| p_{2}\right\rangle \\
= & \widetilde{\psi}_{\beta}^{c_{1}}\left(k_{1}\right) \widetilde{\bar{\psi}}_{\gamma}^{c_{1}}\left(q_{1}\right) \widetilde{\phi}^{c_{2}}\left(k_{3}-\omega\right) \widetilde{\phi}^{c_{2}}\left(q_{3}+\omega\right)\left\langle p_{1}\left|: \widetilde{\bar{\psi}}_{\beta}\left(k_{2}\right) \widetilde{\psi}_{\gamma}\left(q_{2}\right):\right| p_{2}\right\rangle .
\end{aligned}
$$

Procedendo da mesma maneira podemos transformar o segundo e o quarto termo de modo que (5.16) torna-se,

$$
\begin{aligned}
& \left\langle p_{1}\left|\widetilde{\bar{\psi}}_{\alpha}\left(k_{1}\right) \widetilde{\psi}_{\alpha}\left(k_{2}\right) \widetilde{\phi}\left(k_{3}-\omega\right) \widetilde{\bar{\psi}}_{\gamma}\left(q_{1}\right) \widetilde{\psi}_{\gamma}\left(q_{2}\right) \widetilde{\phi}\left(q_{3}+\omega\right)\right| p_{2}\right\rangle \\
= & \widetilde{\psi}_{\alpha}^{c_{1}}\left(k_{1}\right) \widetilde{\bar{\psi}}_{\gamma}^{c_{1}}\left(q_{1}\right) \widetilde{\phi}^{c_{2}}\left(k_{3}-\omega\right) \widetilde{\phi}^{c_{2}}\left(q_{3}+\omega\right)\left\langle p_{1}\left|: \widetilde{\bar{\psi}}_{\alpha}\left(k_{2}\right) \widetilde{\psi}_{\gamma}\left(q_{2}\right):\right| p_{2}\right\rangle \\
+ & \widetilde{\psi}_{\alpha}^{c_{1}}\left(k_{1}\right) \widetilde{\bar{\psi}}_{\gamma}^{c_{1}}\left(q_{2}\right) \widetilde{\phi}^{c_{2}}\left(k_{3}-\omega\right) \widetilde{\phi}^{c_{2}}\left(q_{3}+\omega\right)\left\langle p_{1}\left|: \widetilde{\bar{\psi}}_{\alpha}\left(k_{2}\right) \widetilde{\psi}_{\gamma}\left(q_{1}\right):\right| p_{2}\right\rangle \\
+ & \widetilde{\psi}_{\alpha}^{c_{1}}\left(k_{2}\right) \widetilde{\bar{\psi}}_{\gamma}^{c_{1}}\left(q_{1}\right) \widetilde{\phi}^{c_{2}}\left(k_{3}-\omega\right) \widetilde{\phi}^{c_{2}}\left(q_{3}+\omega\right)\left\langle p_{1}\left|: \widetilde{\bar{\psi}}_{\alpha}\left(k_{1}\right) \widetilde{\psi}_{\gamma}\left(q_{2}\right):\right| p_{2}\right\rangle \\
+ & \widetilde{\psi}_{\alpha}^{c_{1}}\left(k_{2}\right) \widetilde{\bar{\psi}}_{\gamma}^{c_{1}}\left(q_{2}\right) \widetilde{\phi}^{c_{2}}\left(k_{3}-\omega\right) \widetilde{\phi}^{c_{2}}\left(q_{3}+\omega\right)\left\langle p_{1}\left|: \widetilde{\bar{\psi}}_{\alpha}\left(k_{1}\right) \widetilde{\psi}_{\gamma}\left(q_{1}\right):\right| p_{2}\right\rangle .
\end{aligned}
$$

Como todos os momentos estão integrados, podemos fazer mudanças de variáveis e verificar que todos esses termos são iguais. Consideremos o segundo termo: fazendo a mudança de variável $q_{1} \leftrightarrow q_{2}$ nele, ficamos com 


$$
\begin{aligned}
& \delta^{D}\left(q_{1}+q_{2}+q_{3}\right) \cos \left(\frac{q_{2} \wedge q_{1}}{2}\right) \widetilde{\psi}_{\alpha}^{c_{1}}\left(k_{1}\right) \widetilde{\bar{\psi}}_{\gamma}^{c_{1}}\left(q_{1}\right) \widetilde{\phi}^{c_{2}}\left(k_{3}-\omega\right) \widetilde{\phi}^{c_{2}}\left(q_{3}+\omega\right) \\
\times & \left\langle p_{1}\left|: \widetilde{\bar{\psi}}_{\alpha}\left(k_{2}\right) \widetilde{\psi}_{\gamma}\left(q_{2}\right):\right| p_{2}\right\rangle,
\end{aligned}
$$

que é igual ao primeiro termo. Portanto, escrevemos apenas um deles e multiplicamos por 4. Segue que,

$$
\begin{aligned}
\left\langle p_{1}\left|A_{2}\right| p_{2}\right\rangle & =-\frac{\lambda^{2}}{4} \int \frac{d \omega}{2 \pi i} \frac{1}{\omega+i \epsilon} \prod_{i=1}^{3} \int \frac{d^{D} k_{i}}{(2 \pi)^{D}} \prod_{j=1}^{3} \int \frac{d^{D} q_{j}}{(2 \pi)^{D}} \\
& \times(2 \pi)^{D} \delta\left(k_{1}+k_{2}+k_{3}\right) \cos \left(\frac{k_{1} \wedge k_{2}}{2}\right)(2 \pi)^{D} \delta\left(q_{1}+q_{2}+q_{3}\right) \cos \left(\frac{q_{1} \wedge q_{2}}{2}\right) \\
& \times 4(2 \pi)^{D} \delta^{D}\left(k_{1}+q_{1}\right) \widetilde{S}_{\alpha \gamma}^{+}\left(k_{1}\right)(2 \pi)^{D} \delta^{D}\left(k_{3}+q_{3}\right) \widetilde{\Delta}^{+}\left(k_{3}-\omega\right) \\
& \times\left\langle p_{1}\left|: \widetilde{\bar{\psi}}_{\alpha}\left(k_{2}\right) \widetilde{\psi}_{\gamma}\left(q_{2}\right):\right| p_{2}\right\rangle .
\end{aligned}
$$

Ainda nos resta o cálculo do elemento de matriz acima. Para esse fim, precisamos das forma explícitas dos campos de Majorana;

$$
\psi_{\alpha}(x)=\frac{1}{(2 \pi)^{\frac{D-1}{2}}} \sum_{r} \int \frac{d^{D-1} p}{\omega_{p} / M}\left[c_{p}^{(r)} u_{\alpha}^{(r)}(p) e^{-i p x}+c_{p}^{\dagger(r)} v_{\alpha}^{(r)}(p) e^{i p x}\right]
$$

$\mathrm{e}$

$$
\bar{\psi}_{\alpha}(x)=\frac{1}{(2 \pi)^{\frac{D-1}{2}}} \sum_{r} \int \frac{d^{D-1} p}{\omega_{p} / M}\left[c_{p}^{\dagger(r)} \bar{u}_{\alpha}^{(r)}(p) e^{i p x}+c_{p}^{(r)} \bar{v}_{\alpha}^{(r)}(p) e^{-i p x}\right] .
$$

Os operadores $c_{p}^{(r)}$ e $c_{p}^{\dagger(r)}$ satisfazem a relação de anti-comutação

$$
\left\{c_{p}^{(r)}, c_{q}^{\dagger(s)}\right\}=\frac{\omega_{p}}{M} \delta_{r s} \delta^{D-1}(\mathbf{p}-\mathbf{q})
$$

$u$ e $v$ são tais que

$$
\left(p_{\mu} \gamma^{\mu}-M\right) u(p)=0
$$

$\mathrm{e}$

$$
\left(p_{\mu} \gamma^{\mu}+M\right) v(p)=0 .
$$

Nessas equações a polarização $r$ foi omitida. A construção dos campos de Majorana a partir dos campos de Dirac é mostrada no apêndice C, como dito no início desta seção. Passemos ao cálculo do elemento de matriz remanescente; 


$$
\begin{aligned}
&\left\langle p_{1}\left|: \widetilde{\bar{\psi}}_{\alpha}\left(k_{2}\right) \widetilde{\psi}_{\gamma}\left(q_{2}\right):\right| p_{2}\right\rangle \\
&= \int d^{D} x d^{D} y e^{i k_{2} x} e^{i q_{2} y}\left\langle p_{1}\left|: \bar{\psi}_{\alpha}(x) \psi_{\gamma}(y):\right| p_{2}\right\rangle \\
&= \int d^{D} x d^{D} y e^{i k_{2} x} e^{i q_{2} y} \frac{1}{(2 \pi)^{D-1}} \sum_{s, r} \int \frac{d^{D-1} l_{1}}{\omega_{l_{1}} / M} \frac{d^{D-1} l_{2}}{\omega_{l_{2}} / M} \\
& \times\langle 0| c_{p_{1}}^{\left(t_{1}\right)}\left(\bar{u}_{\alpha}^{(r)}\left(l_{1}\right) u_{\gamma}^{(s)}\left(l_{2}\right) e^{i l_{1} x} e^{-i l_{2} y} c_{l_{1}}^{\dagger(r)} c_{l_{2}}^{(s)}\right. \\
&+ \bar{u}_{\alpha}^{(r)}\left(l_{1}\right) v_{\gamma}^{(s)}\left(l_{2}\right) e^{i l_{1} x} e^{i l_{2} y} c_{l_{1}}^{\dagger(r)} c_{l_{2}}^{\dagger(s)} \\
&+ \bar{v}_{\alpha}^{(r)}\left(l_{1}\right) u_{\gamma}^{(s)}\left(l_{2}\right) e^{-i l_{1} x} e^{-i l_{2} y} c_{l_{1}}^{(r)} c_{l_{2}}^{(s)} \\
&-\left.\bar{v}_{\alpha}^{(r)}\left(l_{1}\right) v_{\gamma}^{(s)}\left(l_{2}\right) e^{-i l_{1} x} e^{i l_{2} y} c_{l_{2}}^{\dagger(s)} c_{l_{1}}^{(r)}\right) c_{p_{2}}^{\dagger\left(t_{2}\right)}|0\rangle \\
&= \int d^{D} x d^{D} y e^{i k_{2} x} e^{i q_{2} y} \frac{1}{(2 \pi)^{D-1}} \sum_{s, r} \int \frac{d^{D-1} l_{1}}{\omega_{l_{1}} / M} \frac{d^{D-1} l_{2}}{\omega_{l_{2}} / M} \\
& \times {\left[\bar{u}_{\alpha}^{(r)}\left(l_{1}\right) u_{\gamma}^{(s)}\left(l_{2}\right) e^{i l_{1} x} e^{-i l_{2} y} \frac{\omega_{p_{1}}}{M} \delta_{t_{1} r} \delta^{D-1}\left(\mathbf{p}_{1}-\mathbf{l}_{1}\right)\right.} \\
& \times \frac{\omega_{l_{2}}}{M} \delta_{s t_{2}} \delta^{D-1}\left(\mathbf{l}_{2}-\mathbf{p}_{2}\right)-\bar{v}_{\alpha}^{(r)}\left(l_{1}\right) v_{\gamma}^{(s)}\left(l_{2}\right) e^{-i l_{1} x} e^{i l_{2} y} \\
& \times\left.\frac{\omega_{p_{1}}}{M} \delta_{t_{1} s} \delta^{D-1}\left(\mathbf{p}_{1}-\mathbf{l}_{2}\right) \frac{\omega_{l_{1}}}{M} \delta_{r t_{2}} \delta^{D-1}\left(\mathbf{l}_{1}-\mathbf{p}_{2}\right)\right] \\
&= \frac{1}{(2 \pi)^{D-1}}\left[\bar{u}_{\alpha}^{\left(t_{1}\right)}\left(p_{1}\right) u_{\gamma}^{\left(t_{2}\right)}\left(p_{2}\right)(2 \pi)^{D} \delta^{D}\left(k_{2}+p_{1}\right)(2 \pi)^{D} \delta^{D}\left(q_{2}-p_{2}\right)\right. \\
&-\left.\bar{v}_{\alpha}^{\left(t_{2}\right)}\left(p_{2}\right) v_{\gamma}^{\left(t_{1}\right)}\left(p_{1}\right)(2 \pi)^{D} \delta^{D}\left(k_{2}-p_{2}\right)(2 \pi)^{D} \delta^{D}\left(q_{2}+p_{1}\right)\right] . \\
&
\end{aligned}
$$

Deve ser notado que na ordenação de Wick de operadores fermiônicos temos um sinal negativo. Substituindo esse resultado em (5.20), obtemos,

$$
\begin{aligned}
\left\langle p_{1}\left|A_{2}\right| p_{2}\right\rangle & =-\frac{\lambda^{2}}{(2 \pi)^{D-1}} \int \frac{d \omega}{2 \pi i} \frac{1}{\omega+i \epsilon} \prod_{i=1}^{3} \int \frac{d^{D} k_{i}}{(2 \pi)^{D}} \prod_{j=1}^{3} \int \frac{d^{D} q_{j}}{(2 \pi)^{D}} \\
& \times(2 \pi)^{D} \delta\left(k_{1}+k_{2}+k_{3}\right) \cos \left(\frac{k_{1} \wedge k_{2}}{2}\right)(2 \pi)^{D} \delta\left(q_{1}+q_{2}+q_{3}\right) \cos \left(\frac{q_{1} \wedge q_{2}}{2}\right) \\
& \times(2 \pi)^{D} \delta^{D}\left(k_{1}+q_{1}\right) \widetilde{S}_{\alpha \gamma}^{+}\left(k_{1}\right)(2 \pi)^{D} \delta^{D}\left(k_{3}+q_{3}\right) \widetilde{\Delta}^{+}\left(k_{3}-\omega\right) \\
& \times\left[\bar{u}_{\alpha}^{\left(t_{1}\right)}\left(p_{1}\right) u_{\gamma}^{\left(t_{2}\right)}\left(p_{2}\right)(2 \pi)^{D} \delta^{D}\left(k_{2}+p_{1}\right)(2 \pi)^{D} \delta^{D}\left(q_{2}-p_{2}\right)\right. \\
& \left.-\bar{v}_{\alpha}^{\left(t_{2}\right)}\left(p_{2}\right) v_{\gamma}^{\left(t_{1}\right)}\left(p_{1}\right)(2 \pi)^{D} \delta^{D}\left(k_{2}-p_{2}\right)(2 \pi)^{D} \delta^{D}\left(q_{2}+p_{1}\right)\right] .
\end{aligned}
$$


Vamos ao cálculo das integrais. Inicialmente as integrais em $q_{1}$ e $q_{3}$;

$$
\begin{aligned}
\left\langle p_{1}\left|A_{2}\right| p_{2}\right\rangle & =-\frac{\lambda^{2}}{(2 \pi)^{D-1}} \int \frac{d \omega}{2 \pi i} \frac{1}{\omega+i \epsilon} \prod_{i=1}^{3} \int \frac{d^{D} k_{i}}{(2 \pi)^{D}} \int \frac{d^{D} q_{2}}{(2 \pi)^{D}} \\
& \times(2 \pi)^{D} \delta\left(k_{1}+k_{2}+k_{3}\right) \cos \left(\frac{k_{1} \wedge k_{2}}{2}\right)(2 \pi)^{D} \delta\left(-k_{1}+q_{2}-k_{3}\right) \\
& \times \cos \left(\frac{k_{1} \wedge q_{2}}{2}\right) \widetilde{S}_{\alpha \gamma}^{+}\left(k_{1}\right) \widetilde{\Delta}^{+}\left(k_{3}-\omega\right) \\
& \times\left[\bar{u}_{\alpha}^{\left(t_{1}\right)}\left(p_{1}\right) u_{\gamma}^{\left(t_{2}\right)}\left(p_{2}\right)(2 \pi)^{D} \delta^{D}\left(k_{2}+p_{1}\right)(2 \pi)^{D} \delta^{D}\left(q_{2}-p_{2}\right)\right. \\
& \left.-\bar{v}_{\alpha}^{\left(t_{2}\right)}\left(p_{2}\right) v_{\gamma}^{\left(t_{1}\right)}\left(p_{1}\right)(2 \pi)^{D} \delta^{D}\left(k_{2}-p_{2}\right)(2 \pi)^{D} \delta^{D}\left(q_{2}+p_{1}\right)\right] .
\end{aligned}
$$

A integral em $q_{2}$;

$$
\begin{aligned}
\left\langle p_{1}\left|A_{2}\right| p_{2}\right\rangle & =-\frac{\lambda^{2}}{(2 \pi)^{D-1}} \int \frac{d \omega}{2 \pi i} \frac{1}{\omega+i \epsilon} \prod_{i=1}^{3} \int \frac{d^{D} k_{i}}{(2 \pi)^{D}} \\
& \times(2 \pi)^{D} \delta\left(k_{1}+k_{2}+k_{3}\right) \cos ^{2}\left(\frac{k_{1} \wedge k_{2}}{2}\right) \widetilde{S}_{\alpha \gamma}^{+}\left(k_{1}\right) \widetilde{\Delta}^{+}\left(k_{3}-\omega\right) \\
& \times\left[\bar{u}_{\alpha}^{\left(t_{1}\right)}\left(p_{1}\right) u_{\gamma}^{\left(t_{2}\right)}\left(p_{2}\right)(2 \pi)^{D} \delta^{D}\left(k_{2}+p_{1}\right)(2 \pi)^{D} \delta^{D}\left(k_{1}+k_{3}-p_{2}\right)\right. \\
& \left.-\bar{v}_{\alpha}^{\left(t_{2}\right)}\left(p_{2}\right) v_{\gamma}^{\left(t_{1}\right)}\left(p_{1}\right)(2 \pi)^{D} \delta^{D}\left(k_{2}-p_{2}\right)(2 \pi)^{D} \delta^{D}\left(k_{1}+k_{3}+p_{1}\right)\right] .
\end{aligned}
$$

Integral em $k_{3}$;

$$
\begin{aligned}
\left\langle p_{1}\left|A_{2}\right| p_{2}\right\rangle & =-\frac{\lambda^{2}}{(2 \pi)^{D-1}} \int \frac{d \omega}{2 \pi i} \frac{1}{\omega+i \epsilon} \int \frac{d^{D} k_{1}}{(2 \pi)^{D}} \frac{d^{D} k_{2}}{(2 \pi)^{D}} \\
& \times \cos ^{2}\left(\frac{k_{1} \wedge k_{2}}{2}\right) \widetilde{S}_{\alpha \gamma}^{+}\left(k_{1}\right) \widetilde{\Delta}^{+}\left(-k_{1}-k_{2}-\omega\right) \\
& \times\left[\bar{u}_{\alpha}^{\left(t_{1}\right)}\left(p_{1}\right) u_{\gamma}^{\left(t_{2}\right)}\left(p_{2}\right)(2 \pi)^{D} \delta^{D}\left(k_{2}+p_{1}\right)(2 \pi)^{D} \delta^{D}\left(k_{2}+p_{2}\right)\right. \\
& \left.-\bar{v}_{\alpha}^{\left(t_{2}\right)}\left(p_{2}\right) v_{\gamma}^{\left(t_{1}\right)}\left(p_{1}\right)(2 \pi)^{D} \delta^{D}\left(k_{2}-p_{2}\right)(2 \pi)^{D} \delta^{D}\left(-k_{2}+p_{1}\right)\right] .
\end{aligned}
$$

Por fim, a integral em $k_{2}$; 


$$
\begin{aligned}
\left\langle p_{1}\left|A_{2}\right| p_{2}\right\rangle & =-(2 \pi)^{D} \delta^{D}\left(p_{2}-p_{1}\right) \frac{\lambda^{2}}{(2 \pi)^{D-1}} \int \frac{d \omega}{2 \pi i} \frac{1}{\omega+i \epsilon} \int \frac{d^{D} k_{1}}{(2 \pi)^{D}} \\
& \times\left[\bar{u}_{\alpha}^{\left(t_{1}\right)}\left(p_{1}\right) \widetilde{S}_{\alpha \gamma}^{+}\left(k_{1}\right) u_{\gamma}^{\left(t_{2}\right)}\left(p_{1}\right) \widetilde{\Delta}^{+}\left(-k_{1}+p_{1}-\omega\right) \cos ^{2}\left(\frac{k_{1} \wedge p_{1}}{2}\right)\right. \\
& \left.-\bar{v}_{\alpha}^{\left(t_{2}\right)}\left(p_{2}\right) \widetilde{S}_{\alpha \gamma}^{+}\left(k_{1}\right) v_{\gamma}^{\left(t_{1}\right)}\left(p_{2}\right) \widetilde{\Delta}^{+}\left(-k_{1}-p_{2}-\omega\right) \cos ^{2}\left(\frac{k_{1} \wedge p_{2}}{2}\right)\right] .
\end{aligned}
$$

Como no caso do modelo $\phi^{3}$, o lado esquerdo da condição de unitariedade (5.2) é essencialmente a parte real de $\left\langle p_{1}\left|A_{2}\right| p_{2}\right\rangle$. De acordo com a identidade (4.30), temos

$$
\begin{aligned}
\operatorname{Re}\left\langle p_{1}\left|A_{2}\right| p_{2}\right\rangle & =(2 \pi)^{D} \delta^{D}\left(p_{2}-p_{1}\right) \frac{\lambda^{2}}{(2 \pi)^{D-1}} \frac{1}{2} \int \frac{d^{D} k_{1}}{(2 \pi)^{D}} \\
& \times\left[\bar{u}_{\alpha}^{\left(t_{1}\right)}\left(p_{1}\right) \widetilde{S}_{\alpha \gamma}^{+}\left(k_{1}\right) u_{\gamma}^{\left(t_{2}\right)}\left(p_{1}\right) \widetilde{\Delta}^{+}\left(-k_{1}+p_{1}\right) \cos ^{2}\left(\frac{k_{1} \wedge p_{1}}{2}\right)\right. \\
& \left.-\bar{v}_{\alpha}^{\left(t_{2}\right)}\left(p_{2}\right) \widetilde{S}_{\alpha \gamma}^{+}\left(k_{1}\right) v_{\gamma}^{\left(t_{1}\right)}\left(p_{2}\right) \widetilde{\Delta}^{+}\left(-k_{1}-p_{2}\right) \cos ^{2}\left(\frac{k_{1} \wedge p_{2}}{2}\right)\right] .
\end{aligned}
$$

O fato que os produtos matriciais $\bar{u}_{\alpha}^{\left(t_{1}\right)}\left(p_{1}\right) \widetilde{S}_{\alpha \gamma}^{+}\left(k_{1}\right) u_{\gamma}^{\left(t_{2}\right)}\left(p_{1}\right)$ e $\bar{v}_{\alpha}^{\left(t_{2}\right)}\left(p_{2}\right) \widetilde{S}_{\alpha \gamma}^{+}\left(k_{1}\right) v_{\gamma}^{\left(t_{1}\right)}\left(p_{2}\right)$ são reais é provado no apêndice $E$.

Partimos agora para o cálculo do lado direito de (5.2). As etapas desse cálculo são as mesmas que as do caso anterior. Usando o fato que $A_{1}^{\dagger}=A_{1}$ (esse resultado é provado no apêndice $F$ ), temos

$$
\begin{aligned}
\left\langle p_{1}\left|A_{1}^{\dagger} A_{1}\right| p_{2}\right\rangle & =\frac{\lambda^{2}}{4} \int d^{D} x d^{D} y\left\langle p_{1}\left|\mathcal{F}_{x y}\left[\bar{\psi}_{\alpha}(x) \psi_{\alpha}(x) \phi(x) \bar{\psi}_{\gamma}(y) \psi_{\gamma}(y) \phi(y)\right]\right| p_{2}\right\rangle \\
& =\frac{\lambda^{2}}{4} \int d^{D} x d^{D} y\left\langle p_{1}\left|\left(\bar{\psi}_{\alpha}(x) * \psi_{\alpha}(x) * \phi(x)\right)\left(\bar{\psi}_{\gamma}(y) * \psi_{\gamma}(y) * \phi(y)\right)\right| p_{2}\right\rangle .
\end{aligned}
$$

De acordo com (5.6), podemos escrever, 


$$
\begin{aligned}
\left\langle p_{1}\left|A_{1}^{\dagger} A_{1}\right| p_{2}\right\rangle & =\frac{\lambda^{2}}{4} \prod_{i=1}^{3} \int \frac{d^{D} k_{i}}{(2 \pi)^{D}} \prod_{j=1}^{3} \int \frac{d^{D} q_{j}}{(2 \pi)^{D}}(2 \pi)^{D} \delta^{D}\left(k_{1}+k_{2}+k_{3}\right) \\
& \times \cos \left(\frac{k_{1} \wedge k_{2}}{2}\right)(2 \pi)^{D} \delta^{D}\left(q_{1}+q_{2}+q_{3}\right) \cos \left(\frac{q_{1} \wedge q_{2}}{2}\right) \\
& \times\left\langle p_{1}\left|\widetilde{\bar{\psi}}_{\alpha}\left(k_{1}\right) \widetilde{\psi}_{\alpha}\left(k_{2}\right) \widetilde{\phi}\left(k_{3}\right) \widetilde{\bar{\psi}}_{\gamma}\left(q_{1}\right) \widetilde{\psi}_{\gamma}\left(q_{2}\right) \widetilde{\phi}\left(q_{3}\right)\right| p_{2}\right\rangle .
\end{aligned}
$$

Aplicando o teorema de Wick e selecionando apenas os termos que contribuem para o diagrama (5.1), chegamos à

$$
\begin{aligned}
\left\langle p_{1}\left|A_{1}^{\dagger} A_{1}\right| p_{2}\right\rangle & =\frac{\lambda^{2}}{4} \prod_{i=1}^{3} \int \frac{d^{D} k_{i}}{(2 \pi)^{D}} \prod_{j=1}^{3} \int \frac{d^{D} q_{j}}{(2 \pi)^{D}}(2 \pi)^{D} \delta^{D}\left(k_{1}+k_{2}+k_{3}\right) \\
& \times \cos \left(\frac{k_{1} \wedge k_{2}}{2}\right)(2 \pi)^{D} \delta^{D}\left(q_{1}+q_{2}+q_{3}\right) \cos \left(\frac{q_{1} \wedge q_{2}}{2}\right) \\
& \times 4(2 \pi)^{D} \delta^{D}\left(k_{1}+q_{1}\right) \widetilde{S}_{\alpha \gamma}^{+}\left(k_{1}\right)(2 \pi)^{D} \delta^{D}\left(k_{3}+q_{3}\right) \widetilde{\Delta}^{+}\left(k_{3}\right) \\
& \times\left\langle p_{1}\left|: \widetilde{\bar{\psi}}_{\alpha}\left(k_{2}\right) \widetilde{\psi}_{\gamma}\left(q_{2}\right):\right| p_{2}\right\rangle .
\end{aligned}
$$

Esse elemento de matriz é justamente aquele em (5.26). Então,

$$
\begin{aligned}
\left\langle p_{1}\left|A_{1}^{\dagger} A_{1}\right| p_{2}\right\rangle & =\frac{\lambda^{2}}{(2 \pi)^{D-1}} \prod_{i=1}^{3} \int \frac{d^{D} k_{i}}{(2 \pi)^{D}} \prod_{j=1}^{3} \int \frac{d^{D} q_{j}}{(2 \pi)^{D}}(2 \pi)^{D} \delta^{D}\left(k_{1}+k_{2}+k_{3}\right) \\
& \times \cos \left(\frac{k_{1} \wedge k_{2}}{2}\right)(2 \pi)^{D} \delta^{D}\left(q_{1}+q_{2}+q_{3}\right) \cos \left(\frac{q_{1} \wedge q_{2}}{2}\right) \\
& \times(2 \pi)^{D} \delta^{D}\left(k_{1}+q_{1}\right) \widetilde{S}_{\alpha \gamma}^{+}\left(k_{1}\right)(2 \pi)^{D} \delta^{D}\left(k_{3}+q_{3}\right) \widetilde{\Delta}^{+}\left(k_{3}\right) \\
& \times\left[\bar{u}_{\alpha}^{\left(t_{1}\right)}\left(p_{1}\right) u_{\gamma}^{\left(t_{2}\right)}\left(p_{2}\right)(2 \pi)^{D} \delta^{D}\left(k_{2}+p_{1}\right)(2 \pi)^{D} \delta^{D}\left(q_{2}-p_{2}\right)\right. \\
& \left.-\bar{v}_{\alpha}^{\left(t_{2}\right)}\left(p_{2}\right) v_{\gamma}^{\left(t_{1}\right)}\left(p_{1}\right)(2 \pi)^{D} \delta^{D}\left(k_{2}-p_{2}\right)(2 \pi)^{D} \delta^{D}\left(q_{2}+p_{1}\right)\right] .
\end{aligned}
$$

Basta calcular as integrais. Primeiramente, as integrais em $q_{1}$ e $q_{3}$; 
Modelo de Yukawa

$$
\begin{aligned}
\left\langle p_{1}\left|A_{1}^{\dagger} A_{1}\right| p_{2}\right\rangle & =\frac{\lambda^{2}}{(2 \pi)^{D-1}} \prod_{i=1}^{3} \int \frac{d^{D} k_{i}}{(2 \pi)^{D}} \int \frac{d^{D} q_{2}}{(2 \pi)^{D}}(2 \pi)^{D} \delta^{D}\left(k_{1}+k_{2}+k_{3}\right) \\
& \times \cos \left(\frac{k_{1} \wedge k_{2}}{2}\right)(2 \pi)^{D} \delta^{D}\left(-k_{1}+q_{2}-k_{3}\right) \cos \left(\frac{k_{1} \wedge q_{2}}{2}\right) \\
& \times \widetilde{S}_{\alpha \gamma}^{+}\left(k_{1}\right) \widetilde{\Delta}^{+}\left(k_{3}\right) \\
& \times\left[\bar{u}_{\alpha}^{\left(t_{1}\right)}\left(p_{1}\right) u_{\gamma}^{\left(t_{2}\right)}\left(p_{2}\right)(2 \pi)^{D} \delta^{D}\left(k_{2}+p_{1}\right)(2 \pi)^{D} \delta^{D}\left(q_{2}-p_{2}\right)\right. \\
& \left.-\bar{v}_{\alpha}^{\left(t_{2}\right)}\left(p_{2}\right) v_{\gamma}^{\left(t_{1}\right)}\left(p_{1}\right)(2 \pi)^{D} \delta^{D}\left(k_{2}-p_{2}\right)(2 \pi)^{D} \delta^{D}\left(q_{2}+p_{1}\right)\right] .
\end{aligned}
$$

Integral em $q_{2}$;

$$
\begin{aligned}
\left\langle p_{1}\left|A_{1}^{\dagger} A_{1}\right| p_{2}\right\rangle & =\frac{\lambda^{2}}{(2 \pi)^{D-1}} \prod_{i=1}^{3} \int \frac{d^{D} k_{i}}{(2 \pi)^{D}}(2 \pi)^{D} \delta^{D}\left(k_{1}+k_{2}+k_{3}\right) \\
& \times \cos ^{2}\left(\frac{k_{1} \wedge k_{2}}{2}\right) \widetilde{S}_{\alpha \gamma}^{+}\left(k_{1}\right) \widetilde{\Delta}^{+}\left(k_{3}\right) \\
& \times\left[\bar{u}_{\alpha}^{\left(t_{1}\right)}\left(p_{1}\right) u_{\gamma}^{\left(t_{2}\right)}\left(p_{2}\right)(2 \pi)^{D} \delta^{D}\left(k_{2}+p_{1}\right)(2 \pi)^{D} \delta^{D}\left(k_{1}+k_{3}-p_{2}\right)\right. \\
& \left.-\bar{v}_{\alpha}^{\left(t_{2}\right)}\left(p_{2}\right) v_{\gamma}^{\left(t_{1}\right)}\left(p_{1}\right)(2 \pi)^{D} \delta^{D}\left(k_{2}-p_{2}\right)(2 \pi)^{D} \delta^{D}\left(k_{1}+k_{3}+p_{1}\right)\right] .
\end{aligned}
$$

Integral em $k_{3}$;

$$
\begin{aligned}
\left\langle p_{1}\left|A_{1}^{\dagger} A_{1}\right| p_{2}\right\rangle & =\frac{\lambda^{2}}{(2 \pi)^{D-1}} \int \frac{d^{D} k_{1}}{(2 \pi)^{D}} \int \frac{d^{D} k_{2}}{(2 \pi)^{D}} \cos ^{2}\left(\frac{k_{1} \wedge k_{2}}{2}\right) \widetilde{S}_{\alpha \gamma}^{+}\left(k_{1}\right) \widetilde{\Delta}^{+}\left(-k_{1}-k_{2}\right) \\
& \times\left[\bar{u}_{\alpha}^{\left(t_{1}\right)}\left(p_{1}\right) u_{\gamma}^{\left(t_{2}\right)}\left(p_{2}\right)(2 \pi)^{D} \delta^{D}\left(k_{2}+p_{1}\right)(2 \pi)^{D} \delta^{D}\left(k_{2}+p_{2}\right)\right. \\
& \left.-\bar{v}_{\alpha}^{\left(t_{2}\right)}\left(p_{2}\right) v_{\gamma}^{\left(t_{1}\right)}\left(p_{1}\right)(2 \pi)^{D} \delta^{D}\left(k_{2}-p_{2}\right)(2 \pi)^{D} \delta^{D}\left(-k_{2}+p_{1}\right)\right] .
\end{aligned}
$$

Por último, a integral em $k_{2}$; 


$$
\begin{aligned}
\left\langle p_{1}\left|A_{1}^{\dagger} A_{1}\right| p_{2}\right\rangle & =(2 \pi)^{D} \delta^{D}\left(p_{2}-p_{1}\right) \frac{\lambda^{2}}{(2 \pi)^{D-1}} \int \frac{d^{D} k_{1}}{(2 \pi)^{D}} \\
& \times\left[\bar{u}_{\alpha}^{\left(t_{1}\right)}\left(p_{1}\right) \widetilde{S}_{\alpha \gamma}^{+}\left(k_{1}\right) u_{\gamma}^{\left(t_{2}\right)}\left(p_{1}\right) \widetilde{\Delta}^{+}\left(-k_{1}+p_{1}\right) \cos ^{2}\left(\frac{k_{1} \wedge p_{1}}{2}\right)\right. \\
& \left.-\bar{v}_{\alpha}^{\left(t_{2}\right)}\left(p_{2}\right) \widetilde{S}_{\alpha \gamma}^{+}\left(k_{1}\right) v_{\gamma}^{\left(t_{1}\right)}\left(p_{2}\right) \widetilde{\Delta}^{+}\left(-k_{1}-p_{2}\right) \cos ^{2}\left(\frac{k_{1} \wedge p_{2}}{2}\right)\right] .
\end{aligned}
$$

Comparando esse resultado com (5.32), verificamos que a relação de unitariedade

$$
2 \operatorname{Re}\left\langle p_{1}\left|A_{2}\right| p_{2}\right\rangle=\left\langle p_{1}\left|A_{1}^{\dagger} A_{1}\right| p_{2}\right\rangle
$$

é satisfeita para o diagrama considerado. Como dissemos no início desta seção, precisamos ainda investigar outro diagrama que contribui para a relação (5.2) quando os estados $\left|p_{1}\right\rangle$ e $\left|p_{2}\right\rangle$ representam partículas bosônicas. Isto será feito a seguir.

\subsection{Diagrama com pernas externas bosônicas}

Prosseguindo nossa análise da unitariedade a um laço, vamos estudar o diagrama mostrado na figura (5.2).

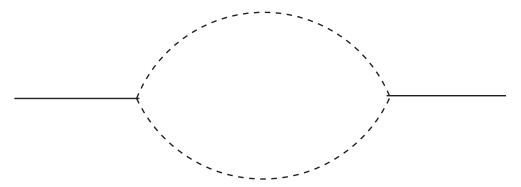

Figura 5.2: Diagrama de um laço com pernas externas bosônicas.

As etapas aqui são essencialmente as mesmas que aquelas feitas no caso anterior. A diferença vem dos tipos de contrações que serão consideradas na aplicação do teorema de Wick pois, os termos que contribuem para o diagrama são aqueles em que todos os campos fermiônicos estão contraídos. Dessa maneira, nosso ponto de partida é a amplitude

$$
\left\langle p_{1}\left|A_{2}\right| p_{2}\right\rangle=\left(\frac{\lambda}{2}\right)^{2} \int d^{D} x d^{D} y\left\langle p_{1}\left|\mathcal{F}_{x y}\left[\theta\left(x^{0}-y^{0}\right) \bar{\psi}_{\alpha}(x) \psi_{\alpha}(x) \phi(x) \bar{\psi}_{\gamma}(y) \psi_{\gamma}(y) \phi(y)\right]\right| p_{2}\right\rangle
$$

Vale relembrar que, apesar dos estados $\left|p_{1}\right\rangle$ e $\left|p_{2}\right\rangle$ estarem representados da mesma forma que no caso anterior há uma diferença fundamental entre eles: aqui representam partículas 
bosônicas, em contraste com o caso anterior em que referiam-se a férmions. Usando a representação integral para a função $\theta$, fazemos a escolha para realização minimal tal que

$$
\begin{aligned}
\left\langle p_{1}\left|A_{2}\right| p_{2}\right\rangle & =-\frac{\lambda^{2}}{4} \int d^{D} x d^{D} y \int \frac{d \omega}{2 \pi i} \frac{1}{\omega+i \epsilon} \\
& \times\left\langle p_{1}\left|\left(\bar{\psi}_{\alpha}(x) *\left(e^{-i \omega x} \psi_{\alpha}(x)\right) * \phi(x)\right)\left(\left(e^{i \omega y} \bar{\psi}_{\gamma}(y)\right) * \psi_{\gamma}(y) * \phi(y)\right)\right| p_{2}\right\rangle .
\end{aligned}
$$

Deve ser ressaltado que a escolha para realização minimal deve ser entre os campos fermiônicos, pois eles estarão contraídos nos termos que contribuem para o diagrama (5.2). No cálculo do diagrama anterior nos valemos da simetria existente entre os férmions de Majorana para escrever o produto Moyal na forma (5.6). Notemos que aqui essa simetria (referindo-se a aparência do fator coseno) não está completamente presente. Para ver isso, consideremos o produto Moyal entre os campos com argumento $x$ presente na expressão acima;

$$
\begin{aligned}
& \int d^{D} x \bar{\psi}_{\alpha}(x) *\left(e^{-i \omega x} \psi_{\alpha}(x)\right) * \phi(x) \\
= & \int \frac{d^{D} k_{1}}{(2 \pi)^{D}} \frac{d^{D} k_{2}}{(2 \pi)^{D}} \frac{d^{D} k_{3}}{(2 \pi)^{D}}(2 \pi)^{D} \delta^{D}\left(k_{1}+k_{2}+k_{3}\right) e^{-\frac{i}{2} k_{1} \wedge k_{2}} \widetilde{\bar{\psi}}_{\alpha}\left(k_{1}\right) \widetilde{\psi}_{\alpha}\left(k_{2}-\omega\right) \widetilde{\phi}\left(k_{3}\right) \\
= & \int \frac{d^{D} k_{1}}{(2 \pi)^{D}} \frac{d^{D} k_{2}}{(2 \pi)^{D}} \frac{d^{D} k_{3}}{(2 \pi)^{D}}(2 \pi)^{D} \delta^{D}\left(k_{1}+k_{2}+k_{3}\right) \\
\times & e^{\frac{i}{4} \omega \wedge\left(k_{1}+k_{2}\right)} \cos \left(\frac{1}{2} k_{1} \wedge k_{2}+\frac{1}{4} \omega \wedge\left(k_{1}+k_{2}\right)\right) \widetilde{\bar{\psi}}_{\alpha}\left(k_{1}\right) \widetilde{\psi}_{\alpha}\left(k_{2}-\omega\right) \widetilde{\phi}\left(k_{3}\right) .
\end{aligned}
$$

Esse resultado é demonstrado no apêndice G. Deve ser notado que, diferentemente de (5.6), na relação acima além do coseno há uma fase dependente de $\omega$.

De maneira análoga, temos o seguinte resultado para o produto Moyal entre os campos com argumento $y$ em (5.43):

$$
\begin{aligned}
& \int d^{D} y\left(e^{i \omega y} \bar{\psi}_{\gamma}(y)\right) * \psi_{\gamma}(y) * \phi(y) \\
= & \int \frac{d^{D} q_{1}}{(2 \pi)^{D}} \frac{d^{D} q_{2}}{(2 \pi)^{D}} \frac{d^{D} q_{3}}{(2 \pi)^{D}}(2 \pi)^{D} \delta^{D}\left(q_{1}+q_{2}+q_{3}\right) \\
\times & e^{\frac{i}{4} \omega \wedge\left(q_{1}+q_{2}\right)} \cos \left(\frac{1}{2} q_{1} \wedge q_{2}+\frac{1}{4} \omega \wedge\left(q_{1}+q_{2}\right)\right) \widetilde{\bar{\psi}}_{\gamma}\left(q_{1}+\omega\right) \widetilde{\psi}_{\gamma}\left(q_{2}\right) \widetilde{\phi}\left(q_{3}\right) .
\end{aligned}
$$

Substituindo (5.44) e (5.45) em (5.43) ficamos com 


$$
\begin{aligned}
\left\langle p_{1}\left|A_{2}\right| p_{2}\right\rangle & =-\frac{\lambda^{2}}{4} \int \frac{d \omega}{2 \pi i} \frac{1}{\omega+i \epsilon} \prod_{i=1}^{3} \int \frac{d^{D} k_{i}}{(2 \pi)^{D}}(2 \pi)^{D} \delta^{D}\left(k_{1}+k_{2}+k_{3}\right) e^{\frac{i}{4} \omega \wedge\left(k_{1}+k_{2}\right)} \\
& \times \cos \left(\frac{1}{2} k_{1} \wedge k_{2}+\frac{1}{4} \omega \wedge\left(k_{1}+k_{2}\right)\right) \prod_{j=1}^{3} \int \frac{d^{D} q_{i}}{(2 \pi)^{D}}(2 \pi)^{D} \delta^{D}\left(q_{1}+q_{2}+q_{3}\right) \\
& \times e^{\frac{i}{4} \omega \wedge\left(q_{1}+q_{2}\right)} \cos \left(\frac{1}{2} q_{1} \wedge q_{2}+\frac{1}{4} \omega \wedge\left(q_{1}+q_{2}\right)\right) \\
& \times\left\langle p_{1}\left|\widetilde{\bar{\psi}}_{\alpha}\left(k_{1}\right) \widetilde{\psi}_{\alpha}\left(k_{2}-\omega\right) \widetilde{\phi}\left(k_{3}\right) \widetilde{\bar{\psi}}_{\gamma}\left(q_{1}+\omega\right) \widetilde{\psi}_{\gamma}\left(q_{2}\right) \widetilde{\phi}\left(q_{3}\right)\right| p_{2}\right\rangle .
\end{aligned}
$$

Agora, vamos aplicar o teorema de Wick selecionando apenas os termos do elemento de matriz na última linha que contribuem para o diagrama (5.2). Desse modo,

$$
\begin{aligned}
& \left\langle p_{1}\left|\widetilde{\bar{\psi}}_{\alpha}\left(k_{1}\right) \widetilde{\psi}_{\alpha}\left(k_{2}-\omega\right) \widetilde{\phi}\left(k_{3}\right) \widetilde{\bar{\psi}}_{\gamma}\left(q_{1}+\omega\right) \widetilde{\psi}_{\gamma}\left(q_{2}\right) \widetilde{\phi}\left(q_{3}\right)\right| p_{2}\right\rangle \\
= & -\widetilde{\bar{\psi}}_{\alpha}^{c_{1}}\left(k_{1}\right) \widetilde{\bar{\psi}}_{\gamma}^{c_{1}}\left(q_{1}+\omega\right) \widetilde{\psi}_{\alpha}^{c_{2}}\left(k_{2}-\omega\right) \widetilde{\psi}_{\gamma}^{c_{2}}\left(q_{2}\right)\left\langle p_{1}\left|: \widetilde{\phi}\left(k_{3}\right) \widetilde{\phi}\left(q_{3}\right):\right| p_{2}\right\rangle \\
+ & \widetilde{\bar{\psi}}_{\alpha}^{c_{1}}\left(k_{1}\right) \widetilde{\psi}_{\gamma}^{c_{1}}\left(q_{2}\right) \widetilde{\psi}_{\alpha}^{c_{2}}\left(k_{2}-\omega\right) \widetilde{\bar{\psi}}_{\gamma}^{c_{2}}\left(q_{1}+\omega\right)\left\langle p_{1}\left|: \widetilde{\phi}\left(k_{3}\right) \widetilde{\phi}\left(q_{3}\right):\right| p_{2}\right\rangle .
\end{aligned}
$$

Nesse ponto devemos fazer algumas observações. Diferentemente do que foi feito nos casos anteriores, na expressão acima consideramos um termo vindo do teorema de Wick em que os campos cujas exponenciais da função $\theta$ foram incorporadas em campos que não estão contraídos entre si, a saber, o primeiro termo. Mas de acordo com a prescrição da realização minimal a função $\theta$ deve ser incorporada em campos que estão contraídos. Problema semelhante ocorreu no estudo do modelo $\phi^{3}$ no quarto capítulo. Lá, devido ao teorema de Wick, o número total de termos que contribuem para o diagrama de um laço é 36. Porém, para uma escolha particular da realização minimal, haviam apenas quatro termos. Os demais (32) termos eram gerados considerando-se as outras possibilidades para realização minimal que no final totalizavam $9 \times 4$ termos. Em suma, há a necessidade de conciliar o teorema de Wick com a prescrição minimal. De maneira completamente equivalente, poderíamos ter considerado uma escolha particular para a prescrição e então fazer todas as contrações (36) mesmo que ocorram contrações que não coincidam com a escolha minimal. Em seguida, basta fazer mudanças de variáveis de modo que todos os termos tenham a forma exigida pela prescrição. Além disso, veríamos que todos eles são iguais.

Para tratar (5.47), adotaremos o procedimento descrito acima, ou seja, por meio de uma mudança de variável mostraremos que os dois termos em (5.47) ficam na forma desejada, além deles darem a mesma contribuição. De fato, uma possível mudança é $q_{2} \leftrightarrow q_{1}+\omega$ no primeiro termo. Antes de analisar propriamente esse termo, lembramos que há outros fatores dependentes de $q_{1}$ e $q_{2}$ multiplicando-o. A saber, a delta de conservação que claramente não é alterada; a fase e o fator de coseno que, com a mudança ficam 


$$
\begin{aligned}
& e^{\frac{i}{4} \omega \wedge\left(q_{2}-\omega+q_{1}+\omega\right)} \cos \left(\frac{1}{2}\left(q_{2}-\omega\right) \wedge\left(q_{1}+\omega\right)+\frac{1}{4} \omega \wedge\left(q_{2}-\omega+q_{1}+\omega\right)\right) \\
= & e^{\frac{i}{4} \omega \wedge\left(q_{1}+q_{2}\right)} \cos \left(\frac{1}{2} q_{1} \wedge q_{2}+\frac{1}{4} \omega \wedge\left(q_{1}+q_{2}\right)\right),
\end{aligned}
$$

ou seja, também não são alterados, como deveria ser! Agora, analisemos os termos de (5.47) após a mudança:

$$
\begin{aligned}
& -\widetilde{\bar{\psi}}_{\alpha}^{c_{1}}\left(k_{1}\right) \widetilde{\bar{\psi}}_{\gamma}^{c_{1}}\left(q_{2}\right) \widetilde{\psi}_{\alpha}^{c_{2}}\left(k_{2}-\omega\right) \widetilde{\psi}_{\gamma}^{c_{2}}\left(q_{1}+\omega\right)\left\langle p_{1}\left|: \widetilde{\phi}\left(k_{3}\right) \widetilde{\phi}\left(q_{3}\right):\right| p_{2}\right\rangle \\
+ & \widetilde{\bar{\psi}}_{\alpha}^{c_{1}}\left(k_{1}\right) \widetilde{\psi}_{\gamma}^{c_{1}}\left(q_{2}\right) \widetilde{\psi}_{\alpha}^{c_{2}}\left(k_{2}-\omega\right) \widetilde{\bar{\psi}}_{\gamma}^{c_{2}}\left(q_{1}+\omega\right)\left\langle p_{1}\left|: \widetilde{\phi}\left(k_{3}\right) \widetilde{\phi}\left(q_{3}\right):\right| p_{2}\right\rangle \\
= & -C_{\beta \alpha}^{-1} C_{\lambda \gamma} \widetilde{\psi}_{\beta}^{c_{1}}\left(k_{1}\right) \widetilde{\bar{\psi}}_{\gamma}^{c_{1}}\left(q_{2}\right) \widetilde{\psi}_{\alpha}^{c_{2}}\left(k_{2}-\omega\right) \widetilde{\bar{\psi}}_{\lambda}^{c_{2}}\left(q_{1}+\omega\right)\left\langle p_{1}\left|: \widetilde{\phi}\left(k_{3}\right) \widetilde{\phi}\left(q_{3}\right):\right| p_{2}\right\rangle \\
+ & C_{\beta \alpha}^{-1} C_{\lambda \gamma} \widetilde{\psi}_{\beta}^{c_{1}}\left(k_{1}\right) \widetilde{\bar{\psi}}_{\lambda}^{c_{1}}\left(q_{2}\right) \widetilde{\psi}_{\alpha}^{c_{2}}\left(k_{2}-\omega\right) \widetilde{\bar{\psi}}_{\gamma}^{c_{2}}\left(q_{1}+\omega\right)\left\langle p_{1}\left|: \widetilde{\phi}\left(k_{3}\right) \widetilde{\phi}\left(q_{3}\right):\right| p_{2}\right\rangle \\
= & 2 C_{\beta \alpha}^{-1} C_{\lambda \gamma} \widetilde{\psi}_{\beta}^{c_{1}}\left(k_{1}\right) \widetilde{\bar{\psi}}_{\lambda}^{c_{1}}\left(q_{2}\right) \widetilde{\psi}_{\alpha}^{c_{2}}\left(k_{2}-\omega\right) \widetilde{\bar{\psi}}_{\gamma}^{c_{2}}\left(q_{1}+\omega\right)\left\langle p_{1}\left|: \widetilde{\phi}\left(k_{3}\right) \widetilde{\phi}\left(q_{3}\right):\right| p_{2}\right\rangle \\
= & 2 C_{\beta \alpha}^{-1} C_{\lambda \gamma}(2 \pi)^{D} \delta^{D}\left(k_{1}+q_{2}\right) \widetilde{S}_{\beta \lambda}^{+}\left(k_{1}\right)(2 \pi)^{D} \delta^{D}\left(k_{2}+q_{1}\right) \widetilde{S}_{\alpha \gamma}^{+}\left(k_{2}-\omega\right) \\
\times & \left\langle p_{1}\left|: \widetilde{\phi}\left(k_{3}\right) \widetilde{\phi}\left(q_{3}\right):\right| p_{2}\right\rangle,
\end{aligned}
$$

sendo que para obter a penúltima igualdade renomeamos alguns índices e usamos as propriedades da matriz de conjugação de carga. Na última igualdade empregamos a contração (5.15). O elemento de matriz com os campos bosônicos é dado em (4.23). Levando todos esses resultados em (5.46), obtemos,

$$
\begin{aligned}
\left\langle p_{1}\left|A_{2}\right| p_{2}\right\rangle & =-\frac{\lambda^{2}}{4} \int \frac{d \omega}{2 \pi i} \frac{1}{\omega+i \epsilon} \prod_{i=1}^{3} \int \frac{d^{D} k_{i}}{(2 \pi)^{D}}(2 \pi)^{D} \delta^{D}\left(k_{1}+k_{2}+k_{3}\right) e^{\frac{i}{4} \omega \wedge\left(k_{1}+k_{2}\right)} \\
& \times \cos \left(\frac{1}{2} k_{1} \wedge k_{2}+\frac{1}{4} \omega \wedge\left(k_{1}+k_{2}\right)\right) \prod_{j=1}^{3} \int \frac{d^{D} q_{i}}{(2 \pi)^{D}}(2 \pi)^{D} \delta^{D}\left(q_{1}+q_{2}+q_{3}\right) \\
& \times e^{\frac{i}{4} \omega \wedge\left(q_{1}+q_{2}\right)} \cos \left(\frac{1}{2} q_{1} \wedge q_{2}+\frac{1}{4} \omega \wedge\left(q_{1}+q_{2}\right)\right) \\
& \times 2 C_{\beta \alpha}^{-1} C_{\lambda \gamma}(2 \pi)^{D} \delta^{D}\left(k_{1}+q_{2}\right) \widetilde{S}_{\beta \lambda}^{+}\left(k_{1}\right)(2 \pi)^{D} \delta^{D}\left(k_{2}+q_{1}\right) \widetilde{S}_{\alpha \gamma}^{+}\left(k_{2}-\omega\right) \\
& \times \frac{1}{(2 \pi)^{D-1}}\left[(2 \pi)^{D} \delta^{D}\left(k_{3}-p_{2}\right)(2 \pi)^{D} \delta^{D}\left(q_{3}+p_{1}\right)\right. \\
& \left.+(2 \pi)^{D} \delta^{D}\left(k_{3}+p_{1}\right)(2 \pi)^{D} \delta^{D}\left(q_{3}-p_{2}\right)\right]
\end{aligned}
$$

Seguimos com o cálculo das integrais. Primeiramente, em $q_{1}$ e $q_{2}$; 


$$
\begin{aligned}
\left\langle p_{1}\left|A_{2}\right| p_{2}\right\rangle & =-\frac{\lambda^{2}}{2(2 \pi)^{D-1}} C_{\beta \alpha}^{-1} C_{\lambda \gamma} \int \frac{d \omega}{2 \pi i} \frac{1}{\omega+i \epsilon} \prod_{i=1}^{3} \int \frac{d^{D} k_{i}}{(2 \pi)^{D}}(2 \pi)^{D} \delta^{D}\left(k_{1}+k_{2}+k_{3}\right) \\
& \times \cos ^{2}\left(\frac{1}{2} k_{1} \wedge k_{2}+\frac{1}{4} \omega \wedge\left(k_{1}+k_{2}\right)\right) \int \frac{d^{D} q_{3}}{(2 \pi)^{D}}(2 \pi)^{D} \delta^{D}\left(-k_{1}-k_{2}+q_{3}\right) \\
& \times \widetilde{S}_{\beta \lambda}^{+}\left(k_{1}\right) \widetilde{S}_{\alpha \gamma}^{+}\left(k_{2}-\omega\right)\left[(2 \pi)^{D} \delta^{D}\left(k_{3}-p_{2}\right)(2 \pi)^{D} \delta^{D}\left(q_{3}+p_{1}\right)\right. \\
& \left.+(2 \pi)^{D} \delta^{D}\left(k_{3}+p_{1}\right)(2 \pi)^{D} \delta^{D}\left(q_{3}-p_{2}\right)\right] .
\end{aligned}
$$

Note que as fases se cancelam exatamente! A integral em $q_{3}$ dá

$$
\begin{aligned}
\left\langle p_{1}\left|A_{2}\right| p_{2}\right\rangle & =-\frac{\lambda^{2}}{2(2 \pi)^{D-1}} C_{\beta \alpha}^{-1} C_{\lambda \gamma} \int \frac{d \omega}{2 \pi i} \frac{1}{\omega+i \epsilon} \prod_{i=1}^{3} \int \frac{d^{D} k_{i}}{(2 \pi)^{D}}(2 \pi)^{D} \delta^{D}\left(k_{1}+k_{2}+k_{3}\right) \\
& \times \cos ^{2}\left(\frac{1}{2} k_{1} \wedge k_{2}+\frac{1}{4} \omega \wedge\left(k_{1}+k_{2}\right)\right) \\
& \times \widetilde{S}_{\beta \lambda}^{+}\left(k_{1}\right) \widetilde{S}_{\alpha \gamma}^{+}\left(k_{2}-\omega\right)\left[(2 \pi)^{D} \delta^{D}\left(k_{3}-p_{2}\right)(2 \pi)^{D} \delta^{D}\left(k_{1}+k_{2}+p_{1}\right)\right. \\
& \left.+(2 \pi)^{D} \delta^{D}\left(k_{3}+p_{1}\right)(2 \pi)^{D} \delta^{D}\left(k_{1}+k_{2}-p_{2}\right)\right]
\end{aligned}
$$

Integral em $k_{3}$;

$$
\begin{aligned}
\left\langle p_{1}\left|A_{2}\right| p_{2}\right\rangle & =-\frac{\lambda^{2}}{2(2 \pi)^{D-1}} C_{\beta \alpha}^{-1} C_{\lambda \gamma} \int \frac{d \omega}{2 \pi i} \frac{1}{\omega+i \epsilon} \int \frac{d^{D} k_{1}}{(2 \pi)^{D}} \int \frac{d^{D} k_{2}}{(2 \pi)^{D}} \\
& \times \cos ^{2}\left(\frac{1}{2} k_{1} \wedge k_{2}+\frac{1}{4} \omega \wedge\left(k_{1}+k_{2}\right)\right) \\
& \times \widetilde{S}_{\beta \lambda}^{+}\left(k_{1}\right) \widetilde{S}_{\alpha \gamma}^{+}\left(k_{2}-\omega\right)\left[(2 \pi)^{D} \delta^{D}\left(-k_{1}-k_{2}-p_{2}\right)(2 \pi)^{D} \delta^{D}\left(k_{1}+k_{2}+p_{1}\right)\right. \\
& \left.+(2 \pi)^{D} \delta^{D}\left(-k_{1}-k_{2}+p_{1}\right)(2 \pi)^{D} \delta^{D}\left(k_{1}+k_{2}-p_{2}\right)\right] .
\end{aligned}
$$

Por fim, a integral em $k_{2}$;

$$
\begin{aligned}
\left\langle p_{1}\left|A_{2}\right| p_{2}\right\rangle & =-(2 \pi)^{D} \delta^{D}\left(p_{2}-p_{1}\right) \frac{\lambda^{2}}{2(2 \pi)^{D-1}} C_{\beta \alpha}^{-1} C_{\lambda \gamma} \int \frac{d \omega}{2 \pi i} \frac{1}{\omega+i \epsilon} \int \frac{d^{D} k_{1}}{(2 \pi)^{D}} \\
& \times\left[\cos ^{2}\left(\frac{1}{2} k_{1} \wedge p_{2}+\frac{1}{4} \omega \wedge p_{2}\right) \widetilde{S}_{\beta \lambda}^{+}\left(k_{1}\right) \widetilde{S}_{\alpha \gamma}^{+}\left(-k_{1}-p_{2}-\omega\right)\right. \\
& \left.+\cos ^{2}\left(\frac{1}{2} k_{1} \wedge p_{1}+\frac{1}{4} \omega \wedge p_{1}\right) \widetilde{S}_{\beta \lambda}^{+}\left(k_{1}\right) \widetilde{S}_{\alpha \gamma}^{+}\left(-k_{1}+p_{1}-\omega\right)\right] .
\end{aligned}
$$


Separando a parte real de acordo com a identidade (4.30), chegamos ao seguinte resultado:

$$
\begin{aligned}
\operatorname{Re}\left\langle p_{1}\left|A_{2}\right| p_{2}\right\rangle & =(2 \pi)^{D} \delta^{D}\left(p_{2}-p_{1}\right) \frac{\lambda^{2}}{4(2 \pi)^{D-1}} C_{\beta \alpha}^{-1} C_{\lambda \gamma} \int \frac{d^{D} k_{1}}{(2 \pi)^{D}} \\
& \times\left[\cos ^{2}\left(\frac{1}{2} k_{1} \wedge p_{2}\right) \widetilde{S}_{\beta \lambda}^{+}\left(k_{1}\right) \widetilde{S}_{\alpha \gamma}^{+}\left(-k_{1}-p_{2}\right)\right. \\
& \left.+\cos ^{2}\left(\frac{1}{2} k_{1} \wedge p_{1}\right) \widetilde{S}_{\beta \lambda}^{+}\left(k_{1}\right) \widetilde{S}_{\alpha \gamma}^{+}\left(-k_{1}+p_{1}\right)\right] .
\end{aligned}
$$

Não é difícil verificar que um termo do tipo $C_{\beta \alpha}^{-1} C_{\lambda \gamma} \widetilde{S}_{\beta \lambda}^{+} \widetilde{S}_{\alpha \gamma}^{+}$é real.

O próximo passo é o cálculo do lado direito da condição de unitariedade (5.2). O procedimento é exatamente o mesmo que o anterior, porém sem a presença da função $\theta$ (e sem a realização minimal) o que o torna mais simples. Na sequência, apresentamos apenas o resultado sendo que os detalhes do cálculo são deixados para o apêndice H. Assim,

$$
\begin{aligned}
\left\langle p_{1}\left|A_{1}^{\dagger} A_{1}\right| p_{2}\right\rangle & =(2 \pi)^{D} \delta^{D}\left(p_{2}-p_{1}\right) \frac{\lambda^{2}}{2(2 \pi)^{D-1}} C_{\beta \alpha}^{-1} C_{\lambda \gamma} \int \frac{d^{D} k_{1}}{(2 \pi)^{D}} \\
& \times\left[\cos ^{2}\left(\frac{1}{2} k_{1} \wedge p_{2}\right) \widetilde{S}_{\beta \lambda}^{+}\left(k_{1}\right) \widetilde{S}_{\alpha \gamma}^{+}\left(-k_{1}-p_{2}\right)\right. \\
& \left.+\cos ^{2}\left(\frac{1}{2} k_{1} \wedge p_{1}\right) \widetilde{S}_{\beta \lambda}^{+}\left(k_{1}\right) \widetilde{S}_{\alpha \gamma}^{+}\left(-k_{1}+p_{1}\right)\right] .
\end{aligned}
$$

Segue portanto que a condição

$$
2 \operatorname{Re}\left\langle p_{1}\left|A_{2}\right| p_{2}\right\rangle=\left\langle p_{1}\left|A_{1}^{\dagger} A_{1}\right| p_{2}\right\rangle
$$

também é satisfeita para o diagrama da figura (5.2). Com esse resultado fica concluída a análise da unitariedade a um laço e segunda ordem na constante de acoplamento no modelo de Yukawa, pois analisamos os dois diagramas próprios que contribuem para a matriz $S$ até a ordem considerada. Apenas para ressaltar, em nossa análise não foi preciso tratar os casos de não comutatividade espacial e não comutatividade espaço-temporal separadamente, ou seja, os resultados estabelecidos valem independentemente disso. 


\section{Considerações Finais e Perspectivas}

Ao longo deste trabalho temos discutido a questão da unitariedade no caso de teorias não comutativas. A condição de unitariedade é um dos requerimentos fundamentais para a interpretação quântica de uma teoria. O estudo foi iniciado com o desenvolvimento de regras bem definidas (regras de corte) na situação comutativa, que permitem uma análise sistemática da unitariedade. Na sequência, introduzimos a não comutatividade no contexto do produto Moyal e discutimos suas propriedades básicas. Em particular, citamos o fato que as partes quadráticas da ação não se alteram na presença do produto Moyal, tal que seus efeitos manifestam-se apenas na parte relativa à interação. Isso sugere que ainda podemos usar teoria de perturbação via regras de Feynman (modificadas). Essas idéias foram desenvolvidas no segundo capítulo, em que investigamos uma relação entre diagramas do modelo $\phi^{3}$ fornecida pelas regras de corte. Como resultado, verificamos uma violação da unitariedade quando a não comutatividade envolve o espaço e o tempo $\left(\Theta_{0 i} \neq 0\right)$. Isso mostra que a simples aplicação das regras de Feynman, apenas levando em conta os fatores oriundos do produto Moyal, não é suficiente no caso de teorias não locais no tempo.

Para tratar o problema mencionado, partimos para uma abordagem diferente. De acordo com essa proposta, a idéia é, por um lado, escrever soluções iterativas para os campos satifazendo a equação de Klein-Gordon. Assim, obtemos o campo assintótico emergente, $\phi_{e m}$, como potências do campo assintótico incidente, $\phi_{i n}$. Por outro lado, definimos uma matriz $S$ com a prescrição da realização minimal, a qual pode ser exemplificada como segue: dado um diagrama com $n$ linhas ligando dois vértices (no modelo $\phi^{3}$ não é possível ter mais que duas), o ordenamento temporal é realizado em apenas uma delas. A realização minimal é suficiente para que a matriz $S$ conecte corretamente os campos $\phi_{i n}$ e $\phi_{e m}$ por meio da relação $\phi_{e m}=S^{\dagger} \phi_{i n} S$. Corretamente significa que os campos obtidos daí são justamente iguais aos campos obtidos via solução iterativa. Além disso, a unitariedade da matriz $S$ é verificada perturbativamente. Como aplicação desses métodos, retornamos ao estudo do modelo $\phi^{3}$, que havia apresentado problemas anteriormente. Como esperado, a unitariedade a um laço e segunda ordem na constante de acoplamento foi verificada independentemente se a não comutatividade envolve o espaço e tempo ou apenas o espaço.

Os desenvolvimentos anteriores foram feitos com campos escalares. A sequência na- 
tural é o estudo envolvendo campos fermiônicos. Assim, estendemos o método anterior ao modelo de Yukawa, cuja interação é da forma $\bar{\psi} * \psi * \phi$. Mais precisamente, estudamos diagramas de um laço que, dentro do esquema do segundo capítulo apresentariam violação da unitariedade quando $\Theta_{0 i} \neq 0$, pois os ingredientes que levam a essa quebra estão presentes também nesses gráficos. Entretanto, com o método descrito no terceiro capítulo, verificamos que a relação de unitariedade da matriz $S$, relativa aos diagrama considerados, é satisfeita. Pretendemos prosseguir com essa investigação além de campos fermiônicos, considerando o caso de teorias de gauge.

Apesar do método apresentado não sofrer da violação da unitariedade, pelo menos nos diagramas estudados, existem outras propostas interessantes. Em particular, destacamos a referência [24], em que $\Theta_{\mu \nu}$ é tratado como um tensor de segunda ordem. Além da unitariedade preservada, a simetria de Lorentz não é quebrada. Pretendemos analisar também esse tipo de tratamento. 


\section{Apêndice A}

\section{Propagador anti-ordenado temporalmente}

Queremos mostrar o resultado (1.11) partindo de (1.10). Para esse fim, usaremos a representação integral para a função $\theta$,

$$
\theta\left(x^{0}\right)=-\frac{1}{2 \pi i} \int_{-\infty}^{\infty} d \tau \frac{e^{-i \tau x^{0}}}{\tau+i \epsilon} .
$$

Além disso, vamos empregar a propriedade

$$
\delta(f(x))=\sum_{i} \frac{\delta\left(x-x_{0 i}\right)}{\left|\left(\frac{d f}{d x}\right)_{x_{0 i}}\right|}
$$

com $x_{0 i}$ representando as raízes da função $f(x)$, para escrever o termo $\theta\left(p^{0}\right) \delta\left(p^{2}-m^{2}\right)$ como

$$
\begin{aligned}
\theta\left(p^{0}\right) \delta\left(p^{2}-m^{2}\right) & =\theta\left(p^{0}\right)\left(\frac{\delta\left(p^{0}-\omega_{p}\right)}{2 \omega_{p}}+\frac{\delta\left(p^{0}+\omega_{p}\right)}{2 \omega_{p}}\right) \\
& =\frac{\delta\left(p^{0}-\omega_{q}\right)}{2 \omega_{p}}
\end{aligned}
$$

sendo $\omega_{p} \equiv \sqrt{\mathbf{p}^{2}+m^{2}}$. Da mesma forma,

$$
\theta\left(-p^{0}\right) \delta\left(p^{2}-m^{2}\right)=\frac{\delta\left(p^{0}+\omega_{q}\right)}{2 \omega_{p}}
$$

Com esses resultados, escrevemos (1.10) da seguinte maneira, 


$$
\bar{\Delta}(x)=-\frac{1}{2 \pi i} \int \frac{d^{3} p}{(2 \pi)^{3}} \int \frac{d \tau}{2 \omega_{p}} \frac{e^{-i\left(\tau-\omega_{p}\right) x^{0}+i \mathbf{p} \cdot \mathbf{x}}}{\tau+i \epsilon}-\frac{1}{2 \pi i} \int \frac{d^{3} p}{(2 \pi)^{3}} \int \frac{d \tau}{2 \omega_{p}} \frac{e^{i\left(\tau-\omega_{p}\right) x^{0}+i \mathbf{p} \cdot \mathbf{x}}}{\tau+i \epsilon} .
$$

Nessa equação já foi calculada a integral em $p^{0}$ com a função delta. Agora, fazemos a mudança de variável $\gamma \equiv \tau-\omega_{p}$, resultando

$$
\bar{\Delta}(x)=-\frac{1}{2 \pi i} \int \frac{d^{3} p}{(2 \pi)^{3}} \int \frac{d \gamma}{2 \omega_{p}} \frac{e^{-i \gamma x^{0}+i \mathbf{p} \cdot \mathbf{x}}}{\gamma+\omega_{p}+i \epsilon}-\frac{1}{2 \pi i} \int \frac{d^{3} p}{(2 \pi)^{3}} \int \frac{d \gamma}{2 \omega_{p}} \frac{e^{i \gamma x^{0}+i \mathbf{p} \cdot \mathbf{x}}}{\gamma+\omega_{p}+i \epsilon} .
$$

Fazendo $\gamma \rightarrow-\gamma$ no segundo termo do lado direito, segue que

$$
\begin{aligned}
\bar{\Delta}(x) & =-\frac{1}{2 \pi i} \int \frac{d^{3} p}{(2 \pi)^{3}} \int \frac{d \gamma}{2 \omega_{p}} e^{-i \gamma x^{0}+i \mathbf{p} \cdot \mathbf{x}}\left(\frac{1}{\gamma+\omega_{p}+i \epsilon}+\frac{1}{-\gamma+\omega_{p}+i \epsilon}\right) \\
& =\int \frac{d^{4} p}{(2 \pi)^{4}} \frac{-i}{p^{2}-m^{2}-i \epsilon} e^{-i p x} .
\end{aligned}
$$

Na última passagem apenas redefinimos o parâmetro infinitesimal $\epsilon$ e tomamos o limite $\epsilon \rightarrow 0$ no numerador. Também identificamos $\gamma \equiv p^{0}$. Assim, mostramos o resultado desejado. 


\section{Apêndice B}

\section{Produto Moyal envolvendo campos iguais}

Neste apêndice são mostrados os cálculos referentes aos resultados $(1.46),(1.47),(1.48)$ e (1.49), que surgem quando alguns dos campos que participam do produto Moyal são iguais. O resultado (1.46) é obtido como segue:

$$
\begin{aligned}
& \int d^{D} x \phi_{1}(x) * \phi_{1}(x) * \phi_{2}(x) * \phi_{2}(x) \\
& =\int \prod_{i=1}^{4} \frac{d^{D} k_{i}}{(2 \pi)^{D}}(2 \pi)^{D} \delta^{D}\left(k_{1}+k_{2}+k_{3}+k_{4}\right) e^{-\frac{i}{2}\left(k_{1} \wedge k_{2}+k_{1} \wedge k_{3}+k_{1} \wedge k_{4}+k_{2} \wedge k_{3}+k_{2} \wedge k_{4}+k_{3} \wedge k_{4}\right)} \\
& \times \widetilde{\phi}_{1}\left(k_{1}\right) \widetilde{\phi}_{1}\left(k_{2}\right) \widetilde{\phi}_{2}\left(k_{3}\right) \widetilde{\phi}_{2}\left(k_{4}\right) \\
& =\int \prod_{i=1}^{4} \frac{d^{D} k_{i}}{(2 \pi)^{D}}(2 \pi)^{D} \delta^{D}\left(k_{1}+k_{2}+k_{3}+k_{4}\right) e^{-\frac{i}{2} k_{1} \wedge k_{2}} e^{-\frac{i}{2} k_{3} \wedge k_{4}} \widetilde{\phi}_{1}\left(k_{1}\right) \widetilde{\phi}_{1}\left(k_{2}\right) \widetilde{\phi}_{2}\left(k_{3}\right) \widetilde{\phi}_{2}\left(k_{4}\right) \\
& =\int \prod_{i=1}^{4} \frac{d^{D} k_{i}}{(2 \pi)^{D}}(2 \pi)^{D} \delta^{D}\left(k_{1}+k_{2}+k_{3}+k_{4}\right)\left(\frac{e^{-\frac{i}{2} k_{1} \wedge k_{2}}+e^{-\frac{i}{2} k_{2} \wedge k_{1}}}{2}\right) \\
& \times\left(\frac{e^{-\frac{i}{2} k_{3} \wedge k_{4}}+e^{-\frac{i}{2} k_{4} \wedge k_{3}}}{2}\right) \widetilde{\phi}_{1}\left(k_{1}\right) \widetilde{\phi}_{1}\left(k_{2}\right) \widetilde{\phi}_{2}\left(k_{3}\right) \widetilde{\phi}_{2}\left(k_{4}\right) \\
& =\int \prod_{i=1}^{4} \frac{d^{D} k_{i}}{(2 \pi)^{D}}(2 \pi)^{D} \delta^{D}\left(k_{1}+k_{2}+k_{3}+k_{4}\right) \cos \left(\frac{k_{1} \wedge k_{2}}{2}\right) \cos \left(\frac{k_{3} \wedge k_{4}}{2}\right) \\
& \times \widetilde{\phi}_{1}\left(k_{1}\right) \widetilde{\phi}_{1}\left(k_{2}\right) \widetilde{\phi}_{2}\left(k_{3}\right) \widetilde{\phi}_{2}\left(k_{4}\right) \text {. }
\end{aligned}
$$

Agora, vamos ao resultado (1.47); 


$$
\begin{aligned}
& \int d^{D} x \phi_{1}(x) * \phi_{2}(x) * \phi_{1}(x) * \phi_{2}(x) \\
= & \int \prod_{i=1}^{4} \frac{d^{D} k_{i}}{(2 \pi)^{D}}(2 \pi)^{D} \delta^{D}\left(k_{1}+k_{2}+k_{3}+k_{4}\right) e^{-\frac{i}{2}\left(k_{1} \wedge k_{2}+k_{1} \wedge k_{3}+k_{2} \wedge k_{3}\right)} \\
\times & \widetilde{\phi}_{1}\left(k_{1}\right) \widetilde{\phi}_{2}\left(k_{2}\right) \widetilde{\phi}_{1}\left(k_{3}\right) \widetilde{\phi}_{2}\left(k_{4}\right) \\
= & \int \prod_{i=1}^{4} \frac{d^{D} k_{i}}{(2 \pi)^{D}}(2 \pi)^{D} \delta^{D}\left(k_{1}+k_{2}+k_{3}+k_{4}\right) \\
\times & \frac{e^{-\frac{i}{2}\left(k_{1} \wedge k_{2}+k_{1} \wedge k_{3}+k_{2} \wedge k_{3}\right)}+e^{-\frac{i}{2}\left(k_{3} \wedge k_{2}+k_{3} \wedge k_{1}+k_{2} \wedge k_{1}\right)}}{2} \widetilde{\phi}_{1}\left(k_{1}\right) \widetilde{\phi}_{2}\left(k_{2}\right) \widetilde{\phi}_{1}\left(k_{3}\right) \widetilde{\phi}_{2}\left(k_{4}\right) \\
= & \int \prod_{i=1}^{4} \frac{d^{D} k_{i}}{(2 \pi)^{D}}(2 \pi)^{D} \delta^{D}\left(k_{1}+k_{2}+k_{3}+k_{4}\right) \cos \left(\frac{k_{1} \wedge k_{2}+k_{1} \wedge k_{3}+k_{2} \wedge k_{3}}{2}\right) \\
\times & \widetilde{\phi}_{1}\left(k_{1}\right) \widetilde{\phi}_{2}\left(k_{2}\right) \widetilde{\phi}_{1}\left(k_{3}\right) \widetilde{\phi}_{2}\left(k_{4}\right) \\
= & \int \prod_{i=1}^{4} \frac{d^{D} k_{i}}{(2 \pi)^{D}}(2 \pi)^{D} \delta^{D}\left(k_{1}+k_{2}+k_{3}+k_{4}\right) \cos \left(\frac{k_{1} \wedge k_{2}+k_{3} \wedge k_{4}}{2}\right) \\
\times & \widetilde{\phi}_{1}\left(k_{1}\right) \widetilde{\phi}_{2}\left(k_{2}\right) \widetilde{\phi}_{1}\left(k_{3}\right) \widetilde{\phi}_{2}\left(k_{4}\right),
\end{aligned}
$$

sendo que na última passagem eliminamos o segundo $k_{2}$ no argumento do co-seno via delta, quer dizer $k_{2}=-\left(k_{1}+k_{3}+k_{4}\right)$.

Como explicado no texto, devemos tratar os campos fermiônicos clássicos como variáveis de Grassmann. Desse modo, o resultado (1.48) é obtido da seguinte maneira; 


$$
\begin{aligned}
& \int d^{D} x \bar{\psi}_{1}(x) * \bar{\psi}_{1}(x) * \psi_{2}(x) * \psi_{2}(x) \\
= & \int \prod_{i=1}^{4} \frac{d^{D} k_{i}}{(2 \pi)^{D}}(2 \pi)^{D} \delta^{D}\left(k_{1}+k_{2}+k_{3}+k_{4}\right) e^{-\frac{i}{2}\left(k_{1} \wedge k_{2}+k_{3} \wedge k_{4}\right)} \widetilde{\bar{\psi}}_{1}\left(k_{1}\right) \widetilde{\bar{\psi}}_{1}\left(k_{2}\right) \widetilde{\psi}_{2}\left(k_{3}\right) \widetilde{\psi}_{2}\left(k_{4}\right) \\
= & \int \prod_{i=1}^{4} \frac{d^{D} k_{i}}{(2 \pi)^{D}}(2 \pi)^{D} \delta^{D}\left(k_{1}+k_{2}+k_{3}+k_{4}\right) \\
\times & \frac{e^{-\frac{i}{2} k_{1} \wedge k_{2}} \widetilde{\bar{\psi}}_{1}\left(k_{1}\right) \widetilde{\bar{\psi}}_{1}\left(k_{2}\right)+e^{-\frac{i}{2} k_{2} \wedge k_{1}} \widetilde{\bar{\psi}}_{1}\left(k_{2}\right) \widetilde{\bar{\psi}}_{1}\left(k_{1}\right)}{2} \\
\times & \frac{e^{-\frac{i}{2} k_{3} \wedge k_{4}} \widetilde{\psi}_{2}\left(k_{3}\right) \widetilde{\psi}_{2}\left(k_{4}\right)+e^{-\frac{i}{2} k_{4} \wedge k_{3}} \widetilde{\psi}_{2}\left(k_{4}\right) \widetilde{\psi}_{2}\left(k_{3}\right)}{2} \\
= & \int \prod_{i=1}^{4} \frac{d^{D} k_{i}}{(2 \pi)^{D}}(2 \pi)^{D} \delta^{D}\left(k_{1}+k_{2}+k_{3}+k_{4}\right) \\
\times & \frac{e^{-\frac{i}{2} k_{1} \wedge k_{2}} \widetilde{\bar{\psi}}_{1}\left(k_{1}\right) \widetilde{\bar{\psi}}_{1}\left(k_{2}\right)-e^{\frac{i}{2} k_{1} \wedge k_{2}} \widetilde{\bar{\psi}}_{1}\left(k_{1}\right) \widetilde{\bar{\psi}}_{1}\left(k_{2}\right)}{2} \\
\times & \frac{e^{-\frac{i}{2} k_{3} \wedge k_{4}} \widetilde{\psi}_{2}\left(k_{3}\right) \widetilde{\psi}_{2}\left(k_{4}\right)-e^{\frac{i}{2} k_{3} \wedge k_{4}} \widetilde{\psi}_{2}\left(k_{3}\right) \widetilde{\psi}_{2}\left(k_{4}\right)}{2} \\
= & -\int \prod_{i=1}^{4} \frac{d k_{i}}{(2 \pi)^{D}}(2 \pi)^{D} \delta^{D}\left(k_{1}+k_{2}+k_{3}+k_{4}\right) \sin \left(\frac{k_{1} \wedge k_{2}}{2}\right) \sin \left(\frac{k_{3} \wedge k_{4}}{2}\right) \\
\times & \widetilde{\bar{\psi}}_{1}\left(k_{1}\right) \widetilde{\bar{\psi}}_{1}\left(k_{2}\right) \widetilde{\psi}_{2}\left(k_{3}\right) \widetilde{\psi}_{2}\left(k_{4}\right) .
\end{aligned}
$$

Por fim, o resultado (1.49) é obtido da seguinte forma: 


$$
\begin{aligned}
& \int d^{D} x \bar{\psi}_{1}(x) * \psi_{2}(x) * \bar{\psi}_{1}(x) * \psi_{2}(x) \\
& =\int \prod_{i=1}^{4} \frac{d^{D} k_{i}}{(2 \pi)^{D}}(2 \pi)^{D} \delta^{D}\left(k_{1}+k_{2}+k_{3}+k_{4}\right) \\
& \times \frac{1}{2}\left[e^{-\frac{i}{2}\left(k_{1} \wedge k_{2}+k_{1} \wedge k_{3}+k_{2} \wedge k_{3}\right)} \widetilde{\bar{\psi}}_{1}\left(k_{1}\right) \widetilde{\psi}_{2}\left(k_{2}\right) \widetilde{\bar{\psi}}_{1}\left(k_{3}\right) \widetilde{\psi}_{2}\left(k_{4}\right)\right. \\
& \left.+e^{-\frac{i}{2}\left(k_{3} \wedge k_{2}+k_{3} \wedge k_{1}+k_{2} \wedge k_{1}\right)} \widetilde{\bar{\psi}}_{1}\left(k_{3}\right) \widetilde{\psi}_{2}\left(k_{2}\right) \widetilde{\bar{\psi}}_{1}\left(k_{1}\right) \widetilde{\psi}_{2}\left(k_{4}\right)\right] \\
& =-i \int \prod_{i=1}^{4} \frac{d^{D} k_{i}}{(2 \pi)^{D}}(2 \pi)^{D} \delta^{D}\left(k_{1}+k_{2}+k_{3}+k_{4}\right) \sin \left(\frac{k_{1} \wedge k_{2}+k_{3} \wedge k_{4}}{2}\right) \\
& \times \widetilde{\bar{\psi}}_{1}\left(k_{1}\right) \widetilde{\psi}_{2}\left(k_{2}\right) \widetilde{\bar{\psi}}_{1}\left(k_{3}\right) \widetilde{\psi}_{2}\left(k_{4}\right) \text {, }
\end{aligned}
$$

em que usamos $k_{2}=-\left(k_{1}+k_{3}+k_{4}\right)$ tal como em (B.2). 


\section{Apêndice $\mathrm{C}$}

\section{Férmions de Majorana}

Consideremos os espinores de Dirac

$$
\psi_{\alpha}(x)=\frac{1}{(2 \pi)^{\frac{D-1}{2}}} \sum_{r} \int \frac{d^{D-1} p}{\omega_{p} / M}\left[a_{p}^{(r)} u_{\alpha}^{(r)}(p) e^{-i p x}+b_{p}^{\dagger(r)} v_{\alpha}^{(r)}(p) e^{i p x}\right]
$$

e

$$
\bar{\psi}_{\alpha}(x)=\frac{1}{(2 \pi)^{\frac{D-1}{2}}} \sum_{r} \int \frac{d^{D-1} p}{\omega_{p} / M}\left[a_{p}^{\dagger(r)} \bar{u}_{\alpha}^{(r)}(p) e^{i p x}+b_{p}^{(r)} \bar{v}_{\alpha}^{(r)}(p) e^{-i p x}\right],
$$

com $\bar{\psi} \equiv \psi^{\dagger} \gamma^{0}$. Os operadores $a_{p}^{(r)}, a_{p}^{\dagger(r)}, b_{p}^{(r)}$ e $b_{p}^{\dagger(r)}$ satisfazem as seguintes relações de anti-comutação:

$$
\left\{a_{p}^{(r)}, a_{q}^{\dagger(s)}\right\}=\left\{b_{p}^{(r)}, b_{q}^{\dagger(s)}\right\}=\frac{\omega_{p}}{M} \delta_{r s} \delta^{D-1}(\mathbf{p}-\mathbf{q}),
$$

sendo zero todas outras. $u$ e $v$ são tais que

$$
\left(p_{\mu} \gamma^{\mu}-M\right) u^{(r)}(p)=0
$$

e

$$
\left(p_{\mu} \gamma^{\mu}+M\right) v^{(r)}(p)=0 .
$$

O índice $r$ indica a polarização, ou seja, a projeção do spin.

Partindo dos espinores de Dirac, um espinor de Majorana pode ser obtido por meio de,

$$
\psi_{M}=\frac{1}{\sqrt{2}}\left(\psi^{c}+\psi\right)
$$


em que $\psi^{c}$ é o campo conjugado de carga; $\psi^{c} \equiv \bar{\psi} C$. C é a matriz de conjugação de carga, a qual satisfaz algumas propriedades,

$$
C=-C^{T}=-C^{-1}=-C^{\dagger} .
$$

Além dessas, sua aplicação sobre $\bar{u}^{(r)}$ e $\bar{v}^{(r)}$, resulta em

$$
u^{(r)}(p)=\bar{v}^{(r)}(p) C
$$

e

$$
v^{(r)}(p)=\bar{u}^{(r)}(p) C
$$

Antes de substituir os campos em (C.6), calculemos $\psi^{c}$;

$$
\begin{aligned}
\psi_{\alpha}^{c}(x) & =\frac{1}{(2 \pi)^{\frac{D-1}{2}}} \sum_{r} \int \frac{d^{D-1} p}{\omega_{p} / M}\left[a_{p}^{\dagger(r)} \bar{u}_{\alpha}^{(r)}(p) C e^{i p x}+b_{p}^{(r)} \bar{v}_{\alpha}^{(r)}(p) C e^{-i p x}\right] \\
& =\frac{1}{(2 \pi)^{\frac{D-1}{2}}} \sum_{r} \int \frac{d^{D-1} p}{\omega_{p} / M}\left[a_{p}^{\dagger(r)} v_{\alpha}^{(r)}(p) e^{i p x}+b_{p}^{(r)} u_{\alpha}^{(r)}(p) e^{-i p x}\right]
\end{aligned}
$$

Substituindo (C.1) e (C.10) em (C.6), obtemos

$$
\psi_{\alpha M}(x)=\frac{1}{(2 \pi)^{\frac{D-1}{2}}} \sum_{r} \int \frac{d^{D-1} p}{\omega_{p} / M}\left[c_{p}^{(r)} u_{\alpha}^{(r)}(p) e^{-i p x}+c_{p}^{\dagger(r)} v_{\alpha}^{(r)}(p) e^{i p x}\right],
$$

em que identificamos

$$
c_{p}^{(r)} \equiv \frac{1}{\sqrt{2}}\left(a_{p}^{(r)}+b_{p}^{(r)}\right) .
$$

A relação de anti-comutação entre $c_{p}^{(r)}$ e $c_{p}^{\dagger(r)}$ é

$$
\left\{c_{p}^{(r)}, c_{q}^{\dagger(s)}\right\}=\frac{\omega_{p}}{M} \delta_{r s} \delta^{D-1}(\mathbf{p}-\mathbf{q})
$$




\section{Apêndice D}

\section{Produto Moyal com campos fermiônicos de Majorana}

Neste apêndice deduziremos a expressão (5.6). Essa propriedade pode ser demonstrada partindo da equação (1.44), porém com campos fermiônicos de Majorana clássicos;

$$
\begin{aligned}
& \int d^{D} x \bar{\psi}_{\alpha}(x) * \psi_{\alpha}(x) * \phi(x) \\
= & \int \frac{d^{D} k_{1}}{(2 \pi)^{D}} \frac{d^{D} k_{2}}{(2 \pi)^{D}} \frac{d^{D} k_{3}}{(2 \pi)^{D}}(2 \pi)^{D} \delta^{D}\left(k_{1}+k_{2}+k_{3}\right) e^{-\frac{i}{2} k_{1} \wedge k_{2}} \widetilde{\bar{\psi}}_{\alpha}\left(k_{1}\right) \widetilde{\psi}_{\alpha}\left(k_{2}\right) \widetilde{\phi}\left(k_{3}\right) \\
= & \int \frac{d^{D} k_{1}}{(2 \pi)^{D}} \frac{d^{D} k_{2}}{(2 \pi)^{D}} \frac{d^{D} k_{3}}{(2 \pi)^{D}}(2 \pi)^{D} \delta^{D}\left(k_{1}+k_{2}+k_{3}\right) e^{-\frac{i}{2} k_{1} \wedge k_{2}} \widetilde{\psi}_{\beta}\left(k_{1}\right) \widetilde{\psi}_{\alpha}\left(k_{2}\right) \widetilde{\phi}\left(k_{3}\right) C_{\beta \alpha}^{-1} \\
= & \int \frac{d^{D} k_{1}}{(2 \pi)^{D}} \frac{d^{D} k_{2}}{(2 \pi)^{D}} \frac{d^{D} k_{3}}{(2 \pi)^{D}}(2 \pi)^{D} \delta^{D}\left(k_{1}+k_{2}+k_{3}\right) \\
\times & \frac{1}{2}\left[e^{-\frac{i}{2} k_{1} \wedge k_{2}} \widetilde{\psi}_{\beta}\left(k_{1}\right) \widetilde{\psi}_{\alpha}\left(k_{2}\right)+e^{-\frac{i}{2} k_{2} \wedge k_{1}} \widetilde{\psi}_{\beta}\left(k_{2}\right) \widetilde{\psi}_{\alpha}\left(k_{1}\right)\right] \widetilde{\phi}\left(k_{3}\right) C_{\beta \alpha}^{-1} \\
= & \int \frac{d^{D} k_{1}}{(2 \pi)^{D}} \frac{d^{D} k_{2}}{(2 \pi)^{D}} \frac{d^{D} k_{3}}{(2 \pi)^{D}}(2 \pi)^{D} \delta^{D}\left(k_{1}+k_{2}+k_{3}\right) \\
\times & \frac{1}{2}\left[e^{-\frac{i}{2} k_{1} \wedge k_{2}} \widetilde{\psi}_{\beta}\left(k_{1}\right) \widetilde{\psi}_{\alpha}\left(k_{2}\right)-e^{-\frac{i}{2} k_{2} \wedge k_{1}} \widetilde{\psi}_{\alpha}\left(k_{1}\right) \widetilde{\psi}_{\beta}\left(k_{2}\right)\right] \widetilde{\phi}\left(k_{3}\right) C_{\beta \alpha}^{-1} \\
= & \int \frac{d^{D} k_{1}}{(2 \pi)^{D}} \frac{d^{D} k_{2}}{(2 \pi)^{D}} \frac{d^{D} k_{3}}{(2 \pi)^{D}}(2 \pi)^{D} \delta^{D}\left(k_{1}+k_{2}+k_{3}\right) \\
\times & \frac{1}{2}\left[e^{-\frac{i}{2} k_{1} \wedge k_{2}} \widetilde{\bar{\psi}}_{\alpha}\left(k_{1}\right) \widetilde{\psi}_{\alpha}\left(k_{2}\right)+e^{\frac{i}{2} k_{1} \wedge k_{1}} \widetilde{\bar{\psi}}_{\alpha}\left(k_{1}\right) \widetilde{\psi}_{\alpha}\left(k_{2}\right)\right] \widetilde{\phi}\left(k_{3}\right)
\end{aligned}
$$




$$
=\int \frac{d^{D} k_{1}}{(2 \pi)^{D}} \frac{d^{D} k_{2}}{(2 \pi)^{D}} \frac{d^{D} k_{3}}{(2 \pi)^{D}}(2 \pi)^{D} \delta^{D}\left(k_{1}+k_{2}+k_{3}\right) \cos \left(\frac{k_{1} \wedge k_{2}}{2}\right) \widetilde{\bar{\psi}}_{\alpha}\left(k_{1}\right) \widetilde{\psi}_{\alpha}\left(k_{2}\right) \widetilde{\phi}\left(k_{3}\right) .
$$

Nesse cálculo usamos a propriedade $C=-C^{T}$, além da relação $\psi=\bar{\psi} C$. 


\section{Apêndice E}

\section{Demonstração de que os produtos matriciais em (5.32) são reais}

Vamos provar que os produtos matriciais

$$
\bar{u}_{\alpha}^{\left(t_{1}\right)}\left(p_{1}\right) \widetilde{S}_{\alpha \gamma}^{+}\left(k_{1}\right) u_{\gamma}^{\left(t_{2}\right)}\left(p_{1}\right)
$$

e

$$
\bar{v}_{\alpha}^{\left(t_{2}\right)}\left(p_{2}\right) \widetilde{S}_{\alpha \gamma}^{+}\left(k_{1}\right) v_{\gamma}^{\left(t_{1}\right)}\left(p_{2}\right)
$$

são reais. Como $\widetilde{S}_{\alpha \gamma}^{+}\left(k_{1}\right)=\left(\gamma^{\mu} k_{1 \mu}+M\right)_{\alpha \beta}(2 \pi) \delta\left(k_{1}^{2}-M^{2}\right) \theta\left(k_{1}^{0}\right)$, temos que mostrar que os objetos $\bar{u}^{\left(t_{1}\right)}\left(p_{1}\right) \gamma^{\mu} u^{\left(t_{2}\right)}\left(p_{1}\right), \bar{u}^{\left(t_{1}\right)}\left(p_{1}\right) u^{\left(t_{2}\right)}\left(p_{1}\right), \bar{v}^{\left(t_{2}\right)}\left(p_{2}\right) \gamma^{\mu} v^{\left(t_{1}\right)}\left(p_{2}\right)$ e $\bar{v}^{\left(t_{2}\right)}\left(p_{2}\right) v^{\left(t_{1}\right)}\left(p_{2}\right)$ são reais. Dois entre esses são reais de partida, pois satisfazem as seguintes normalizações:

$$
\bar{u}^{\left(t_{1}\right)}\left(p_{1}\right) u^{\left(t_{2}\right)}\left(p_{1}\right)=\delta_{t_{1} t_{2}}
$$

$\mathrm{e}$

$$
\bar{v}^{\left(t_{2}\right)}\left(p_{2}\right) v^{\left(t_{1}\right)}\left(p_{2}\right)=-\delta_{t_{1} t_{2}} .
$$

Os demais podem ser provados via identidade de Gordon;

$$
\bar{u}^{(r)}(p) \gamma^{\mu} u^{(s)}(q)=\frac{\left(p^{\mu}+q^{\mu}\right)}{2 M} \bar{u}^{(r)}(p) u^{(s)}(q)-\bar{u}^{(r)}(p) \frac{\left[\gamma^{\mu}, \gamma^{\nu}\right]}{4 M} u^{(s)}(q)\left(p_{\nu}-q_{\nu}\right),
$$

a qual será demonstrada brevemente. Tomando $p=q=p_{1}$, segue que

$$
\bar{u}^{(r)}\left(p_{1}\right) \gamma^{\mu} u^{(s)}\left(p_{1}\right)=\frac{p_{1}^{\mu}}{M} \bar{u}^{(r)}\left(p_{1}\right) u^{(s)}\left(p_{1}\right),
$$


que claramente é real. Para o termo remanescente, $\bar{v}^{\left(t_{2}\right)}\left(p_{2}\right) \gamma^{\mu} v^{\left(t_{1}\right)}\left(p_{2}\right)$, usamos a identidade de Gordon relacionando $v$ e $\bar{v}$;

$$
\bar{v}^{(r)}(p) \gamma^{\mu} v^{(s)}(q)=-\frac{\left(p^{\mu}+q^{\mu}\right)}{2 M} \bar{v}^{(r)}(p) v^{(s)}(q)+\bar{v}^{(r)}(p) \frac{\left[\gamma^{\mu}, \gamma^{\nu}\right]}{4 M} v^{(s)}(q)\left(p_{\nu}-q_{\nu}\right)
$$

Tomando $p=q=p_{2}$, ficamos com

$$
\bar{v}^{(r)}\left(p_{2}\right) \gamma^{\mu} v^{(s)}\left(p_{2}\right)=-\frac{p_{2}^{\mu}}{M} \bar{v}^{(r)}\left(p_{2}\right) v^{(s)}\left(p_{2}\right)
$$

que também é real, conforme foi anunciado.

Agora, vamos à demonstração da identidade de Gordon (E.5). Partimos então do segundo termo do lado direito, isto é,

$$
\begin{aligned}
\bar{u}(p) \frac{\left[\gamma^{\mu}, \gamma^{\nu}\right]}{4 M} u(q)\left(p_{\nu}-q_{\nu}\right) & =\frac{1}{4 M} \bar{u}(p) \gamma^{\mu} \gamma^{\nu} p_{\nu} u(q)-\frac{1}{4 M} \bar{u}(p) \gamma^{\mu} \gamma^{\nu} q_{\nu} u(q) \\
& -\frac{1}{4 M} \bar{u}(p) p_{\nu} \gamma^{\nu} \gamma^{\mu} u(q)+\frac{1}{4 M} \bar{u}(p) q_{\nu} \gamma^{\nu} \gamma^{\mu} u(q)
\end{aligned}
$$

Estamos omitindo as polarizações. De acordo com

$$
\bar{u}(p)\left(p_{\mu} \gamma^{\mu}-M\right)=0
$$

e

$$
\left(p_{\mu} \gamma^{\mu}-M\right) u(p)=0
$$

podemos escrever o segundo e o terceiro termo de (E.9) como

$$
\begin{aligned}
-\frac{1}{4 M} \bar{u}(p) \gamma^{\mu} \gamma^{\nu} q_{\nu} u(q)-\frac{1}{4 M} \bar{u}(p) p_{\nu} \gamma^{\nu} \gamma^{\mu} u(q) & =-\frac{1}{4} \bar{u}(p) \gamma^{\mu} u(q)-\frac{1}{4} \bar{u}(p) \gamma^{\mu} u(q) \\
& =-\frac{1}{2} \bar{u}(p) \gamma^{\mu} u(q)
\end{aligned}
$$

Examinemos o primeiro termo de (E.9):

$$
\begin{aligned}
\frac{1}{4 M} \bar{u}(p) \gamma^{\mu} \gamma^{\nu} p_{\nu} u(q) & =\frac{1}{4 M} \bar{u}(p)\left(2 g^{\mu \nu}-\gamma^{\nu} \gamma^{\mu}\right) p_{\nu} u(q) \\
& =\frac{p^{\mu}}{2 M} \bar{u}(p) u(q)-\frac{1}{4} \bar{u}(p) \gamma^{\mu} u(q)
\end{aligned}
$$

em que usamos a álgebra de Dirac, $\left\{\gamma^{\mu}, \gamma^{\nu}\right\}=2 g^{\mu \nu}$ e a equação (E.10). Da mesma maneira, o último termo de (E.9) pode ser escrito como 


$$
\frac{1}{4 M} \bar{u}(p) q_{\nu} \gamma^{\nu} \gamma^{\mu} u(q)=\frac{q^{\mu}}{2 M} \bar{u}(p) u(q)-\frac{1}{4} \bar{u}(p) \gamma^{\mu} u(q)
$$

Substituindo os resultados (E.12), (E.13) e (E.14) em (E.9), chegamos à

$$
\bar{u}(p) \frac{\left[\gamma^{\mu}, \gamma^{\nu}\right]}{4 M} u(q)\left(p_{\nu}-q_{\nu}\right)=\frac{\left(p^{\mu}+q^{\mu}\right)}{2 M} \bar{u}(p) u(q)-\bar{u}(p) \gamma^{\mu} u(q)
$$

demonstrando assim a identidade de Gordon. A identidade para $v$ e $\bar{v}$ pode ser demonstrada do mesmo modo. 


\section{Apêndice F}

\section{Demonstração da igualdade $A_{1}^{\dagger}=A_{1}$ para o modelo de Yukawa}

Devemos calcular $(\bar{\psi}(x) * \psi(x) * \phi(x))^{\dagger}$. Vamos omitir os índices espinoriais. Então,

$$
\begin{aligned}
(\bar{\psi}(x) * \psi(x) * \phi(x))^{\dagger} & =e^{-\frac{i}{2}\left[\partial_{x_{1}} \wedge\left(\partial_{x_{2}}+\partial_{x_{3}}\right)+\partial_{x_{2}} \wedge \partial_{x_{3}}\right]} \phi\left(x_{3}\right) \psi^{\dagger}\left(x_{2}\right) \bar{\psi}^{\dagger}\left(x_{1}\right) \\
& =e^{-\frac{i}{2}\left[\partial_{x_{1}} \wedge\left(\partial_{x_{2}}+\partial_{x_{3}}\right)+\partial_{x_{2}} \wedge \partial_{x_{3}}\right]} \phi\left(x_{3}\right) \psi^{\dagger}\left(x_{2}\right) \gamma_{0}^{\dagger} \psi\left(x_{1}\right) \\
& =e^{-\frac{i}{2}\left[\partial_{x_{1}} \wedge\left(\partial_{x_{2}}+\partial_{x_{3}}\right)+\partial_{x_{2}} \wedge \partial_{x_{3}}\right]} \phi\left(x_{3}\right) \bar{\psi}\left(x_{2}\right) \psi\left(x_{1}\right) .
\end{aligned}
$$

Aqui, usamos o fato que $\gamma_{0}^{\dagger}=\gamma_{0}$. Fazendo as mudanças $x_{1} \leftrightarrow x_{3}$, obtemos

$$
(\bar{\psi}(x) * \psi(x) * \phi(x))^{\dagger}=\phi(x) * \bar{\psi}(x) * \psi(x) .
$$

Lembrando que em $A_{1}^{\dagger}$ há uma integração, fazemos uma permutação cíclica para estabelecer o resultado

$$
\begin{aligned}
A_{1}^{\dagger} & =\int d^{D} x(\bar{\psi}(x) * \psi(x) * \phi(x))^{\dagger} \\
& =\int d^{D} x \phi(x) * \bar{\psi}(x) * \psi(x) \\
& =\int d^{D} x \bar{\psi}(x) * \psi(x) * \phi(x) \\
& =A_{1} .
\end{aligned}
$$




\section{Apêndice G}

\section{Produto Moyal com prescrição minimal}

Investigaremos o produto Moyal presente na equação (5.43):

$$
\begin{aligned}
& \int d^{D} x \bar{\psi}_{\alpha}(x) *\left(e^{-i \omega x} \psi_{\alpha}(x)\right) * \phi(x) \\
= & \int \frac{d^{D} k_{1}}{(2 \pi)^{D}} \frac{d^{D} k_{2}}{(2 \pi)^{D}} \frac{d^{D} k_{3}}{(2 \pi)^{D}}(2 \pi)^{D} \delta^{D}\left(k_{1}+k_{2}+k_{3}\right) e^{-\frac{i}{2} k_{1} \wedge k_{2}} \widetilde{\bar{\psi}}_{\alpha}\left(k_{1}\right) \widetilde{\psi}_{\alpha}\left(k_{2}-\omega\right) \widetilde{\phi}\left(k_{3}\right) \\
= & C_{\beta \alpha}^{-1} \int \frac{d^{D} k_{1}}{(2 \pi)^{D}} \frac{d^{D} k_{2}}{(2 \pi)^{D}} \frac{d^{D} k_{3}}{(2 \pi)^{D}}(2 \pi)^{D} \delta^{D}\left(k_{1}+k_{2}+k_{3}\right) e^{-\frac{i}{2} k_{1} \wedge k_{2}} \widetilde{\psi}_{\beta}\left(k_{1}\right) \widetilde{\psi}_{\alpha}\left(k_{2}-\omega\right) \widetilde{\phi}\left(k_{3}\right) \\
= & C_{\beta \alpha}^{-1} \int \frac{d^{D} k_{1}}{(2 \pi)^{D}} \frac{d^{D} k_{2}}{(2 \pi)^{D}} \frac{d^{D} k_{3}}{(2 \pi)^{D}}(2 \pi)^{D} \delta^{D}\left(k_{1}+k_{2}+k_{3}\right) \\
\times & \frac{1}{2}\left[e^{-\frac{i}{2} k_{1} \wedge k_{2}} \widetilde{\psi}_{\beta}\left(k_{1}\right) \widetilde{\psi}_{\alpha}\left(k_{2}-\omega\right)+e^{\frac{i}{2} k_{1} \wedge k_{2}+\frac{i}{2} \omega \wedge\left(k_{1}+k_{2}\right)} \widetilde{\psi}_{\beta}\left(k_{2}-\omega\right) \widetilde{\psi}_{\alpha}\left(k_{1}\right)\right] \widetilde{\phi}\left(k_{3}\right) \\
= & C_{\beta \alpha}^{-1} \int \frac{d^{D} k_{1}}{(2 \pi)^{D}} \frac{d^{D} k_{2}}{(2 \pi)^{D}} \frac{d^{D} k_{3}}{(2 \pi)^{D}}(2 \pi)^{D} \delta^{D}\left(k_{1}+k_{2}+k_{3}\right) \\
\times & \frac{1}{2}\left[e^{-\frac{i}{2} k_{1} \wedge k_{2}} \widetilde{\psi}_{\beta}\left(k_{1}\right) \widetilde{\psi}_{\alpha}\left(k_{2}-\omega\right)-e^{\frac{i}{2} k_{1} \wedge k_{2}+\frac{i}{2} \omega \wedge\left(k_{1}+k_{2}\right)} \widetilde{\psi}_{\alpha}\left(k_{1}\right) \widetilde{\psi}_{\beta}\left(k_{2}-\omega\right)\right] \widetilde{\phi}\left(k_{3}\right) \\
= & \int \frac{d^{D} k_{1}}{(2 \pi)^{D}} \frac{d^{D} k_{2}}{(2 \pi)^{D}} \frac{d^{D} k_{3}}{(2 \pi)^{D}}(2 \pi)^{D} \delta^{D}\left(k_{1}+k_{2}+k_{3}\right) \\
\times & e^{\frac{i}{4} \omega \wedge\left(k_{1}+k_{2}\right)} \cos \left(\frac{1}{2} k_{1} \wedge k_{2}+\frac{1}{4} \omega \wedge\left(k_{1}+k_{2}\right)\right) \widetilde{\bar{\psi}}_{\alpha}\left(k_{1}\right) \widetilde{\psi}_{\alpha}\left(k_{2}-\omega\right) \widetilde{\phi}\left(k_{3}\right) .
\end{aligned}
$$

Na terceira igualdade simplesmente somamos um termo igual ao que já estava presente e dividimos por dois, porém nesse termo adicionado fizemos a mudança $k_{1} \leftrightarrow k_{2}-\omega$. 
Nas igualdades seguintes usamos a relação de anti-comutação entre os campos (clássicos) fermiônicos e as propriedades da matriz de conjugação de carga. 


\section{Apêndice $\mathbf{H}$}

\section{Cálculo de unitariedade no modelo de Yukawa}

Neste apêndice, efetuaremos o cálculo do lado direito da condição de unitariedade (5.2). As etapas são exatamente as mesmas que aquelas apresentadas no texto referente ao lado esquerdo de (5.2), porém sem a presença da função $\theta$ (e sem a realização minimal) o que o torna mais simples. Inicialmente temos,

$$
\begin{aligned}
\left\langle p_{1}\left|A_{1}^{\dagger} A_{1}\right| p_{2}\right\rangle & =\frac{\lambda^{2}}{4} \int d^{D} x d^{D} y\left\langle p_{1}\left|\mathcal{F}_{x y}\left[\bar{\psi}_{\alpha}(x) \psi_{\alpha}(x) \phi(x) \bar{\psi}_{\gamma}(y) \psi_{\gamma}(y) \phi(y)\right]\right| p_{2}\right\rangle \\
& =\frac{\lambda^{2}}{4} \int d^{D} x d^{D} y\left\langle p_{1}\left|\left(\bar{\psi}_{\alpha}(x) * \psi_{\alpha}(x) * \phi(x)\right)\left(\bar{\psi}_{\gamma}(y) * \psi_{\gamma}(y) * \phi(y)\right)\right| p_{2}\right\rangle
\end{aligned}
$$

Como não temos a presença da função $\theta$, podemos usar a relação (5.6):

$$
\begin{aligned}
\left\langle p_{1}\left|A_{1}^{\dagger} A_{1}\right| p_{2}\right\rangle & =\frac{\lambda^{2}}{4} \prod_{i=1}^{3} \int \frac{d^{D} k_{i}}{(2 \pi)^{D}} \prod_{j=1}^{3} \int \frac{d^{D} q_{j}}{(2 \pi)^{D}}(2 \pi)^{D} \delta\left(k_{1}+k_{2}+k_{3}\right) \\
& \times \cos \left(\frac{k_{1} \wedge k_{2}}{2}\right)(2 \pi)^{D} \delta\left(q_{1}+q_{2}+q_{3}\right) \cos \left(\frac{q_{1} \wedge q_{2}}{2}\right) \\
& \times\left\langle p_{1}\left|\widetilde{\bar{\psi}}_{\alpha}\left(k_{1}\right) \widetilde{\psi}_{\alpha}\left(k_{2}\right) \widetilde{\phi}\left(k_{3}\right) \widetilde{\bar{\psi}}_{\gamma}\left(q_{1}\right) \widetilde{\psi}_{\gamma}\left(q_{2}\right) \widetilde{\phi}\left(q_{3}\right)\right| p_{2}\right\rangle .
\end{aligned}
$$

Aplicando o teorema de Wick e selecionando apenas os termos que contribuem para o diagrama da figura (5.2), ficamos com 


$$
\begin{aligned}
\left\langle p_{1}\left|A_{1}^{\dagger} A_{1}\right| p_{2}\right\rangle & =\frac{\lambda^{2}}{4} \prod_{i=1}^{3} \int \frac{d^{D} k_{i}}{(2 \pi)^{D}} \prod_{j=1}^{3} \int \frac{d^{D} q_{j}}{(2 \pi)^{D}}(2 \pi)^{D} \delta\left(k_{1}+k_{2}+k_{3}\right) \\
& \times \cos \left(\frac{k_{1} \wedge k_{2}}{2}\right)(2 \pi)^{D} \delta\left(q_{1}+q_{2}+q_{3}\right) \cos \left(\frac{q_{1} \wedge q_{2}}{2}\right) \\
& \times 2 C_{\beta \alpha}^{-1} C_{\lambda \gamma}(2 \pi)^{D} \delta^{D}\left(k_{1}+q_{1}\right) \widetilde{S}_{\beta \lambda}^{+}\left(k_{1}\right)(2 \pi)^{D} \delta^{D}\left(k_{2}+q_{2}\right) \widetilde{S}_{\alpha \gamma}^{+}\left(k_{2}\right) \\
& \times\left\langle p_{1}\left|: \widetilde{\phi}\left(k_{3}\right) \widetilde{\phi}\left(q_{3}\right):\right| p_{2}\right\rangle .
\end{aligned}
$$

Usando o resultado (4.23) para o elemento de matriz, a equação acima torna-se

$$
\begin{aligned}
\left\langle p_{1}\left|A_{1}^{\dagger} A_{1}\right| p_{2}\right\rangle & =\frac{\lambda^{2}}{4} \prod_{i=1}^{3} \int \frac{d^{D} k_{i}}{(2 \pi)^{D}} \prod_{j=1}^{3} \int \frac{d^{D} q_{j}}{(2 \pi)^{D}}(2 \pi)^{D} \delta\left(k_{1}+k_{2}+k_{3}\right) \\
& \times \cos \left(\frac{k_{1} \wedge k_{2}}{2}\right)(2 \pi)^{D} \delta\left(q_{1}+q_{2}+q_{3}\right) \cos \left(\frac{q_{1} \wedge q_{2}}{2}\right) \\
& \times 2 C_{\beta \alpha}^{-1} C_{\lambda \gamma}(2 \pi)^{D} \delta^{D}\left(k_{1}+q_{1}\right) \widetilde{S}_{\beta \lambda}^{+}\left(k_{1}\right)(2 \pi)^{D} \delta^{D}\left(k_{2}+q_{2}\right) \widetilde{S}_{\alpha \gamma}^{+}\left(k_{2}\right) \\
& \times \frac{1}{(2 \pi)^{D-1}}\left[(2 \pi)^{D} \delta^{D}\left(k_{3}-p_{2}\right)(2 \pi)^{D} \delta^{D}\left(q_{3}+p_{1}\right)\right. \\
& \left.+(2 \pi)^{D} \delta^{D}\left(k_{3}+p_{1}\right)(2 \pi)^{D} \delta^{D}\left(q_{3}-p_{2}\right)\right] .
\end{aligned}
$$

Agora, basta calcular as integrais usando as funções delta. Primeiro, em $q_{1}$ e $q_{2}$ :

$$
\begin{aligned}
\left\langle p_{1}\left|A_{1}^{\dagger} A_{1}\right| p_{2}\right\rangle & =\frac{\lambda^{2}}{2(2 \pi)^{D-1}} C_{\beta \alpha}^{-1} C_{\lambda \gamma} \prod_{i=1}^{3} \int \frac{d^{D} k_{i}}{(2 \pi)^{D}} \int \frac{d^{D} q_{3}}{(2 \pi)^{D}}(2 \pi)^{D} \delta\left(k_{1}+k_{2}+k_{3}\right) \\
& \times \cos ^{2}\left(\frac{k_{1} \wedge k_{2}}{2}\right)(2 \pi)^{D} \delta\left(-k_{1}-k_{2}+q_{3}\right) \widetilde{S}_{\beta \lambda}^{+}\left(k_{1}\right) \widetilde{S}_{\alpha \gamma}^{+}\left(k_{2}\right) \\
& \times\left[(2 \pi)^{D} \delta^{D}\left(k_{3}-p_{2}\right)(2 \pi)^{D} \delta^{D}\left(q_{3}+p_{1}\right)\right. \\
& \left.+(2 \pi)^{D} \delta^{D}\left(k_{3}+p_{1}\right)(2 \pi)^{D} \delta^{D}\left(q_{3}-p_{2}\right)\right] .
\end{aligned}
$$

Integral em $q_{3}$; 


$$
\begin{aligned}
\left\langle p_{1}\left|A_{1}^{\dagger} A_{1}\right| p_{2}\right\rangle & =\frac{\lambda^{2}}{2(2 \pi)^{D-1}} C_{\beta \alpha}^{-1} C_{\lambda \gamma} \prod_{i=1}^{3} \int \frac{d^{D} k_{i}}{(2 \pi)^{D}}(2 \pi)^{D} \delta\left(k_{1}+k_{2}+k_{3}\right) \\
& \times \cos ^{2}\left(\frac{k_{1} \wedge k_{2}}{2}\right) \widetilde{S}_{\beta \lambda}^{+}\left(k_{1}\right) \widetilde{S}_{\alpha \gamma}^{+}\left(k_{2}\right) \\
& \times\left[(2 \pi)^{D} \delta^{D}\left(k_{3}-p_{2}\right)(2 \pi)^{D} \delta^{D}\left(k_{1}+k_{2}+p_{1}\right)\right. \\
& \left.+(2 \pi)^{D} \delta^{D}\left(k_{3}+p_{1}\right)(2 \pi)^{D} \delta^{D}\left(k_{1}+k_{2}-p_{2}\right)\right] .
\end{aligned}
$$

Integral em $k_{3}$;

$$
\begin{aligned}
\left\langle p_{1}\left|A_{1}^{\dagger} A_{1}\right| p_{2}\right\rangle & =\frac{\lambda^{2}}{2(2 \pi)^{D-1}} C_{\beta \alpha}^{-1} C_{\lambda \gamma} \int \frac{d^{D} k_{1}}{(2 \pi)^{D}} \int \frac{d^{D} k_{2}}{(2 \pi)^{D}} \\
& \times \cos ^{2}\left(\frac{k_{1} \wedge k_{2}}{2}\right) \widetilde{S}_{\beta \lambda}^{+}\left(k_{1}\right) \widetilde{S}_{\alpha \gamma}^{+}\left(k_{2}\right) \\
& \times\left[(2 \pi)^{D} \delta^{D}\left(-k_{1}-k_{2}-p_{2}\right)(2 \pi)^{D} \delta^{D}\left(k_{1}+k_{2}+p_{1}\right)\right. \\
& \left.+(2 \pi)^{D} \delta^{D}\left(-k_{1}-k_{2}+p_{1}\right)(2 \pi)^{D} \delta^{D}\left(k_{1}+k_{2}-p_{2}\right)\right] .
\end{aligned}
$$

Calculando a integral em $k_{2}$, finalmente obtemos o resultado

$$
\begin{aligned}
\left\langle p_{1}\left|A_{1}^{\dagger} A_{1}\right| p_{2}\right\rangle & =(2 \pi)^{D} \delta^{D}\left(p_{2}-p_{1}\right) \frac{\lambda^{2}}{2(2 \pi)^{D-1}} C_{\beta \alpha}^{-1} C_{\lambda \gamma} \int \frac{d^{D} k_{1}}{(2 \pi)^{D}} \\
& \times\left[\cos ^{2}\left(\frac{1}{2} k_{1} \wedge p_{2}\right) \widetilde{S}_{\beta \lambda}^{+}\left(k_{1}\right) \widetilde{S}_{\alpha \gamma}^{+}\left(-k_{1}-p_{2}\right)\right. \\
& \left.+\cos ^{2}\left(\frac{1}{2} k_{1} \wedge p_{1}\right) \widetilde{S}_{\beta \lambda}^{+}\left(k_{1}\right) \widetilde{S}_{\alpha \gamma}^{+}\left(-k_{1}+p_{1}\right)\right] .
\end{aligned}
$$




\section{Referências Bibliográficas}

[1] H.S. Snyder, Quantized Spacetime, Phys. Rev. 71 (1947) 38.

[2] N. Seiberg, E. Witten, String theory and noncommutative geometry, JHEP 9909 (1999) 032.

[3] S. Doplicher, K. Fredenhagen, J. B. Roberts, The Quantum Structure of the Spacetime at the Planck Scale and Quantum Fields, Commum. Math. Phys. 172 (1995) 187.

[4] S. Minwalla, M. V. Raamsdonk, N. Seiberg, Noncommutative perturbative dynamics, JHEP 02 (2000) 020, hep-th/9912072.

[5] J. Gomis, T. Mehen, Space-Time noncommutative field theories and unitarity, Nucl. Phys. B591, 265 (2000).

[6] R. J. Szabo, Quantum Field Theory on Noncommutative Spaces, Phys.Rept.378:207299, 2003, hep-th/0109162.

[7] M. R. Douglas, N. A. Nekrasov, Noncommutative field theory, Rev. Mod. Phys. 73, 977 - 1029 (2001), hep-th/0106048.

[8] Y. Liao, K. Sibold, Time-ordered perturbation theory on noncommutative spacetime I: Basic rules, Eur. Phys. J. C25, 469 (2002).

[9] Y. Liao, K. Sibold, Time-ordered perturbation theory on noncommutative spacetime II: Unitarity, Eur. Phys. J. C25, 479 (2002).

[10] T. Ohl, R. Ruckl, J. Zeiner, Unitarity of time-like noncommutative gauge theory: The Violation of Ward identities in time ordered perturbation theory, Nucl. Phys. B 676, 229 (2004), hep-th/0309021.

[11] D. Bahns, S. Doplicher, K. Fredenhagen, G. Piacitelli, On the unitarity problem in space-time noncommutative theories, Phys. Lett. B533, 178 (2002).

[12] C. Rim, J.H. Yee, Unitarity in space-time noncommutative field theory, Phys. Lett. B574, 111 (2003). 
[13] C. Rim, Y. Seo, J.H. Yee, Perturbation theory of the space-time noncommutative real scalar field theories, Phys. Rev. D70, 025006 (2004), hep-th/0312308.

[14] C. Rim, J.H. Yee, Scattering theory of space-time noncommutative gauge field theory, J.Korean Phys.Soc. 46 (2005) 781-790, hep-th/0401008.

[15] K. Fujikawa, Path Integral for Space-Time Noncommutative Field Theory, Phys. Rev. D 70, 085006 (2004), hep-th/0406128v1.

[16] A. Das, Finite Temperature Field Theory, World Scientific, Singapore, 1997.

[17] M. Veltman, Diagrammatica: The Path to Feynman Diagrams, Cambridge University Press, Cambridge, 1994.

[18] G. t'Hooft, M. Veltman, Diagrammar, CERN, Geneva, 1973.

[19] M. Gomes, Renormalization in Noncommutative Field Theory, XI Sumer School Jorge Andre Swieca. Section Particles and Fields, 2002.

[20] T. Filk, Divergences in a field theory on quantum space, Phys. Lett. B376, 53 (1996).

[21] M. Gomes, Teoria Quântica dos Campos, Editora da Universidade de São Paulo, São Paulo, 2002.

[22] C. Itzykson, J-B. Zuber, Quantum Field Theory, McGraw-Hill Book Co., 1980.

[23] I. S. Gradsteyn, I. M. Ryzhik, Table of Integrals, Series and Products, Academic Press, New York, 2000.

[24] K. Morita, Y. Okumura, E. Umezawa, Lorentz Invariance and Unitarity Problem in Noncommutative Field Theory, Prog.Theor.Phys. 109 (2003) 663-685, hepth/0309155v3. 